\title{
EXISTENCE OF SOLUTIONS OF SCALAR FIELD EQUATIONS WITH FRACTIONAL OPERATOR
}

\author{
NORIHISA IKOMA
}

\begin{abstract}
In this paper, the existence of least energy solution and infinitely many solutions is proved for the equation $(1-\Delta)^{\alpha} u=f(u)$ in $\mathbf{R}^{N}$ where $0<\alpha<1, N \geq 2$ and $f(s)$ is a Berestycki-Lions type nonlinearity. The characterization of the least energy by the mountain pass value is also considered and the existence of optimal path is shown. Finally, exploiting these results, the existence of positive solution for the equation $(1-\Delta)^{\alpha} u=f(x, u)$ in $\mathbf{R}^{N}$ is established under suitable conditions on $f(x, s)$.
\end{abstract}

\section{INTRODUCTION}

In this paper, we are concerned with the existence of nontrivial solutions of

$$
\left\{\begin{aligned}
(1-\Delta)^{\alpha} u & =f(x, u) \quad \text { in } \mathbf{R}^{N}, \\
u & \in H^{\alpha}\left(\mathbf{R}^{N}\right)
\end{aligned}\right.
$$

where $N \geq 2$ and $0<\alpha<1$. The fractional operator $(1-\Delta)^{\alpha} u$ is defined by

$$
(1-\Delta)^{\alpha} u:=\mathscr{F}^{-1}\left(\left(1+4 \pi^{2}|\xi|^{2}\right)^{\alpha} \widehat{u}(\xi)\right), \quad \hat{u}(\xi):=(\mathscr{F} u)(\xi)=\int_{\mathbf{R}^{N}} e^{-2 \pi x \cdot \xi} u(x) \mathrm{d} x
$$

and $H^{\alpha}\left(\mathbf{R}^{N}\right)$ a fractional Sobolev space consisted by real valued functions, that is,

$$
H^{\alpha}\left(\mathbf{R}^{N}\right):=\left\{\left.u \in L^{2}\left(\mathbf{R}^{N}, \mathbf{R}\right)\left|\|u\|_{\alpha}^{2}:=\int_{\mathbf{R}^{N}}\left(4 \pi^{2}|\xi|^{2}+1\right)^{\alpha}\right| \hat{u}\right|^{2} \mathrm{~d} \xi<\infty\right\} .
$$

Throughout this paper, we deal with a weak solution of (1), namely, a function $u \in H^{\alpha}\left(\mathbf{R}^{N}\right)$ satisfying

$$
\int_{\mathbf{R}^{N}}\left(4 \pi^{2}|\xi|^{2}+1\right)^{\alpha} \widehat{u}(\xi) \overline{\widehat{\varphi}(\xi)} \mathrm{d} \xi-\int_{\mathbf{R}^{N}} f(x, u(x)) \varphi(x) \mathrm{d} x=0 \quad \text { for all } \varphi \in H^{\alpha}\left(\mathbf{R}^{N}\right)
$$

where $\bar{a}$ denotes the complex conjugate of $a$.

The operator $(1-\Delta)^{\alpha}$ is related to the pseudo-relativistic Schrödinger operator $\left(m^{2}-\Delta\right)^{1 / 2}-m(m>0)$ and recently a lot of attentions are paid for equations involving them. Here we refer to $[2-4,12-17,21,23,32,34,38]$ and references therein for more details and physical context of $(1-\Delta)^{\alpha}$. In these papers, the authors study the existence of nontrivial solution and infinitely many solutions for the equations with $\left(m^{2}-\Delta\right)^{\alpha}$ and various nonlinearities.

This paper is especially motivated by two papers [23] and [34]. In [23], the existence of positive solution of (1) is proved under the following conditions on $f(x, s)$ :

(i) $f \in C\left(\mathbf{R}^{N} \times \mathbf{R}, \mathbf{R}\right)$.

(ii) For all $x \in \mathbf{R}^{N}, f(x, s) \geq 0$ if $s \geq 0$ and $f(x, s)=0$ if $s \leq 0$.

(iii) The function $s \mapsto s^{-1} f(x, s)$ is increasing in $(0, \infty)$ for all $x \in \mathbf{R}^{N}$.

(iv) There are $1<p<2_{\alpha}^{*}-1=(N+2 \alpha) /(N-2 \alpha)$ and $C>0$ such that $|f(x, s)| \leq C|s|^{p}$ for all $(x, s) \in \mathbf{R}^{N} \times \mathbf{R}$.

(v) There exists a $\mu>2$ such that $0<\mu F(x, s) \leq s f(x, s)$ for all $(x, s) \in \mathbf{R}^{N} \times(0, \infty)$ where $F(x, s):=$ $\int_{0}^{s} f(x, t) \mathrm{d} t$.

(vi) There exist continuous functions $\bar{f}(s)$ and $a(x)$ such that $\bar{f}$ satisfies (i)-(v) and $0 \leq f(x, s)-\bar{f}(s) \leq$ $a(x)\left(|s|+|s|^{p}\right)$ for all $(x, s) \in \mathbf{R}^{N} \times \mathbf{R}, a(x) \rightarrow 0$ as $|x| \rightarrow \infty$ and $\mathcal{L}^{N}\left(\left\{x \in \mathbf{R}^{N} \mid f(x, s)>\bar{f}(s)\right.\right.$ for all $\left.\left.s>0\right\}\right)>0$ where $\mathcal{L}^{N}$ denotes the $n$-dimensional Lebesgue measure.

On the other hand, in [34], the author obtains a nontrivial solution of (1) with $f(x, s)=\lambda b(x)|u|^{p-1} u+$ $c(x)|u|^{q-1} u$ under different conditions on $b(x), c(x), p, q$ where $\lambda>0$ is a constant. Among other things, under $1<p, q<2_{\alpha}^{*}-1$ and some strict inequality for the mountain pass value (the infimum of the functional on the

2010 Mathematics Subject Classification. 35J60, 35S05.

Key words and phrases. variational method, mountian pass theorem, symmetric mountain pass theorem, the Pohozaev identity. 
Nehari manifold), the existence of positive solution of (1) is shown. However, there is no specific information when the strict inequality holds.

Our aim of this paper is to observe whether we can handle the more general nonlinearity $f(x, s)$ in $(1)$ and obtain a positive solution. First, we treat the case where $f(x, s)=f(s)$ is a Berestycki-Lions type nonlinearity, that is, $f(s)$ satisfying (f1)-(f4) below. These conditions are introduced in [7,8] (cf. [6]) for the case $\alpha=1$ and almost optimal for the existence of nontrivial solution. We shall prove the existence of infinitely many solutions and least energy solutions with the Pohozaev identity, that the least energy coincides with the mountain pass value and that there is an optimal path. These properties are shown in $[24,26]$ for the case $\alpha=1$. Second, we deal with the case $f(x, s)$ depends on $x$. Here, exploiting the optimal path and characterization by the mountain pass value, we show the existence of positive solution of (1), which generalizes the result in [23] and enables us to find a simpler sufficient condition than that of [34] in some case. See the comments after Remark 1.4.

As stated in the above, we first consider the case $f(x, s) \equiv f(s)$ and $(1)$ becomes

$$
\left\{\begin{aligned}
(1-\Delta)^{\alpha} u & =f(u) \quad \text { in } \mathbf{R}^{N}, \\
u & \in H^{\alpha}\left(\mathbf{R}^{N}\right) .
\end{aligned}\right.
$$

For (4), we assume that the nonlinearity $f$ is a Berestycki-Lions type $([7,8])$ :

(f1) $f \in C(\mathbf{R}, \mathbf{R})$ and $f(s)$ is odd.

(f2) $-\infty<\liminf _{s \rightarrow 0} \frac{f(s)}{s} \leq \limsup _{s \rightarrow 0} \frac{f(s)}{s}<1$.

$$
\lim _{|s| \rightarrow \infty} \frac{|f(s)|}{|s|^{2_{\alpha}^{*}-1}}=0 \quad \text { where } 2_{\alpha}^{*}:=\frac{2 N}{N-2 \alpha} .
$$

(f4) There exists an $s_{0}>0$ such that

$$
F\left(s_{0}\right)-\frac{1}{2} s_{0}^{2}>0 \quad \text { where } F(s):=\int_{0}^{s} f(t) \mathrm{d} t .
$$

Notice that under (f1)-(f3), (4) has variational structure, namely, a solution of (4) is characterized as a critical point of the following functional (see Lemma 2.1)

$$
I(u):=\frac{1}{2}\|u\|_{\alpha}^{2}-\int_{\mathbf{R}^{N}} F(u) \mathrm{d} x \in C^{1}\left(H^{\alpha}\left(\mathbf{R}^{N}\right), \mathbf{R}\right) .
$$

Our first result is the existence of infinitely many solutions of (4) and the characterization of least energy solutions by the mountain pass structure.

Theorem 1.1. Assume $N \geq 2,0<\alpha<1$ and (f1)-(f4).

(i) There exist infinitely many solutions $\left(u_{n}\right)_{n=1}^{\infty}$ of (4) satisfying $I\left(u_{n}\right) \rightarrow \infty$ and the Pohozaev identity $P\left(u_{n}\right)=0$ where

$$
P(u):=\frac{N-2 \alpha}{2} \int_{\mathbf{R}^{N}}\left(1+4 \pi^{2}|\xi|^{2}\right)^{\alpha}|\widehat{u}|^{2} \mathrm{~d} \xi-N \int_{\mathbf{R}^{N}} F(u) \mathrm{d} x+\alpha \int_{\mathbf{R}^{N}}\left(1+4 \pi^{2}|\xi|^{2}\right)^{\alpha-1}|\widehat{u}|^{2} \mathrm{~d} \xi .
$$

Moreover, $u_{1}(x)>0$ for all $x \in \mathbf{R}^{N}$.

(ii) Assume either $\alpha>1 / 2$ or $f(s)$ is locally Lipschitz continuous. Then every solution of (4) satisfies the Pohozaev identity $P(u)=0$.

(iii) For the following quantities

$$
\begin{aligned}
c_{\mathrm{MP}} & :=\inf _{\gamma \in \Gamma} \max _{0 \leq t \leq 1} I(\gamma(t)), \quad \Gamma:=\left\{\gamma \in C\left([0,1], H^{\alpha}\left(\mathbf{R}^{N}\right)\right) \mid \gamma(0)=0, I(\gamma(1))<0\right\}, \\
c_{\mathrm{LES}} & :=\inf \left\{I(u) \mid u \not \equiv 0, I^{\prime}(u)=0, P(u)=0\right\}, \\
S_{\mathrm{LES}} & :=\left\{u \in H^{\alpha}\left(\mathbf{R}^{N}\right) \mid u \not \equiv 0, I^{\prime}(u)=0, P(u)=0, I(u)=c_{\mathrm{LES}}\right\},
\end{aligned}
$$

we have $S_{\mathrm{LES}} \neq \emptyset$ and $c_{\mathrm{MP}}=c_{\mathrm{LES}}>0$. Furthermore, for every $v \in S_{\mathrm{LES}}$, there exists a $\gamma_{v} \in \Gamma$ such that $\left\|\gamma_{v}(t)\right\|_{L^{\infty}}=\|v\|_{L^{\infty}}$ for $0<t \leq 1$ and

$$
\max _{0 \leq t \leq 1} I\left(\gamma_{v}(1)\right)=I(v)=c_{\text {LES }}
$$


Remark 1.2. (i) To the author's knowledge, it is not known that every weak solution of (4) satisfies the Pohozaev identity. In Proposition 3.6, we shall show that if a weak solution of (4) is of class $C^{1}$ with bounded derivatives, then the Pohozaev identity holds.

(ii) By Theorem 1.1 (ii), when $\alpha>1 / 2$ or $f(s)$ is locally Lipschitz, we have

$$
\begin{aligned}
c_{\mathrm{LES}} & =\inf \left\{I(u) \mid u \not \equiv 0, I^{\prime}(u)=0\right\}, \\
S_{\mathrm{LES}} & =\left\{u \in H^{\alpha}\left(\mathbf{R}^{N}\right) \mid u \not \equiv 0, I^{\prime}(u)=0, I(u)=c_{\mathrm{LES}}\right\} .
\end{aligned}
$$

Thus $c_{\mathrm{LES}}$ and $S_{\mathrm{LES}}$ coincide with the least energy and a set of all least energy solutions in usual sense.

(iii) By the simple scaling, we may deal with

$$
\left(m^{2}-\Delta\right)^{\alpha} u=f(u) \text { in } \mathbf{R}^{N}
$$

where $m>0$. Indeed, for $m>0, u(x)$ is a solution of (4) if and only if $v(x):=u\left(m^{-1} x\right)$ satisfies

$$
(1-\Delta)^{\alpha} v=m^{-2 \alpha} f(v(x)) \text { in } \mathbf{R}^{N} .
$$

Next, we use Theorem 1.1 to obtain a positive solution of (1). For $f(x, s)$, assume that

(F1) $f(x, s)=-V(x) s+g(x, s)$ where $V \in C\left(\mathbf{R}^{N}, \mathbf{R}\right), g \in C\left(\mathbf{R}^{N} \times \mathbf{R}, \mathbf{R}\right)$ and $g(x,-s)=-g(x, s)$ for every $(x, s) \in \mathbf{R}^{N} \times \mathbf{R}$.

$$
-1<\inf _{x \in \mathbf{R}^{N}} V(x) \text { and } \lim _{s \rightarrow 0} \sup _{x \in \mathbf{R}^{N}}\left|\frac{g(x, s)}{s}\right|=0 .
$$

$$
\lim _{|s| \rightarrow \infty} \sup _{x \in \mathbf{R}^{N}} \frac{|g(x, s)|}{|s|^{2_{\alpha}^{*}-1}}=0 .
$$

(F4) There exist $V_{\infty}>-1$ and $g_{\infty}(s) \in C(\mathbf{R}, \mathbf{R})$ such that as $|x| \rightarrow \infty, V(x) \rightarrow V_{\infty}$ and $g(x, s) \rightarrow g_{\infty}(s)$ in $L_{\text {loc }}^{\infty}\left(\mathbf{R}^{N}\right)$ where $g_{\infty}(s)$ is locally Lipschitz continuous provided $0<\alpha \leq 1 / 2$. Moreover, $0 \leq F(x, s)-$ $F_{\infty}(s)$ holds for all $x \in \mathbf{R}^{N}$ and $s \in \mathbf{R}$ where $F(x, s):=\int_{0}^{s} f(x, t) \mathrm{d} t, f_{\infty}(s):=-V_{\infty} s+g_{\infty}(s)$ and $F_{\infty}(s):=\int_{0}^{s} f_{\infty}(t) \mathrm{d} t$.

(F5) There exist $\mu>2$ and $s_{1}>0$ such that

$$
0<\mu G(x, s) \leq g(x, s) s \quad \text { for each }(x, s) \in \mathbf{R}^{N} \times \mathbf{R} \backslash\{0\}, \quad \inf _{x \in \mathbf{R}^{N}} G\left(x, s_{1}\right)>0
$$

where $G(x, s):=\int_{0}^{s} g(x, t) \mathrm{d} t$.

Under these conditions, we have

Theorem 1.3. Assume (F1)-(F5). Then (1) admits a positive solution.

Remark 1.4. (i) In (F4), when $0<\alpha \leq 1 / 2$, we assume that $g_{\infty}(s)$ is local Lipschitz in $s$, however, not for $g(x, s)$.

(ii) (F5) is mainly used to find a bounded Palais-Smale sequence. If we assume the existence of bounded Palais-Smale sequence at the mountain pass level, we can show the existence of nontrivial solution of (1) in the more general setting. See Proposition 4.3.

(iii) Another way to obtain bounded Palais-Smale sequences is to exploit the Pohozaev identity. When $\alpha=1$, for example, we refer to [5,28]. When $0<\alpha<1$, we also have the Pohozaev identity (see (46)) and it might be useful to get a bounded Palais-Smale sequence in the case $0<\alpha<1$.

Now we compare our result with the previous results. We first consider (4). The most related results are $[4,12,23,38]$. In these papers, the authors study (4) with $f(s)=|s|^{p-1} s$ or $f(s)=(1-\mu) s+|s|^{p-1} s$ where $1<p<2_{\alpha}^{*}-1$ and $\mu>0$, and show the existence of least energy solution (or ground state solution) and infinitely many solutions. Clearly, Theorem 1.1 improves these results. Furthermore, in [4,34], the authors raise a question that one can prove the existence of least energy solution and infinitely many solutions of (4) with general nonlinearity. Theorem 1.1 answers this question. For the fractional Laplacian $(-\Delta)^{\alpha}$ with general nonlinearity, we refer to the work [11].

On the other hand, for (1), the existence of positive solution is proved in [23,34]. It is easily checked that Theorem 1.3 is a generalization of the result in [23]. In addition, suppose that $f(x, s)=\lambda b(x)|u|^{p-1} u+c(x)|u|^{q-1} u$, $b(x) \geq \underline{b}=\lim _{|x| \rightarrow \infty} b(x)>0$ and $c(x) \geq 0=\lim _{|x| \rightarrow \infty} c(x)$. Then we can apply Theorem 1.3 to get a positive solution of (1) for every $\lambda>0$ and $1<p, q<2_{\alpha}^{*}-1$. Hence, in this case, we find the simpler sufficient condition than that of [34] for the existence of nontrivial solution 
Finally, we comment on the proofs of Theorems 1.1 and 1.3. Our arguments are variational and we find critical points of $I$ defined by (5) and

$$
J(u):=\frac{1}{2}\|u\|_{\alpha}^{2}-\int_{\mathbf{R}^{N}} F(x, u(x)) \mathrm{d} x \in C^{1}\left(H^{\alpha}\left(\mathbf{R}^{N}\right), \mathbf{R}\right) .
$$

To show the existence of critical points of $I$, we use the arguments in [24,25] and introduce the augmented functional based on the scaling:

$$
\tilde{I}(\theta, u):=I\left(u\left(\cdot / e^{\theta}\right)\right) \in C^{1}\left(\mathbf{R} \times H^{\alpha}\left(\mathbf{R}^{N}\right), \mathbf{R}\right) .
$$

As already pointed out in $[4,34],-\Delta$ and $(-\Delta)^{\alpha}$ are homogenous in scaling, however, $(1-\Delta)^{\alpha}$ is not. Nevertheless, $\tilde{I}$ still helps us to find bounded Palais-Smale sequences.

Next, we turn to Theorem 1.3. We use the idea of the concentration compactness lemma ( [27, 30,31]) and compare the mountain pass values. First, we treat the general setting and exploiting Theorem 1.1, we prove that it suffices to find a bounded Palais-Smale sequence of $J$ at the mountain pass level. To this end, we observe the behavior of any bounded Palais-Smale sequence of $J$. After that, we shall prove Theorem 1.3.

This paper is organized as follows. In sections 2 and 3 , we introduce the augmented functional $\tilde{I}(\theta, u)$, prove its properties and show Theorem 1.1. Section 4 is devoted to proving Theorem 1.3. In Appendix, we collect some technical lemmas and prove a Brézis-Kato type result (Proposition 3.5).

\section{VARIATONAL SETTING}

To prove Theorems 1.1 and 1.3, we employ the variational methods. We first consider (4) and prove Theorem 1.1. In what follows, we always assume (f1)-(f4) and use the following notations: For $K=\mathbf{R}, \mathbf{C}, \mathscr{S}\left(\mathbf{R}^{N}, K\right)$ denotes the Schwartz class consisting of $K$-valued functions. Moreover, set $H_{\mathrm{r}}^{\alpha}\left(\mathbf{R}^{N}\right):=\left\{u \in H^{\alpha}\left(\mathbf{R}^{N}\right) \mid u\right.$ is radial $\}$. Recalling (2), we begin with the following lemma.

Lemma 2.1. (i) The space $H^{\alpha}\left(\mathbf{R}^{N}\right)$ is a Hilbert space over $\mathbf{R}$ under the following scalar product:

$$
\langle u, v\rangle_{\alpha}:=\int_{\mathbf{R}^{N}}\left(4 \pi^{2}|\xi|^{2}+1\right)^{\alpha} \hat{u}(\xi) \overline{\hat{v}(\xi)} \mathrm{d} \xi .
$$

Notice that $\|u\|_{\alpha}^{2}=\langle u, u\rangle_{\alpha}$.

(ii) ( [29]) The embedding $H_{\mathrm{r}}^{\alpha}\left(\mathbf{R}^{N}\right) \subset L^{p}\left(\mathbf{R}^{N}\right)$ is compact for $2<p<2_{\alpha}^{*}$.

(iii) The functional $I$ in (5) belongs to $C^{1}\left(H^{\alpha}\left(\mathbf{R}^{N}\right), \mathbf{R}\right)$ and

$$
I^{\prime}(u) \varphi=\int_{\mathbf{R}^{N}}\left(1+4 \pi^{2}|\xi|^{2}\right)^{\alpha} \hat{u} \bar{\varphi} \mathrm{d} \xi-\int_{\mathbf{R}^{N}} f(u) \varphi \mathrm{d} x \quad \text { for all } \varphi \in H^{\alpha}\left(\mathbf{R}^{N}\right) .
$$

In particular, if $I^{\prime}(u)=0$, then $u$ satisfies (4) in $\left(\mathscr{S}\left(\mathbf{R}^{N}, \mathbf{C}\right)\right)^{*}$, that is, for all $\varphi \in \mathscr{S}\left(\mathbf{R}^{N}, \mathbf{C}\right)$,

$$
\left\langle(1-\Delta)^{\alpha} u, \varphi\right\rangle=\int_{\mathbf{R}^{N}}\left(1+4 \pi^{2}|\xi|^{2}\right)^{\alpha} \hat{u}(\xi)\left(\mathscr{F}^{-1} \varphi\right)(\xi) \mathrm{d} \xi=\int_{\mathbf{R}^{N}} f(u) \varphi \mathrm{d} x .
$$

The same holds true for $\left.I\right|_{H_{\mathrm{r}}^{\alpha}\left(\mathbf{R}^{N}\right)}$.

Proof. (i) We only check $\langle u, v\rangle_{\alpha} \in \mathbf{R}$ for any $u, v \in H^{\alpha}\left(\mathbf{R}^{N}\right)$. Put

$$
G_{2 \alpha}(x):=\frac{1}{(4 \pi)^{\alpha}} \frac{1}{\Gamma(\alpha)} \int_{0}^{\infty} e^{-\pi|x|^{2} / t} e^{-t / 4 \pi} t^{(2 \alpha-N) / 2} \frac{\mathrm{d} t}{t} .
$$

Then it is known that (see [35, Chapter V])

$$
\begin{aligned}
& \widehat{G_{2 \alpha}}(\xi)=\left(4 \pi^{2}|\xi|^{2}+1\right)^{-\alpha}, \quad\left\|G_{2 \alpha}\right\|_{L^{1}}=1, \\
& 0 \leq G_{2 \alpha}(x) \leq C_{0}\left(|x|^{N-2 \alpha} \chi_{B_{1}(0)}(x)+e^{-c_{1}|x|} \chi_{\left(B_{1}(0)\right)^{c}}(x)\right)
\end{aligned}
$$

for some $c_{1}>0$ where $B_{1}(0):=\left\{x \in \mathbf{R}^{N}|| x \mid<1\right\}$ and $\chi_{A}$ is a characteristic function of $A$. Moreover, for every $\varphi \in \mathscr{S}\left(\mathbf{R}^{N}, \mathbf{R}\right)$, the equation

$$
(-\Delta+1)^{\alpha} u=\varphi \quad \text { in } \mathbf{R}^{N}, \quad u \in H^{\alpha}\left(\mathbf{R}^{N}\right)
$$

has a unique solution $u$ expressed as $u=G_{2 \alpha} * \varphi \in \mathscr{S}\left(\mathbf{R}^{N}, \mathbf{R}\right)$ due to (9). For this $u$, if $v \in \mathscr{S}\left(\mathbf{R}^{N}, \mathbf{R}\right)$, then

$$
\begin{aligned}
\langle u, v\rangle_{\alpha} & =\int_{\mathbf{R}^{N}}\left(1+4 \pi|\xi|^{2}\right)^{\alpha} \hat{u}(\xi) \overline{\hat{v}(\xi)} \mathrm{d} \xi=\int_{\mathbf{R}^{N}}\left(1+4 \pi|\xi|^{2}\right)^{\alpha} \widehat{G_{2 \alpha} * \varphi} \overline{\hat{v}(\xi)} \mathrm{d} \xi \\
& =\int_{\mathbf{R}^{N}} \hat{\varphi} \overline{\hat{v}} \mathrm{~d} \xi=\int_{\mathbf{R}^{N}} \varphi v \mathrm{~d} x \in \mathbf{R} .
\end{aligned}
$$


Here at the last equality, we used the Plancherel theorem. Therefore, if $\varphi, v \in \mathscr{S}\left(\mathbf{R}^{N}, \mathbf{R}\right)$ and $u=G_{2 \alpha} * \varphi$, then $\langle u, v\rangle_{\alpha} \in \mathbf{R}$. Since $\mathscr{S}\left(\mathbf{R}^{N}, \mathbf{R}\right)$ is dense in $H^{\alpha}\left(\mathbf{R}^{N}\right)$, we have

$$
\left\langle G_{2 \alpha} * \varphi, v\right\rangle_{\alpha} \in \mathbf{R} \text { for all } \varphi \in \mathscr{S}\left(\mathbf{R}^{N}, \mathbf{R}\right), v \in H^{\alpha}\left(\mathbf{R}^{N}\right) .
$$

Finally, the map $\varphi \mapsto G_{2 \alpha} * \varphi: \mathscr{S}\left(\mathbf{R}^{N}, \mathbf{R}\right) \rightarrow \mathscr{S}\left(\mathbf{R}^{N}, \mathbf{R}\right)$ is bijective, by the density argument, we obtain $\langle u, v\rangle_{\alpha} \in \mathbf{R}$ for every $u, v \in H^{\alpha}\left(\mathbf{R}^{N}\right)$.

(ii) This is proved in [29].

(iii) Noting $\langle u, v\rangle_{\alpha} \in \mathbf{R}$ for all $u, v \in H^{\alpha}\left(\mathbf{R}^{N}\right)$ and (f1)-(f4), it is easy to check $I \in C^{1}\left(H^{\alpha}\left(\mathbf{R}^{N}\right), \mathbf{R}\right)$ and (7). For (8), we see from (7) that

$$
\int_{\mathbf{R}^{N}}\left(1+4 \pi^{2}|\xi|^{2}\right)^{\alpha} \hat{u} \overline{\hat{\psi}} \mathrm{d} \xi=\int_{\mathbf{R}^{N}} f(u) \bar{\psi} \mathrm{d} x \quad \text { for all } \psi \in \mathscr{S}\left(\mathbf{R}^{N}, \mathbf{C}\right) .
$$

Then setting $\varphi(x):=\overline{\psi(x)}$ and noting $\overline{\mathscr{F} \psi}=\mathscr{F}^{-1} \bar{\psi}=\mathscr{F}^{-1} \varphi$, one observes that (8) holds.

The last assertion follows from the principle of symmetric criticality. See [39].

Hereafter, we shall look for critical points of $\left.I\right|_{H_{\mathrm{r}}^{\alpha}\left(\mathbf{R}^{N}\right)}$. Following the arguments in [24], we first introduce a comparison functional $\bar{I}(u)$, which plays a role to show that the minimax values $c_{n}$ defined in (18) diverge as $n \rightarrow \infty$. To this end, we modify the nonlinearity $f(s)$. By (f2), choose $\delta_{0}>0$ and $s_{1}>0$ such that

$$
s f(s) \leq\left(1-2 \delta_{0}\right) s^{2} \quad \text { for all }|s| \leq s_{1} .
$$

Fixing a $p_{0} \in\left(1,2_{\alpha}^{*}-1\right)$, set

$$
h(s):=\left\{\begin{array}{ll}
\left(f(s)-\left(1-\delta_{0}\right) s\right)_{+}, & \text {if } s \geq 0, \\
-h(-s) & \text { if } s<0,
\end{array} \quad \bar{h}(s):= \begin{cases}s^{p_{0}} \sup _{0<t<s} \frac{h(t)}{t^{p_{0}}} & \text { if } s>0, \\
0 & \text { if } s=0, \\
-\bar{h}(-s) & \text { if } s<0\end{cases}\right.
$$

where $a_{+}:=\max \{0, a\}$. Finally, put $\bar{H}(s):=\int_{0}^{s} \bar{h}(t) \mathrm{d} t$. Then

Lemma 2.2. (i) $\bar{h} \in C(\mathbf{R})$ is odd, $\bar{h}(s) \geq 0$ for $s \geq 0, \bar{h} \not \equiv 0$ and $\bar{h}$ satisfies (f3).

(ii) There exists an $s_{2}>0$ such that $\bar{h}(s)=0=\bar{H}(s)$ for all $|s| \leq s_{2}$. In particular, there is a $C_{0}>0$ such that $|\bar{h}(s) s|+|\bar{H}(s)| \leq C_{0}|s|^{2}$ for each $s \in \mathbf{R}$.

(iii) $0 \leq\left(p_{0}+1\right) \bar{H}(s) \leq s \bar{h}(s)$ for any $s \in \mathbf{R}$.

(iv) $F(s)-\left(1-\delta_{0}\right) s^{2} / 2 \leq \bar{H}(s)$ for every $s \in \mathbf{R}$.

(v) Let $\left(u_{n}\right) \subset H_{\mathrm{r}}^{\alpha}\left(\mathbf{R}^{N}\right)$ satisfy $u_{n} \rightarrow u_{0}$ weakly in $H_{\mathrm{r}}^{\alpha}\left(\mathbf{R}^{N}\right)$ and $u_{n}(x) \rightarrow u_{0}(x)$ for a.e. $x \in \mathbf{R}^{N}$. Then

$$
\bar{H}\left(u_{n}\right) \rightarrow \bar{H}\left(u_{0}\right) \quad \text { strongly in } L^{1}\left(\mathbf{R}^{N}\right), \quad \bar{h}\left(u_{n}\right) \rightarrow \bar{h}\left(u_{0}\right) \quad \text { strongly in } L^{2 N /(N+2 \alpha)}\left(\mathbf{R}^{N}\right) .
$$

Proof. Since one can prove (i)-(iv) in a similar way to [24, Lemma 2.1 and Corollary 2.2], we omit the details. Now we shall prove $(\mathrm{v})$. Since both of the assertions can be proved in a similar way, we only $\operatorname{treat} \bar{h}\left(u_{n}\right) \rightarrow \bar{h}\left(u_{0}\right)$ strongly in $L^{2 N /(N+2 \alpha)}\left(\mathbf{R}^{N}\right)$. Noting that $\bar{h}$ satisfies (f3) thanks to the assertion (i), for each $\varepsilon>0$ there exists an $s_{\varepsilon}>0$ such that

$$
|\bar{h}(s)|^{2 N /(N+2 \alpha)} \leq \varepsilon|s|^{2_{\alpha}^{*}} \quad \text { if }|s| \geq s_{\varepsilon} .
$$

Set $\left[\left|u_{n}\right|<a\right]:=\left\{x \in \mathbf{R}^{N}|| u_{n}(x) \mid<a\right\}, \chi_{n, \varepsilon}(x):=\chi_{\left[\left|u_{n}\right|<s_{\varepsilon}\right]}(x)$ and $\chi_{0, \varepsilon}(x):=\chi_{\left[\left|u_{0}\right|<s_{\varepsilon}\right]}(x)$. Using $\bar{h}\left(u_{n}(x)\right)=\bar{h}\left(\chi_{n, \varepsilon}(x) u_{n}(x)\right)+\bar{h}\left(\left(1-\chi_{n, \varepsilon}(x)\right) u_{n}(x)\right)$ and writing $v_{n}(x):=\chi_{n, \varepsilon}(x) u_{n}(x), v_{0}(x):=\chi_{0, \varepsilon}(x) u_{0}(x)$, $w_{n}(x):=\left(1-\chi_{n, \varepsilon}(x)\right) u_{n}(x)$ and $w_{0}(x):=\left(1-\chi_{0, \varepsilon}(x)\right) u_{0}(x)$, we have

$$
\left|\bar{h}\left(u_{n}\right)-\bar{h}\left(u_{0}\right)\right| \leq\left|\bar{h}\left(\chi_{0, \varepsilon} v_{n}\right)-\bar{h}\left(v_{0}\right)\right|+\left|\bar{h}\left(\left(1-\chi_{0, \varepsilon}\right) v_{n}\right)\right|+\left|\bar{h}\left(w_{n}\right)-\bar{h}\left(w_{0}\right)\right| .
$$

Since $w_{n}(x) \neq 0$ implies $\left|w_{n}(x)\right| \geq s_{\varepsilon}$, it follows from (11) that

$$
\begin{aligned}
\sup _{n \geq 1} \int_{\mathbf{R}^{N}}\left|\bar{h}\left(w_{n}\right)-\bar{h}\left(w_{0}\right)\right|^{2 N /(N+2 \alpha)} \mathrm{d} x & \leq C_{0} \sup _{n \geq 1} \int_{\mathbf{R}^{N}}\left(\left|\bar{h}\left(w_{n}\right)\right|^{2 N /(N+2 \alpha)}+\left|\bar{h}\left(w_{0}\right)\right|^{2 N /(N+2 \alpha)}\right) \mathrm{d} x \\
& \leq C \varepsilon \sup _{n \geq 1}\left(\left\|w_{n}\right\|_{L_{\alpha}^{2 *}}^{2^{*}}+\left\|w_{0}\right\|_{L^{2} 2_{\alpha}^{*}}^{2_{\alpha}^{*}}\right) \leq C \varepsilon
\end{aligned}
$$

Recalling $u_{n}(x) \rightarrow u_{0}(x)$ and the definition of $\chi_{0, \varepsilon}(x)$, we observe that

$$
\limsup _{n \rightarrow \infty}\left|\left(1-\chi_{0, \varepsilon}\right)(x) v_{n}(x)\right| \leq \chi_{\left[\left|u_{0}\right|=s_{\varepsilon}\right]}(x)\left|s_{\varepsilon}\right| \quad \text { for a.e. } x \in \mathbf{R}^{N} \text {. }
$$


Hence, using (11) and $\chi_{\left[\left|u_{0}\right|=s_{\varepsilon}\right]}(x) \leq\left(1-\chi_{0, \varepsilon}(x)\right)$, we obtain

$$
\begin{aligned}
\limsup _{n \rightarrow \infty} \int_{\mathbf{R}^{N}}\left|\bar{h}\left(\left(1-\chi_{0, \varepsilon}\right)(x) v_{n}(x)\right)\right|^{2 N /(N+2 \alpha)} \mathrm{d} x & \leq \int_{\mathbf{R}^{N}}\left|\bar{h}\left(\chi_{\left[\left|u_{0}\right|=s_{\varepsilon}\right]}(x)\left|s_{\varepsilon}\right|\right)\right|^{2 N /(N+2 \alpha)} \mathrm{d} x \\
& \leq \int_{\mathbf{R}^{N}}\left|\bar{h}\left(w_{0}\right)\right|^{2 N /(N+2 \alpha)} \mathrm{d} x \leq C \varepsilon .
\end{aligned}
$$

On the other hand, since $\chi_{0, \varepsilon}(x) v_{n}(x) \rightarrow v_{0}(x)$ for a.e. $x \in \mathbf{R}^{N}$, noting that $u_{n} \rightarrow u_{0}$ strongly in $L^{p}\left(\mathbf{R}^{N}\right)$ for $2<p<2_{\alpha}^{*}$ due to Lemma 2.1 (ii) and that $\left|v_{n}(x)\right|,\left|v_{0}(x)\right| \leq s_{\varepsilon}$, we have $\chi_{0, \varepsilon} v_{n} \rightarrow v_{0}$ strongly in $L^{p}\left(\mathbf{R}^{N}\right)$ for $2<p<\infty$. Thus, by the assertion (ii), it is easily seen that

$$
\lim _{n \rightarrow \infty} \int_{\mathbf{R}^{N}}\left|\bar{h}\left(\chi_{0, \varepsilon} v_{n}\right)-\bar{h}\left(v_{0}\right)\right|^{2 N /(N+2 \alpha)} \mathrm{d} x=0 .
$$

Collecting (12)-(15), one sees

$$
\limsup _{n \rightarrow \infty}\left\|\bar{h}\left(u_{n}\right)-\bar{h}\left(u_{0}\right)\right\|_{L^{2 N /(N+2 \alpha)}}^{2 N /(N+2 \alpha)} \leq C \varepsilon
$$

Since $\varepsilon>0$ is arbitrary, $\bar{h}\left(u_{n}\right) \rightarrow \bar{h}\left(u_{0}\right)$ strongly in $L^{2 N /(N+2 \alpha)}\left(\mathbf{R}^{N}\right)$.

Next, from

$$
\begin{aligned}
I(u) & =\frac{1}{2}\|u\|_{\alpha}^{2}-\frac{1-\delta_{0}}{2} \int_{\mathbf{R}^{N}} u^{2} \mathrm{~d} x-\int_{\mathbf{R}^{N}} F(u)-\frac{1-\delta_{0}}{2} u^{2} \mathrm{~d} x \\
& \geq \frac{\delta_{0}}{2}\|u\|_{\alpha}^{2}-\int_{\mathbf{R}^{N}} F(u)-\frac{1-\delta_{0}}{2} u^{2} \mathrm{~d} x,
\end{aligned}
$$

we define a comparison functional $\bar{I}(u)$ by

$$
\bar{I}(u):=\frac{\delta_{0}}{2}\|u\|_{\alpha}^{2}-\int_{\mathbf{R}^{N}} \bar{H}(u) \mathrm{d} x .
$$

Lemma 2.3. (i) The inequality $\bar{I}(u) \leq I(u)$ holds for any $u \in H^{\alpha}\left(\mathbf{R}^{N}\right)$. Moreover, there exists a $\rho_{0}>0$ such that

$$
0<\inf _{\|u\|_{\alpha}=\rho_{0}} \bar{I}(u), \quad \bar{I}(u) \geq 0 \quad \text { if }\|u\|_{\alpha} \leq \rho_{0} .
$$

(ii) The functional $\bar{I}$ satisfies the Palais-Smale condition.

(iii) For each $n \geq 1$, there exists a $\gamma_{n} \in C\left(\partial D_{n}, H_{\mathrm{r}}^{\alpha}\left(\mathbf{R}^{N}\right)\right)$ such that

$$
\gamma_{n}(-\sigma)=-\gamma_{n}(\sigma), \quad I\left(\gamma_{n}(\sigma)\right)<0 \quad \text { for each } \sigma \in \partial D_{n}
$$

where $D_{n}:=\left\{\sigma=\left(\sigma_{1}, \ldots, \sigma_{n}\right) \in \mathbf{R}^{n}|| \sigma \mid \leq 1\right\}$.

Proof. (i) The inequality $\bar{I}(u) \leq I(u)$ is clear from the definition and Lemma 2.2. Next by Lemma 2.2 (ii), we have $|\bar{H}(s)| \leq C|s|^{2_{\alpha}^{*}}$ for all $s \in \mathbf{R}$. Thus it follows from Sobolev's inequality that

$$
\bar{I}(u) \geq \frac{\delta_{0}}{2}\|u\|_{\alpha}^{2}-C \int_{\mathbf{R}^{N}}|u|^{2_{\alpha}^{*}} \mathrm{~d} x \geq \frac{\delta_{0}}{2}\|u\|_{\alpha}^{2}-C\|u\|_{\alpha}^{2_{\alpha}^{*}} .
$$

Noting $2<2_{\alpha}^{*}$ and choosing $\rho_{0}>0$ sufficiently small, we get

$$
\inf _{\|u\|_{\alpha}=\rho_{0}} I(u)>0, \quad I(u) \geq 0 \quad \text { if }\|u\|_{\alpha} \leq \rho_{0} .
$$

(ii) Since the nonlinearity $\bar{h}$ satisfies the global Ambrosetti-Rabinowitz condition (Lemma 2.2 (iii)), following the argument in [33] (cf. proof of Theorem 1.3 below) and using Lemma 2.2 (v), we can prove that $\bar{I}$ satisfies the Palais-Smale condition and we omit the details.

(iii) Since $f(s)-s$ satisfies the Berestycki-Lions type conditions (see $[7,8]$ ), as in [8, Theorem 10], we may find a map $\pi_{n} \in C\left(\partial D_{n}, H_{\mathrm{r}}^{1}\left(\mathbf{R}^{N}\right)\right)$ with the properties

$$
0 \notin \pi_{n}\left(\partial D_{n}\right), \quad \pi_{n}(-\sigma)=-\pi_{n}(\sigma), \quad \int_{\mathbf{R}^{N}} F\left(\pi_{n}(\sigma)\right)-\frac{1}{2}\left(\pi_{n}(\sigma)\right)^{2} \mathrm{~d} x \geq 1 \quad \text { for all } \sigma \in \partial D_{n} .
$$


Set $\gamma_{n}(\sigma)(x):=\pi_{n}(\sigma)(x / t)$ for $t>0$. Then for sufficiently large $t>0$, it follows from $\widehat{\gamma_{n}(\sigma)}(\xi)=t^{N} \widehat{\pi_{n}(\sigma)}(t \xi)$ and the inequality $(1+s)^{\alpha} \leq 1+s^{\alpha}$ for $s \geq 0$ that

$$
\begin{aligned}
I\left(\gamma_{n}(\sigma)\right) & =\left.\frac{t^{N}}{2} \int_{\mathbf{R}^{N}}\left(1+\frac{4 \pi^{2}|\xi|^{2}}{t^{2}}\right)^{\alpha} \widehat{\mid \pi_{n}(\sigma)}\right|^{2} \mathrm{~d} \xi-t^{N} \int_{\mathbf{R}^{N}} F\left(\pi_{n}(\sigma)\right) \mathrm{d} x \\
& \leq \frac{t^{N-2 \alpha}}{2} \int_{\mathbf{R}^{N}}\left(4 \pi^{2}|\xi|^{2}\right)^{\alpha}\left|\widehat{\pi_{n}(\sigma)}\right|^{2} \mathrm{~d} \xi-t^{N} \int_{\mathbf{R}^{N}} F\left(\pi_{n}(\sigma)\right)-\frac{1}{2}\left(\pi_{n}(\sigma)\right)^{2} \mathrm{~d} x \\
& \leq \frac{t^{N-2 \alpha}}{2} \int_{\mathbf{R}^{N}}\left(4 \pi^{2}|\xi|^{2}\right)^{\alpha}\left|\widehat{\pi_{n}(\sigma)}\right|^{2} \mathrm{~d} \xi-t^{N}<0 \quad \text { for all } \sigma \in \partial D_{n} .
\end{aligned}
$$

Since $H_{\mathrm{r}}^{1}\left(\mathbf{R}^{N}\right) \subset H_{\mathrm{r}}^{\alpha}\left(\mathbf{R}^{N}\right)$, we have $\gamma_{n} \in C\left(\partial D_{n}, H_{\mathrm{r}}^{\alpha}\left(\mathbf{R}^{N}\right)\right)$ and complete the proof.

Remark 2.4. When $n=1$, we can assume that $\gamma_{1}(1)(x) \geq 0$ for each $x \in \mathbf{R}^{N}, \gamma_{1}(1)(|x|)=\gamma_{1}(1)(x)$ and $r \mapsto \gamma_{1}(1)(r)$ is piecewise linear and nonincreasing. See [7,8] (cf. the proof of Proposition 4.1 below).

Now we introduce an auxiliary functional based on the scaling property as in [24,25]. For this purpose, we set

$$
u_{\theta}(x):=u\left(e^{-\theta} x\right) .
$$

Then we have

$$
\widehat{u_{\theta}}(\xi)=e^{N \theta} \hat{u}\left(e^{\theta} \xi\right), \quad I\left(u_{\theta}\right)=\frac{e^{N \theta}}{2} \int_{\mathbf{R}^{N}}\left(1+4 \pi^{2} \frac{|\xi|^{2}}{e^{2 \theta}}\right)^{\alpha}|\hat{u}|^{2} \mathrm{~d} \xi-e^{N \theta} \int_{\mathbf{R}^{N}} F(u) \mathrm{d} x .
$$

From this, we define $\tilde{I}(\theta, u)$ by

$$
\tilde{I}(\theta, u):=\frac{e^{N \theta}}{2} \int_{\mathbf{R}^{N}}\left(1+4 \pi^{2} \frac{|\xi|^{2}}{e^{2 \theta}}\right)^{\alpha}|\hat{u}|^{2} \mathrm{~d} \xi-e^{N \theta} \int_{\mathbf{R}^{N}} F(u) \mathrm{d} x .
$$

It is easily seen that $\tilde{I} \in C^{1}\left(\mathbf{R} \times H_{\mathrm{r}}^{\alpha}\left(\mathbf{R}^{N}\right), \mathbf{R}\right)$ and $u$ is a critical point of $I$ if $(0, u)$ is a critical point of $\tilde{I}$. Furthermore, we see the following relation between $\tilde{I}$ and $P$ (see (6) for the definition of $P(u)$ ): for all $u \in H^{\alpha}\left(\mathbf{R}^{N}\right)$ and $\theta \in \mathbf{R}$,

$$
\begin{aligned}
D_{\theta} \tilde{I}(\theta, u)= & \frac{N}{2} e^{N \theta} \int_{\mathbf{R}^{N}}\left(1+4 \pi^{2} e^{-2 \theta}|\xi|^{2}\right)^{\alpha}|\hat{u}(\xi)|^{2} \mathrm{~d} \xi \\
& \quad-\alpha e^{N \theta} \int_{\mathbf{R}^{N}}\left(1+4 \pi^{2} e^{-2 \theta}|\xi|^{2}\right)^{\alpha-1} e^{-2 \theta} 4 \pi^{2}|\xi|^{2}|\hat{u}(\xi)|^{2} \mathrm{~d} \xi-N e^{N \theta} \int_{\mathbf{R}^{N}} F(u) \mathrm{d} x \\
= & \frac{N}{2} \int_{\mathbf{R}^{N}}\left(1+4 \pi^{2}|\xi|^{2}\right)^{\alpha}\left|\widehat{u_{\theta}}(\xi)\right|^{2} \mathrm{~d} \xi \\
& \quad-\alpha \int_{\mathbf{R}^{N}}\left(1+4 \pi^{2}|\xi|^{2}\right)^{\alpha-1} 4 \pi^{2}|\xi|^{2}\left|\widehat{u_{\theta}}(\xi)\right|^{2} \mathrm{~d} \xi-N \int_{\mathbf{R}^{N}} F(u) \mathrm{d} x \\
= & D_{\theta} \tilde{I}\left(0, u_{\theta}\right)=\frac{N-2 \alpha}{2}\left\|u_{\theta}\right\|_{\alpha}^{2}+\alpha \int_{\mathbf{R}^{N}}\left(1+4 \pi^{2}|\xi|^{2}\right)^{\alpha-1}\left|\widehat{u_{\theta}}\right|^{2} \mathrm{~d} \xi-N \int_{\mathbf{R}^{N}} F\left(u_{\theta}\right) \mathrm{d} x \\
= & P\left(u_{\theta}\right) .
\end{aligned}
$$

This functional is useful to generate a bounded Palais-Smale sequence $\left(u_{k}\right)$ with $P\left(u_{k}\right) \rightarrow 0$.

Recalling Lemma 2.3, for every $n \geq 1$, we define

$$
\begin{aligned}
& c_{n}:=\inf _{\gamma \in \Gamma_{n}} \max _{\sigma \in D_{n}} I(\gamma(\sigma)), \quad \tilde{c}_{n}:=\inf _{\tilde{\gamma} \in \tilde{\Gamma}_{n}} \max _{\sigma \in D_{n}} \tilde{I}(\tilde{\gamma}(\sigma)), \quad d_{n}:=\inf _{\gamma \in \Gamma_{n}} \max _{\sigma \in D_{n}} \bar{I}(\gamma(\sigma)), \\
& \Gamma_{n}:=\left\{\gamma \in C\left(D_{n}, H_{\mathrm{r}}^{\alpha}\left(\mathbf{R}^{N}\right)\right) \mid \gamma(-\sigma)=-\gamma(\sigma), \gamma=\gamma_{n} \text { on } \partial D_{n}\right\}, \\
& \tilde{\Gamma}_{n}:=\left\{\tilde{\gamma}(\sigma)=(\theta(\sigma), \gamma(\sigma)) \in C\left(D_{n}, \mathbf{R} \times H_{\mathrm{r}}^{\alpha}\left(\mathbf{R}^{N}\right)\right) \mid\right. \\
&\left.\quad \theta(-\sigma)=\theta(\sigma) \text { for all } \sigma \in D_{n}, \theta(\sigma)=0 \text { on } \partial D_{n}, \gamma \in \Gamma_{n}\right\}
\end{aligned}
$$

Remark that $\Gamma_{n} \neq \emptyset$ since $\gamma_{n, 0} \in \Gamma_{n}$ where $\gamma_{n, 0}(\sigma):=|\sigma| \gamma_{n}(\sigma /|\sigma|)$ when $\sigma \in D_{n} \backslash\{0\}$ and $\gamma_{n, 0}(0):=0$. Furthermore, we have

Lemma 2.5. For all $n \in \mathbf{N}, d_{n} \leq c_{n}=\tilde{c}_{n}$ hold and $d_{n} \rightarrow \infty$ as $n \rightarrow \infty$.

Proof. By definition and Lemma 2.3, $d_{n} \leq c_{n}$ is clear. Moreover, noting $(0, \gamma) \in \tilde{\Gamma}_{n}$ for all $\gamma \in \Gamma_{n}$ and $I(u)=$ $\tilde{I}(0, u)$, we have $\tilde{c}_{n} \leq c_{n}$ for all $n \in \mathbf{N}$. On the other hand, let $\tilde{\gamma}=(\theta, \gamma) \in \tilde{\Gamma}_{n}$ and put $\zeta(\sigma):=\gamma(\sigma)\left(e^{-\theta(\sigma)} x\right)$, it follows from $(16)$ that $I(\zeta(\sigma))=\tilde{I}(\theta(\sigma), \gamma(\sigma))$. From this and $\tilde{\gamma} \in \tilde{\Gamma}$, one can check that $\zeta \in \Gamma_{n}$ and $c_{n} \leq \tilde{c}_{n}$. Thus $c_{n}=\tilde{c}_{n}$ holds. 
The assertion $d_{n} \rightarrow \infty$ can be proved in a similar way to [24, Lemma 3.2] (see also [33]) and we skip the details.

\section{Proof of Theorem 1.1}

In this section, we shall prove Theorem 1.1. We first show the assertion (i). To proceed, we first notice that

$$
\begin{aligned}
D_{u} \tilde{I}(\theta, u) \varphi & =e^{N \theta} \int_{\mathbf{R}^{N}}\left(1+4 \pi^{2} e^{-2 \theta}|\xi|^{2}\right)^{\alpha} \hat{u} \bar{\varphi} \mathrm{d} \xi-e^{N \theta} \int_{\mathbf{R}^{N}} f(u) \varphi \mathrm{d} x \\
& =\int_{\mathbf{R}^{N}}\left(1+4 \pi^{2}|\xi|^{2}\right)^{\alpha} \widehat{u_{\theta}} \widehat{\widehat{\varphi_{\theta}}} \mathrm{d} \xi-\int_{\mathbf{R}^{N}} f\left(u_{\theta}\right) \varphi_{\theta} \mathrm{d} x=D_{u} \tilde{I}\left(0, u_{\theta}\right) \varphi_{\theta}
\end{aligned}
$$

where $\varphi_{\theta}(x):=\varphi\left(e^{-\theta} x\right)$.

Proposition 3.1. Suppose that $\left(\left(\theta_{n}, u_{n}\right)\right)_{n=1}^{\infty} \subset \mathbf{R} \times H_{\mathrm{r}}^{\alpha}\left(\mathbf{R}^{N}\right)$ is a Palais-Smale sequence of $\tilde{I}$, namely, $\tilde{I}\left(\theta_{n}, u_{n}\right) \rightarrow$ $c \in \mathbf{R}$ and $D_{(\theta, u)} \tilde{I}\left(\theta_{n}, u_{n}\right) \rightarrow 0$ strongly in $\mathbf{R} \times\left(H_{\mathrm{r}}^{\alpha}\left(\mathbf{R}^{N}\right)\right)^{*}$. Moreover, assume that $\left(\theta_{n}\right)$ is bounded. Then $\left(u_{n}\right)$ is bounded in $H_{\mathrm{r}}^{\alpha}\left(\mathbf{R}^{N}\right)$.

Proof. Since $\left(\theta_{n}\right)$ is bounded, from (17) and (19), we may assume $\theta_{n}=0$ by replacing $u_{n}(x)$ by $u_{n}\left(e^{-\theta_{n}} x\right)$. Therefore, we have

$$
\begin{aligned}
& c+o(1)=\tilde{I}\left(0, u_{n}\right)=\frac{1}{2}\left\|u_{n}\right\|_{\alpha}^{2}-\int_{\mathbf{R}^{N}} F\left(u_{n}\right) \mathrm{d} x, \\
& o(1)=D_{\theta} \tilde{I}\left(0, u_{n}\right)=\frac{N}{2}\left\|u_{n}\right\|_{\alpha}^{2}-N \int_{\mathbf{R}^{N}} F\left(u_{n}\right) \mathrm{d} x-4 \pi^{2} \alpha \int_{\mathbf{R}^{N}}\left(1+4 \pi^{2}|\xi|^{2}\right)^{\alpha-1}|\xi|^{2}\left|\widehat{u_{n}}\right|^{2} \mathrm{~d} \xi .
\end{aligned}
$$

From these, it follows that

$$
\left(\int_{\mathbf{R}^{N}}\left(1+4 \pi^{2}|\xi|^{2}\right)^{\alpha-1}|\xi|^{2}\left|\widehat{u_{n}}\right|^{2} \mathrm{~d} \xi\right)_{n=1}^{\infty} \text { is bounded. }
$$

Thus if

$$
\left(\left\|u_{n}\right\|_{L^{2}}^{2}\right)_{n=1}^{\infty}=\left(\int_{\mathbf{R}^{N}}\left|\widehat{u_{n}}\right|^{2} \mathrm{~d} \xi\right)_{n=1}^{\infty} \text { is bounded, }
$$

then from $\left(1+4 \pi^{2}|\xi|^{2}\right)^{\alpha-1} \leq 1$ and (20) we get

$$
\begin{aligned}
\left\|u_{n}\right\|_{\alpha}^{2} & =\int_{\mathbf{R}^{N}}\left(1+4 \pi^{2}|\xi|^{2}\right)^{\alpha-1}\left(1+4 \pi^{2}|\xi|^{2}\right)\left|\widehat{u_{n}}(\xi)\right|^{2} \mathrm{~d} \xi \\
& \leq \int_{\mathbf{R}^{N}}\left|\widehat{u_{n}}\right|^{2} \mathrm{~d} \xi+4 \pi^{2} \int_{\mathbf{R}^{N}}\left(1+4 \pi^{2}|\xi|^{2}\right)^{\alpha-1}|\xi|^{2}\left|\widehat{u_{n}}\right|^{2} \mathrm{~d} \xi
\end{aligned}
$$

and $\left(u_{n}\right)$ is bounded in $H^{\alpha}\left(\mathbf{R}^{N}\right)$.

Now we prove (21) by contradiction and suppose that $\tau_{n}:=\left\|u_{n}\right\|_{L^{2}}^{-2 / N} \rightarrow 0$ as $n \rightarrow \infty$. Set $v_{n}(x):=u_{n}\left(\tau_{n}^{-1} x\right)$ and observe that

$$
\left\|v_{n}\right\|_{L^{2}}^{2}=1, \quad \int_{\mathbf{R}^{N}}|\xi|^{2 \alpha}\left|\widehat{v_{n}}\right|^{2} \mathrm{~d} \xi=\tau_{n}^{N-2 \alpha} \int_{\mathbf{R}^{N}}|\xi|^{2 \alpha}\left|\widehat{u_{n}}\right|^{2} \mathrm{~d} \xi
$$

Since there exist $C_{1}, C_{2}>0$ such that

$$
C_{1}|\xi|^{2} \leq\left(1+4 \pi^{2}|\xi|^{2}\right)^{\alpha-1}|\xi|^{2} \quad \text { if }|\xi| \leq 1, \quad C_{2}\left(1+4 \pi^{2}|\xi|^{2}\right)^{\alpha} \leq\left(1+4 \pi^{2}|\xi|^{2}\right)^{\alpha-1}|\xi|^{2} \quad \text { if }|\xi| \geq 1,
$$

we observe from (20) that the quantities

$$
\left(\int_{|\xi| \geq 1}\left(1+4 \pi^{2}|\xi|^{2}\right)^{\alpha}\left|\widehat{u_{n}}\right|^{2} \mathrm{~d} \xi\right)_{n=1}^{\infty} \text { and }\left(\int_{|\xi| \leq 1}|\xi|^{2}\left|\widehat{u_{n}}\right|^{2} \mathrm{~d} \xi\right)_{n=1}^{\infty} \text { are bounded. }
$$

Thus we infer that

$$
\int_{|\xi| \leq 1}\left|\widehat{u_{n}}\right|^{2} \mathrm{~d} \xi \rightarrow \infty
$$

Now we divide our arguments into three steps. First we show

Step 1: When $N \geq 3, v_{n} \rightarrow 0$ weakly in $H_{\mathrm{r}}^{\alpha}\left(\mathbf{R}^{N}\right)$. 
We first see that for $q>1$, by Hölder's inequality and (23), we obtain

$$
\begin{aligned}
\int_{|\xi| \leq 1}|\xi|^{2 \alpha}\left|\widehat{u_{n}}\right|^{2} \mathrm{~d} \xi & \leq\left(\int_{|\xi| \leq 1}|\xi|^{2}\left|\widehat{u_{n}}\right|^{2} \mathrm{~d} \xi\right)^{1 / q}\left(\int_{|\xi| \leq 1}|\xi|^{2(\alpha q-1) /(q-1)}\left|\widehat{u_{n}}\right|^{2} \mathrm{~d} \xi\right)^{1-1 / q} \\
& \leq C_{q}\left(\int_{|\xi| \leq 1}|\xi|^{2(\alpha q-1) /(q-1)}\left|\widehat{u_{n}}\right|^{2} \mathrm{~d} \xi\right)^{1-1 / q}
\end{aligned}
$$

Choosing $q=\alpha^{-1} \in(1, \infty)$, one gets

$$
\int_{|\xi| \leq 1}|\xi|^{2 \alpha}\left|\widehat{u_{n}}\right|^{2} \mathrm{~d} \xi \leq C_{\alpha}\left(\int_{|\xi| \leq 1}\left|\widehat{u_{n}}\right|^{2} \mathrm{~d} \xi\right)^{1-\alpha} \leq C_{\alpha} \tau_{n}^{-N(1-\alpha)}
$$

Thus it follows from (22), (23) and $N \geq 3>2 \alpha$ that

$$
\begin{aligned}
\int_{\mathbf{R}^{N}}|\xi|^{2 \alpha}\left|\widehat{v_{n}}\right|^{2} \mathrm{~d} \xi & =\tau_{n}^{N-2 \alpha}\left(\int_{|\xi| \leq 1}+\int_{|\xi| \geq 1}\right)|\xi|^{2 \alpha}\left|\widehat{u_{n}}\right|^{2} \mathrm{~d} \xi \\
& \leq C_{\alpha} \tau_{n}^{(N-2) \alpha}+\tau_{n}^{N-2 \alpha} \int_{|\xi| \geq 1}|\xi|^{2 \alpha}\left|\widehat{u_{n}}\right|^{2} \mathrm{~d} \xi \rightarrow 0 .
\end{aligned}
$$

By Sobolev's inequality $\|u\|_{L^{2 *}} \leq C\left\||\xi|^{\alpha} \widehat{u}\right\|_{L^{2}}$, we obtain $v_{n} \rightarrow 0$ strongly in $L^{2_{\alpha}^{*}}\left(\mathbf{R}^{N}\right)$. Thus $v_{n} \rightarrow 0$ weakly in $H_{\mathrm{r}}^{\alpha}\left(\mathbf{R}^{N}\right)$.

Step 2: $v_{n} \rightarrow 0$ weakly in $H_{\mathrm{r}}^{\alpha}\left(\mathbf{R}^{N}\right)$ when $N=2$.

Let $\zeta_{0} \in C_{0}^{\infty}\left(\mathbf{R}^{N}\right)$ satisfy $0 \leq \zeta_{0} \leq 1, \zeta_{0}(\xi)=1$ for $|\xi| \leq 1$ and $\zeta_{0}(\xi)=0$ for $|\xi| \geq 2$. Set

$$
\zeta_{n}(\xi):=\zeta_{0}\left(\tau_{n} \xi\right), \quad w_{n, 1}(x):=\mathscr{F}^{-1}\left(\zeta_{n}(\xi) \widehat{v_{n}}(\xi)\right), \quad w_{n, 2}(x):=\mathscr{F}^{-1}\left(\left(1-\zeta_{n}(\xi)\right) \widehat{v_{n}}(\xi)\right) .
$$

Then one sees from the Plancherel theorem and (23) that $v_{n}=w_{n, 1}+w_{n, 2}$ and

$$
\begin{aligned}
& \left\|w_{n, 1}\right\|_{L^{2}}^{2}=\left\|\widehat{w_{n, 1}}\right\|_{L^{2}}^{2}=\int_{\mathbf{R}^{2}} \zeta_{n}^{2}\left|\widehat{v_{n}}\right|^{2} \mathrm{~d} \xi \leq\left\|v_{n}\right\|_{L^{2}}^{2} \leq 1, \\
& \left\|w_{n, 2}\right\|_{L^{2}}^{2}=\tau_{n}^{2} \int_{|\xi| \geq 1}\left(1-\zeta_{0}(\xi)\right)^{2}\left|\widehat{u_{n}}\right|^{2} \mathrm{~d} \xi \rightarrow 0, \\
& \int_{\mathbf{R}^{N}} w_{n, 1} \overline{w_{n, 2}} \mathrm{~d} x=\int_{\mathbf{R}^{N}} \widehat{w_{n, 1}} \overline{\widehat{w_{n, 2}}} \mathrm{~d} \xi=\tau_{n}^{2} \int_{1 \leq|\xi| \leq 2} \zeta_{0}(\xi)\left(1-\zeta_{0}(\xi)\right)\left|\widehat{u_{n}}\right|^{2} \mathrm{~d} \xi \rightarrow 0 .
\end{aligned}
$$

We also see that

$$
\int_{\mathbf{R}^{2}}|\xi|^{2}\left|\widehat{w_{n, 1}}\right|^{2} \mathrm{~d} \xi=\tau_{n}^{4} \int_{\mathbf{R}^{2}}|\xi|^{2} \zeta_{0}\left(\tau_{n} \xi\right)^{2}\left|\widehat{u_{n}}\left(\tau_{n} \xi\right)\right|^{2} \mathrm{~d} \xi=\int_{\mathbf{R}^{2}}|\xi|^{2} \zeta_{0}(\xi)^{2}\left|\widehat{u_{n}}\right|^{2} \mathrm{~d} \xi \leq \int_{|\xi| \leq 2}|\xi|^{2}\left|\widehat{u_{n}}\right|^{2} \mathrm{~d} \xi .
$$

Thus, by (23), $\left(w_{n, 1}\right)$ is bounded in $H_{\mathrm{r}}^{1}\left(\mathbf{R}^{2}\right)$ and suppose that $w_{n, 1} \rightarrow w_{0}$ weakly in $H^{1}\left(\mathbf{R}^{N}\right)$.

On the other hand, by

$$
\int_{\mathbf{R}^{2}}|\xi|^{2 \alpha}\left|\widehat{w_{n, 2}}\right|^{2} \mathrm{~d} \xi=\tau_{n}^{2-2 \alpha} \int_{\mathbf{R}^{2}}|\xi|^{2 \alpha}\left(1-\zeta_{0}(\xi)\right)^{2}\left|\widehat{u_{n}}(\xi)\right|^{2} \mathrm{~d} \xi \leq \tau_{n}^{2-2 \alpha} \int_{|\xi| \geq 1}|\xi|^{2 \alpha}\left|\widehat{u_{n}}\right|^{2} \mathrm{~d} \xi \rightarrow 0,
$$

it follows from (24) that $w_{n, 2} \rightarrow 0$ strongly in $H^{\alpha}\left(\mathbf{R}^{N}\right)$. Recalling $v_{n}=w_{n, 1}+w_{n, 2}$, we get

$$
v_{n} \rightarrow w_{0}\left(\in H^{1}\left(\mathbf{R}^{N}\right)\right) \quad \text { weakly in } H^{\alpha}\left(\mathbf{R}^{N}\right) .
$$

Now let $\varphi \in C_{0}^{\infty}\left(\mathbf{R}^{N}\right)$ be radial and set $\varphi_{n}(x):=\varphi\left(\tau_{n} x\right)$. Noting

$$
\left\|\tau_{n}^{2} \varphi_{n}\right\|_{\alpha}^{2}=\tau_{n}^{2} \int_{\mathbf{R}^{2}}\left(1+4 \pi^{2}|\xi|^{2} \tau_{n}^{2}\right)^{\alpha}|\widehat{\varphi}|^{2} \mathrm{~d} \xi \rightarrow 0
$$

and using $D_{u} \tilde{I}\left(u_{n}, 0\right) \rightarrow 0$, we infer that

$$
\begin{aligned}
\int_{\mathbf{R}^{2}} f\left(v_{n}\right) \varphi \mathrm{d} x & =\tau_{n}^{2} \int_{\mathbf{R}^{2}} f\left(u_{n}\right) \varphi_{n} \mathrm{~d} x=\tau_{n}^{2}\left\langle u_{n}, \varphi_{n}\right\rangle_{\alpha}+o\left(\left\|\tau_{n}^{2} \varphi_{n}\right\|_{\alpha}\right) \\
& =\int_{\mathbf{R}^{2}}\left(1+4 \pi^{2}|\xi|^{2} \tau_{n}^{2}\right)^{\alpha} \widehat{v_{n}} \bar{\varphi} \mathrm{d} \xi+o\left(\left\|\tau_{n}^{2} \varphi_{n}\right\|_{\alpha}\right) .
\end{aligned}
$$


Since $v_{n} \rightarrow w_{0}$ strongly in $L^{p}\left(\mathbf{R}^{N}\right)$ for $2<p<2_{\alpha}^{*}$ due to (25) and Lemma 2.1 (ii), by Strauss' lemma( [37, Lemma 2] or [7, Theorem A.I]), (f3) and $\varphi \in C_{0}^{\infty}\left(\mathbf{R}^{N}\right)$, one has

$$
\int_{\mathbf{R}^{2}} f\left(v_{n}\right) \varphi \mathrm{d} x \rightarrow \int_{\mathbf{R}^{2}} f\left(w_{0}\right) \varphi \mathrm{d} x .
$$

On the other hand, since $\widehat{\varphi}$ is rapidly decreasing, it follows that

$$
\lim _{n \rightarrow \infty} \int_{\mathbf{R}^{2}}\left(1+4 \pi^{2}|\xi|^{2} \tau_{n}^{2}\right)^{\alpha} \widehat{v_{n}} \bar{\varphi} \mathrm{d} \xi=\int_{\mathbf{R}^{2}} \widehat{w_{0}} \overline{\hat{\varphi}} \mathrm{d} \xi=\int_{\mathbf{R}^{2}} w_{0} \varphi \mathrm{d} x .
$$

Now by (26)-(28), we finally obtain

$$
\int_{\mathbf{R}^{2}} f\left(w_{0}\right) \varphi \mathrm{d} x=\int_{\mathbf{R}^{2}} w_{0} \varphi \mathrm{d} x
$$

for all radial $\varphi \in C_{0}^{\infty}\left(\mathbf{R}^{2}\right)$, which yields

$$
f\left(w_{0}\right)-w_{0} \equiv 0 \quad \text { in } \mathbf{R}^{2} .
$$

On the other hand, from (f2), one may find some $s_{1}>0$ such that $s(f(s)-s)<0$ for all $|s| \leq s_{1}$ with $s \neq 0$. Thus by (29), $w_{0} \in H_{\mathrm{r}}^{1}\left(\mathbf{R}^{N}\right) \subset C\left(\mathbf{R}^{N} \backslash\{0\}\right)$ and $w_{0}(x) \rightarrow 0$ as $|x| \rightarrow \infty$, we conclude that $w_{0} \equiv 0$ and Step 2 holds due to $(25)$.

\section{Step 3: Conclusion}

Now we derive a contradiction and conclude that $(21)$ holds. Since $\left(v_{n}\right)$ is bounded in $H^{\alpha}\left(\mathbf{R}^{N}\right)$ from Steps 1 and 2, we first remark that

$$
\left\|\tau_{n}^{N} u_{n}\right\|_{\alpha}^{2}=\int_{\mathbf{R}^{N}}\left(1+4 \pi^{2}|\xi|^{2}\right)^{\alpha}\left|\widehat{v_{n}}\left(\tau_{n}^{-1} \xi\right)\right|^{2} \mathrm{~d} \xi=\tau_{n}^{N} \int_{\mathbf{R}^{N}}\left(1+4 \pi^{2}|\xi|^{2} \tau_{n}^{2}\right)^{\alpha}\left|\widehat{v_{n}}\right|^{2} \mathrm{~d} \xi \rightarrow 0 .
$$

Let $\delta_{0}>0$ and $s_{1}>0$ be constants appearing in (10). It follows from $1=\left\|v_{n}\right\|_{L^{2}}^{2} \leq \tau_{n}^{N}\left\|u_{n}\right\|_{\alpha}^{2}$ and $D_{u} \tilde{I}\left(0, u_{n}\right) \rightarrow 0$ that

$$
\begin{aligned}
\delta_{0} & =\delta_{0}\left\|v_{n}\right\|_{L^{2}}^{2} \leq \tau_{n}^{N}\left\|u_{n}\right\|_{\alpha}^{2}-\left(1-\delta_{0}\right)\left\|v_{n}\right\|_{L^{2}}^{2} \\
& =\int_{\mathbf{R}^{N}} f\left(u_{n}\right) \tau_{n}^{N} u_{n} \mathrm{~d} x+o(1)-\left(1-\delta_{0}\right)\left\|v_{n}\right\|_{L^{2}}^{2} \\
& =\int_{\mathbf{R}^{N}} f\left(v_{n}\right) v_{n}-\left(1-\delta_{0}\right) v_{n}^{2} \mathrm{~d} x+o(1) \\
& \leq \int_{\mathbf{R}^{N}}\left(f\left(v_{n}\right) v_{n}-\left(1-\delta_{0}\right) v_{n}^{2}\right)_{+} \mathrm{d} x+o(1) .
\end{aligned}
$$

By (10), we observe $\left(f(s) s-\left(1-\delta_{0}\right) s^{2}\right)_{+}=0$ for $|s| \leq s_{1}$. Hence, arguing as in the proof of Lemma 2.2 (v), it follows from (30) and $v_{n} \rightarrow 0$ weakly in $H_{\mathrm{r}}^{\alpha}\left(\mathbf{R}^{N}\right)$ due to Steps 1 and 2 that

$$
\delta_{0} \leq \int_{\mathbf{R}^{N}}\left(f\left(v_{n}\right) v_{n}-\left(1-\delta_{0}\right) v_{n}^{2}\right)_{+} \mathrm{d} x+o(1) \rightarrow 0
$$

which is a contradiction. Thus (21) holds and we complete the proof.

Now we prove the existence of critical points of $I$ which satisfy the Pohozaev identity $P(u)=0$ and correspond to $c_{n}$ in $(18)$.

Proposition 3.2. There exist a sequence $\left(u_{n}\right) \subset H_{\mathrm{r}}^{\alpha}\left(\mathbf{R}^{N}\right)$ such that $I^{\prime}\left(u_{n}\right)=0, I\left(u_{n}\right)=c_{n}$ and $P\left(u_{n}\right)=0$. Especially, (4) has infinitely many solutions satisfying the Pohozaev identity.

Proof. By Lemma 2.5, we have $c_{n}=\tilde{c}_{n}$. Hence, there exists a sequence $\left(\gamma_{n, k}\right) \subset \Gamma_{n}$ such that

$$
\max _{\sigma \in D_{n}} \tilde{I}\left(0, \gamma_{n, k}(\sigma)\right)=\max _{\sigma \in D_{n}} I\left(\gamma_{n, k}(\sigma)\right) \rightarrow \tilde{c}_{n} .
$$

Applying Ekeland's variational principle to $\left(\gamma_{n, k}\right)$ and $\tilde{I}$, there exist $\left(\theta_{n, k}, u_{n, k}\right) \in \mathbf{R} \times H_{\mathrm{r}}^{\alpha}\left(\mathbf{R}^{N}\right)$ such that

$$
\operatorname{dist}\left(\left(\theta_{n, k}, u_{n, k}\right),\{0\} \times \gamma_{n, k}\left(D_{n}\right)\right) \rightarrow 0, \quad \tilde{I}\left(\theta_{n, k}, u_{n, k}\right) \rightarrow \tilde{c}_{n}, \quad D_{(\theta, u)} \tilde{I}\left(\theta_{n, k}, u_{n, k}\right) \rightarrow 0 .
$$

In particular, $\theta_{n, k} \rightarrow 0$. Thus by Proposition $3.1,\left(u_{n, k}\right)_{k}$ is bounded in $H_{\mathrm{r}}^{\alpha}\left(\mathbf{R}^{N}\right)$. 
Now assume $u_{n, k} \rightarrow u_{n, 0}$ weakly in $H_{\mathrm{r}}^{\alpha}\left(\mathbf{R}^{N}\right)$ and $u_{n, k} \rightarrow u_{n, 0}$ strongly in $L^{p}\left(\mathbf{R}^{N}\right)$ for $2<p<2_{\alpha}^{*}$. Let $\delta_{0}, s_{1}>0$ be constants in (10). By (f4), $\sup _{s \in[0, \infty)}\left(f(s)-\left(1-\delta_{0}\right) s\right)>0$. Since $f(s)$ is odd, we may find an $s_{+}>0$ satisfying

$$
f\left( \pm s_{+}\right)-\left(1-\delta_{0}\right)\left( \pm s_{+}\right)=0, \quad f(s)-\left(1-\delta_{0}\right) s \neq 0 \quad \text { for } s \in\left(-s_{+}, s_{+}\right) \backslash\{0\} .
$$

Set $f_{1}(s):=f(s)-\left(1-\delta_{0}\right) s$ if $s \in\left[-s_{+}, s_{+}\right]$and $f_{1}(s):=0$ otherwise, and $f_{2}(s):=f(s)-\left(1-\delta_{0}\right) s-f_{1}(s)$. Remark that $s f_{1}(s) \leq 0$ for all $s \in \mathbf{R}$ and $f_{2}(s) s=0$ for all $s \in\left[-s_{+}, s_{+}\right]$.

By $D_{u} \tilde{I}\left(u_{n, k}\right) \rightarrow 0$, one can check $I^{\prime}\left(u_{n, 0}\right)=0$. Moreover, note that a norm defined by

$$
\|u\|^{2}:=\|u\|_{\alpha}^{2}-\left(1-\delta_{0}\right)\|u\|_{L^{2}}^{2}
$$

is equivalent to $\|\cdot\|_{\alpha}$. Thus, arguing as in Step 3 of Proposition 3.1 (see also the proof of Lemma $2.2(\mathrm{v})$ ), from the boundedness of $\left(u_{n, k}\right), D_{u} \tilde{I}\left(\theta_{n_{k}}, u_{n_{k}}\right) \rightarrow 0, \theta_{n_{k}} \rightarrow 0$, Fatou's lemma to $f_{1}\left(u_{n, k}\right) u_{n, k}$, properties of $f_{i}(s)$ $(i=1,2), I^{\prime}\left(u_{n, 0}\right)=0$ and the weak convergence of $\left(u_{n, k}\right)$, we observe that

$$
\begin{aligned}
\limsup _{k \rightarrow \infty}\left\|u_{n, k}\right\|^{2} & =\limsup _{k \rightarrow \infty} \int_{\mathbf{R}^{N}} f\left(u_{n, k}\right) u_{n, k}-\left(1-\delta_{0}\right) u_{n, k}^{2} \mathrm{~d} x \\
& \leq \limsup _{k \rightarrow \infty} \int_{\mathbf{R}^{N}} f_{1}\left(u_{n, k}\right) u_{n, k} \mathrm{~d} x+\limsup _{k \rightarrow \infty} \int_{\mathbf{R}^{N}} f_{2}\left(u_{n, k}\right) u_{n, k} \mathrm{~d} x \\
& \leq \int_{\mathbf{R}^{N}} f_{1}\left(u_{n, 0}\right) u_{n, 0} \mathrm{~d} x+\int_{\mathbf{R}^{N}} f_{2}\left(u_{n, 0}\right) u_{n, 0} \mathrm{~d} x \\
& =\int_{\mathbf{R}^{N}} f\left(u_{n, 0}\right) u_{n, 0}-\left(1-\delta_{0}\right) u_{n, 0}^{2} \mathrm{~d} x=\left\|u_{n, 0}\right\|^{2} \leq \liminf _{k \rightarrow \infty}\left\|u_{n, k}\right\|^{2} .
\end{aligned}
$$

This implies that $u_{n, k} \rightarrow u_{n, 0}$ strongly in $H_{\mathrm{r}}^{\alpha}\left(\mathbf{R}^{N}\right)$. Therefore, $I\left(u_{n, k}\right) \rightarrow \tilde{c}_{n}=c_{n}=I\left(u_{n, 0}\right)$ and $I^{\prime}\left(u_{n, 0}\right)=0$. Moreover, recalling (17), we have

$$
\lim _{k \rightarrow \infty} D_{\theta} \tilde{I}\left(\theta_{n, k}, u_{n, k}\right) \rightarrow 0=D_{\theta} \tilde{I}\left(0, u_{n, 0}\right)=P\left(u_{n, 0}\right) .
$$

This completes the proof.

By Proposition 3.2, a set

$$
S:=\left\{u \in H^{\alpha}\left(\mathbf{R}^{N}\right) \mid u \not \equiv 0, I^{\prime}(u)=0, P(u)=0\right\}
$$

is not empty. Moreover, we have $c_{\mathrm{LES}} \leq c_{1}$. Next we show

Proposition 3.3. For every $u \in H^{\alpha}\left(\mathbf{R}^{N}\right)$ with $P(u)=0$ and $u \not \equiv 0$, a path $\gamma_{u}(t):=u(x / t)$ for $t>0$ and $\gamma_{u}(0):=0$ satisfies

$$
\gamma_{u} \in C\left([0, \infty), H^{\alpha}\left(\mathbf{R}^{N}\right)\right), \quad I(u)>I\left(\gamma_{u}(t)\right) \quad \text { for any } t \neq 1, \quad I\left(\gamma_{u}(t)\right) \rightarrow-\infty \quad \text { as } t \rightarrow \infty .
$$

Proof. For $t>0$, one sees

$$
\left\|\gamma_{u}(t)\right\|_{L^{2}}^{2}=t^{N}\|u\|_{L^{2}}^{2}, \quad\left\||\xi|^{\alpha} \widehat{\gamma_{u}(t)}\right\|_{L^{2}}^{2}=t^{N-2 \alpha} \int_{\mathbf{R}^{N}}|\xi|^{2 \alpha}|\widehat{u}|^{2} \mathrm{~d} \xi .
$$

Thus $\gamma_{u} \in C\left([0, \infty), H^{\alpha}\left(\mathbf{R}^{N}\right)\right)$. Furthermore, it follows from $I\left(\gamma_{u}(t)\right)=\tilde{I}(\log t, u)$ and $(17)$ that

$$
\begin{aligned}
& \frac{\mathrm{d}}{\mathrm{d} t} I\left(\gamma_{u}(t)\right)=D_{\theta} \tilde{I}(\log t, u) \frac{1}{t} \\
&=t^{N-1}\left\{\frac{N}{2} \int_{\mathbf{R}^{N}}\left(1+4 \pi^{2} \frac{|\xi|^{2}}{t^{2}}\right)^{\alpha}|\widehat{u}|^{2} \mathrm{~d} \xi-N \int_{\mathbf{R}^{N}} F(u) \mathrm{d} x\right. \\
&\left.-\alpha \int_{\mathbf{R}^{N}}\left(1+4 \pi^{2} \frac{|\xi|^{2}}{t^{2}}\right)^{\alpha-1} 4 \pi^{2} \frac{|\xi|^{2}}{t^{2}}|\widehat{u}|^{2} \mathrm{~d} \xi\right\} \\
&=t^{N-1}\left\{\frac{N-2 \alpha}{2} \int_{\mathbf{R}^{N}}\left(1+4 \pi^{2} \frac{|\xi|^{2}}{t^{2}}\right)^{\alpha}|\widehat{u}|^{2} \mathrm{~d} \xi-N \int_{\mathbf{R}^{N}} F(u) \mathrm{d} x\right. \\
&\left.+\alpha \int_{\mathbf{R}^{N}}\left(1+4 \pi^{2} \frac{|\xi|^{2}}{t^{2}}\right)^{\alpha-1}|\widehat{u}|^{2} \mathrm{~d} \xi\right\} .
\end{aligned}
$$

Now set

$$
g(t):=\frac{N-2 \alpha}{2} \int_{\mathbf{R}^{N}}\left(1+4 \pi^{2} \frac{|\xi|^{2}}{t^{2}}\right)^{\alpha}|\widehat{u}|^{2} \mathrm{~d} \xi-N \int_{\mathbf{R}^{N}} F(u) \mathrm{d} x+\alpha \int_{\mathbf{R}^{N}}\left(1+4 \pi^{2} \frac{|\xi|^{2}}{t^{2}}\right)^{\alpha-1}|\widehat{u}|^{2} \mathrm{~d} \xi .
$$


Then we get

$$
\begin{aligned}
g^{\prime}(t)= & \frac{N-2 \alpha}{2} \alpha \int_{\mathbf{R}^{N}}\left(1+4 \pi^{2} \frac{|\xi|^{2}}{t^{2}}\right)^{\alpha-1}(-2) \frac{4 \pi^{2}|\xi|^{2}}{t^{3}}|\widehat{u}|^{2} \mathrm{~d} \xi \\
& +\alpha(\alpha-1) \int_{\mathbf{R}^{N}}\left(1+4 \pi^{2} \frac{|\xi|^{2}}{t^{2}}\right)^{\alpha-2}(-2) \frac{4 \pi^{2}|\xi|^{2}}{t^{3}}|\widehat{u}|^{2} \mathrm{~d} \xi \\
= & -2 \alpha \int_{\mathbf{R}^{N}}\left(1+4 \pi^{2} \frac{|\xi|^{2}}{t^{2}}\right)^{\alpha-2} \frac{4 \pi^{2}|\xi|^{2}}{t^{3}}|\widehat{u}|^{2}\left\{\frac{N-2 \alpha}{2}\left(1+4 \pi^{2} \frac{|\xi|^{2}}{t^{2}}\right)+(\alpha-1)\right\} \mathrm{d} \xi \\
= & -2 \alpha \int_{\mathbf{R}^{N}}\left(1+4 \pi^{2} \frac{|\xi|^{2}}{t^{2}}\right)^{\alpha-2} \frac{4 \pi^{2}|\xi|^{2}}{t^{3}}|\widehat{u}|^{2}\left\{\left(\frac{N}{2}-1\right)+\frac{2 \pi^{2}(N-2 \alpha)|\xi|^{2}}{t^{2}}\right\} \mathrm{d} \xi .
\end{aligned}
$$

Since $N \geq 2$ and $N>2 \alpha$, one has $g^{\prime}(t)<0$ for all $t>0$. Noting that $\mathrm{d} I\left(\gamma_{u}(t)\right) / \mathrm{d} t=t^{N-1} g(t)$ and that $P(u)=0$ is equivalent to $g(1)=0$, we see that

$$
\frac{\mathrm{d}}{\mathrm{d} t} I\left(\gamma_{u}(t)\right)>0 \quad \text { if } 0<t<1, \quad \frac{\mathrm{d}}{\mathrm{d} t} I\left(\gamma_{u}(t)\right)<0 \quad \text { if } 1<t,
$$

which implies that $I\left(\gamma_{u}(t)\right)$ has a unique maximum at $t=1$. From the monotonicity of $g(t)$ and $g(1)=0$, it is clear that $I\left(\gamma_{u}(t)\right) \rightarrow-\infty$ as $t \rightarrow \infty$ and Proposition 3.3 holds.

Before proceeding to a proof of $c_{1}=c_{\mathrm{LES}}$, we define the following quantities:

$$
\begin{aligned}
c_{\mathrm{MP}, \mathrm{r}} & :=\inf _{\gamma \in \Gamma_{\mathrm{r}}} \max _{0 \leq t \leq 1} I(\gamma(t)), \quad \Gamma_{\mathrm{r}}:=\left\{\gamma \in C\left([0,1], H_{\mathrm{r}}^{\alpha}\left(\mathbf{R}^{N}\right) \mid \gamma(0)=0, I(\gamma(1))<0\right\},\right. \\
c_{\mathrm{MP}} & :=\inf _{\gamma \in \Gamma} \max _{0 \leq t \leq 1} I(\gamma(t)), \quad \Gamma:=\left\{\gamma \in C\left([0,1], H^{\alpha}\left(\mathbf{R}^{N}\right) \mid \gamma(0)=0, I(\gamma(1))<0\right\} .\right. \\
\tilde{c}_{\mathrm{MP}, \mathrm{r}} & :=\inf _{\tilde{\gamma} \in \tilde{\Gamma}_{\mathrm{r}} \max _{0 \leq t \leq 1} \tilde{I}(\tilde{\gamma}(t)),} \\
\tilde{\Gamma}_{\mathrm{r}} & :=\left\{\tilde{\gamma}=(\theta, \gamma) \in C\left([0,1], \mathbf{R} \times H_{\mathrm{r}}^{\alpha}\left(\mathbf{R}^{N}\right)\right) \mid \gamma \in \Gamma_{\mathrm{r}}, \theta(0)=0=\theta(1)\right\} .
\end{aligned}
$$

Then we show

Lemma 3.4. $0<c_{\mathrm{MP}}=c_{\mathrm{MP}, \mathrm{r}}=c_{1}=c_{\mathrm{LES}}=\tilde{c}_{\mathrm{MP}, \mathrm{r}}$.

Proof. As in the proof of Lemma 2.5, one sees $\tilde{c}_{\mathrm{MP}, \mathrm{r}}=c_{\mathrm{MP}, \mathrm{r}}$. Moreover, from the definition, $c_{\mathrm{MP}} \leq c_{\mathrm{MP}, \mathrm{r}} \leq c_{1}$. In addition, by Propositions 3.2 and 3.3, we know $c_{\mathrm{MP}} \leq c_{\mathrm{LES}} \leq c_{1}$. Thus it is sufficient to prove $c_{1} \leq c_{\mathrm{MP}}$.

We first claim that

$$
c_{\mathrm{MP}}=d:=\inf _{\eta \in \bar{\Gamma}} \max _{0 \leq t \leq 1} I(\eta(t)), \quad \bar{\Gamma}:=\left\{\eta \in C\left([0,1], H^{\alpha}\left(\mathbf{R}^{N}\right)\right) \mid \eta(0)=0, \eta(1)=\gamma_{1}(1)\right\}
$$

where $\gamma_{1}$ appears in Lemma 2.3. From the definition of $\Gamma$ and $I\left(\gamma_{1}(1)\right)<0$, we have $c_{\mathrm{MP}} \leq d$. For the opposite inequality $d \leq c_{\mathrm{MP}}$, it is enough to show that $[I<0]:=\left\{u \in H^{\alpha}\left(\mathbf{R}^{N}\right) \mid I(u)<0\right\}$ is path-connected in $H^{\alpha}\left(\mathbf{R}^{N}\right)$. A similar claim is proved in [24] for the case $\alpha=1$ and we use the same argument.

Let $u_{1}, u_{2} \in[I<0]$. Since $C_{0}^{\infty}\left(\mathbf{R}^{N}\right)$ is dense in $H^{\alpha}\left(\mathbf{R}^{N}\right)$, we may assume $u_{1}, u_{2} \in C_{0}^{\infty}\left(\mathbf{R}^{N}\right)$. For $u_{i}$, we consider the path $\gamma_{i}(t)=\gamma_{u_{i}}(t)$ appearing in Proposition 3.3. From the computations in the proof of Proposition 3.3 , we observe that $\mathrm{d} I\left(\gamma_{i}(t)\right) / \mathrm{d} t>0$ if $0<t \ll 1$. Since $I\left(\gamma_{i}(0)\right)=0>I\left(\gamma_{i}(1)\right)=I\left(u_{i}\right)$, there are maximum points $t_{i} \in(0,1)$ of $I\left(\gamma_{i}(t)\right)$ with $I\left(\gamma_{i}\left(t_{i}\right)\right)>0$. At those points, we have $\mathrm{d} I\left(\gamma_{i}(t)\right) /\left.\mathrm{d} t\right|_{t=t_{i}}=0$, which yields $P\left(\gamma_{i}\left(t_{i}\right)\right)=0$. Hence, by Proposition 3.3, we observe that $t \mapsto I\left(\gamma_{i}(t)\right):\left(t_{i}, \infty\right) \rightarrow \mathbf{R}$ is strictly decreasing and $I\left(\gamma_{i}(t)\right) \rightarrow-\infty$ as $t \rightarrow \infty$. Thus choose $t_{0}>1$ so large that

$$
I\left(\gamma_{i}\left(t_{0}\right)\right)<-2 \max \left\{\max _{0 \leq s \leq 1} I\left(s u_{1}\right), \max _{0 \leq s \leq 1} I\left(s u_{2}\right)\right\}<0 .
$$

Noting that $\gamma_{i}\left(t_{0}\right)(x)=u_{i}\left(x / t_{0}\right), u_{i} \in C_{0}^{\infty}\left(\mathbf{R}^{N}\right)$ and

$$
\begin{aligned}
\left\langle u_{1}\left(x / t_{0}\right), s u_{2}\left(x-R \mathbf{e}_{1}\right)\right\rangle_{\alpha} & \rightarrow 0 \\
\int_{\mathbf{R}^{N}} F\left(u_{1}\left(x / t_{0}\right)+s u_{2}\left(x-R \mathbf{e}_{1}\right)\right) \mathrm{d} x & \rightarrow \int_{\mathbf{R}^{N}} F\left(u_{1} / t_{0}\right) \mathrm{d} x+\int_{\mathbf{R}^{N}} F\left(s u_{2}(x)\right) \mathrm{d} x
\end{aligned}
$$

uniformly with respect to $s \in[0,1]$ as $R \rightarrow \infty$ where $\mathbf{e}_{1}=(1,0, \ldots, 0)$, it follows from the choice of $t_{0}$ that as $R \rightarrow \infty$

$$
\max _{0 \leq s \leq 1} I\left(\gamma_{1}\left(t_{0}\right)+s u_{2}\left(x-R \mathbf{e}_{1}\right)\right) \rightarrow I\left(\gamma_{1}\left(t_{0}\right)\right)+\max _{0 \leq s \leq 1} I\left(s u_{2}\right)<-\max _{0 \leq s \leq 1} I\left(s \gamma_{2}\left(t_{0}\right)\right)<0
$$


Hence, choosing $R_{0}>0$ so large, we have

$$
\begin{aligned}
& \operatorname{supp} \gamma_{1}(t) \cap \operatorname{supp} u_{2}\left(\cdot-R_{0} \mathbf{e}_{1}\right)=\emptyset \quad \text { for } 1 \leq t \leq t_{0}, \quad \max _{0 \leq s \leq 1} I\left(\gamma_{1}\left(t_{0}\right)+s u_{2}\left(\cdot-R_{0} \mathbf{e}_{1}\right)\right)<0, \\
& I\left(\gamma_{1}(t)+u_{2}\left(\cdot-R_{0} \mathbf{e}_{1}\right)\right)=I\left(\gamma_{1}(t)\right)+I\left(u_{2}\right)<0 \quad \text { for } 1 \leq t \leq t_{0} .
\end{aligned}
$$

Through the paths $t \mapsto \gamma_{1}(t)\left(t \in\left[1, t_{0}\right]\right), s \mapsto \gamma_{1}\left(t_{0}\right)(x)+s u_{2}\left(x-R_{0} \mathbf{e}_{1}\right)(s \in[0,1])$ and $\theta \mapsto \gamma_{1}\left(t_{0}-\theta\right)(x)+$ $u_{2}\left(x-R_{0} \mathbf{e}_{1}\right)\left(\theta \in\left[0, t_{0}-1\right]\right)$, we can connect $u_{1}$ and $u_{1}(x)+u_{2}\left(x-R_{0} \mathbf{e}_{1}\right)$ in $[I<0]$. In a similar fashion, we see that there is a path between $u_{2}$ and $u_{1}(x)+u_{2}\left(x-R_{0} \mathbf{e}_{1}\right)$ in $[I<0]$. Therefore, $[I<0]$ is path-connected in $H^{\alpha}\left(\mathbf{R}^{N}\right)$ and $c_{\mathrm{MP}}=d$ follows.

Next, since $I(u)=I(-u), \gamma_{1}(1) \in H_{\mathrm{r}}^{\alpha}\left(\mathbf{R}^{N}\right)$ and $\gamma(-t)=-\gamma(t)$ holds for any $\gamma \in \Gamma_{1}$, it suffices to prove

$$
d=\inf _{\eta \in \bar{\Gamma}_{\mathrm{r}}} \max _{0 \leq t \leq 1} I(\eta(t))\left(=c_{1}\right), \quad \bar{\Gamma}_{\mathrm{r}}:=\left\{\eta \in C\left([0,1], H_{\mathrm{r}}^{\alpha}\left(\mathbf{R}^{N}\right)\right) \mid \eta(0)=0, \eta(1)=\gamma_{1}(1)\right\} .
$$

By definition, one has $d \leq c_{1}$.

On the other hand, let $\eta \in \bar{\Gamma}$. Since $F(s)$ is even, we have $\int_{\mathbf{R}^{N}} F(u) \mathrm{d} x=\int_{\mathbf{R}^{N}} F(|u|) \mathrm{d} x$ for each $u \in H^{\alpha}\left(\mathbf{R}^{N}\right)$. Moreover, noting the following inequality (see Lemma A.3)

$$
\||u|\|_{\alpha} \leq\|u\|_{\alpha} \text { for all } u \in H^{\alpha}\left(\mathbf{R}^{N}\right),
$$

we observe that $\zeta(t)(x):=|\eta(t)(x)|$ satisfies

$$
I(\zeta(t)) \leq I(\eta(t)) \quad \text { for all } t \in[0,1], \quad \zeta \in C\left([0,1], H^{\alpha}\left(\mathbf{R}^{N}\right)\right) .
$$

Recalling Remark 2.4, we have $\zeta \in \bar{\Gamma}$.

Now set $\tilde{\zeta}(t)(x):=(\zeta(t))^{*}(x)$ where $u^{*}$ denotes the Schwarz symmetrization of $u$. By [1, Theorem 9.2], we have $\tilde{\zeta} \in C\left([0,1], H_{\mathrm{r}}^{\alpha}\left(\mathbf{R}^{N}\right)\right)$ and $\int_{\mathbf{R}^{N}} F(\tilde{\zeta}(t)) \mathrm{d} x=\int_{\mathbf{R}^{N}} F(\zeta(t)) \mathrm{d} x$. Moreover, from Remark 2.4, it follows that $\left(\gamma_{1}(1)\right)^{*}=\gamma_{1}(1)$, which yields $\tilde{\zeta} \in \bar{\Gamma}_{\mathrm{r}}$. Since $\|\tilde{\zeta}(t)\|_{\alpha} \leq\|\zeta(t)\|_{\alpha}$ holds thanks to [34, Proposition 4], we see that

$$
c_{1} \leq \max _{0 \leq t \leq 1} I(\tilde{\zeta}(t)) \leq \max _{0 \leq t \leq 1} I(\zeta(t)) \leq \max _{0 \leq t \leq 1} I(\eta(t))
$$

Since $\eta$ is any element of $\bar{\Gamma}$, one sees $c_{1} \leq d$.

Therefore, from (32) and (33), we get $c_{\mathrm{MP}}=d=c_{1}$ and this completes the proof.

Finally we shall show that if either $\alpha>1 / 2$ or $f(s)$ is locally Lipschitz, then every weak solution of (4) satisfies $P(u)=0$. To this end, we use the following Brézis-Kato type result [9]:

Proposition 3.5. Assume that $u \in H^{\alpha}\left(\mathbf{R}^{N}\right)$ is a weak solution of

$$
(1-\Delta)^{\alpha} u-a(x) u=0 \quad \text { in } \mathbf{R}^{N}
$$

where $a(x)$ satisfies

$$
|a(x)| \leq C_{0}(1+A(x)) \quad \text { for a.e. } x \in \mathbf{R}^{N}, \quad A \in L^{N /(2 \alpha)}\left(\mathbf{R}^{N}\right) .
$$

Then $u \in L^{p}\left(\mathbf{R}^{N}\right)$ for all $p \in[2, \infty)$.

We give a sketch of proof for Proposition 3.5 in Appendix. For related results, see [21]. Using Proposition 3.5, we shall prove

Proposition 3.6. (i) Let $u \in H^{\alpha}\left(\mathbf{R}^{N}\right)$ be a weak solution of (4). Then $u \in C_{\mathrm{b}}^{\beta}\left(\mathbf{R}^{N}\right)$ for every $\beta \in(0,2 \alpha)$ and $u$ decays faster than any polynomial, i.e., for any $k \in \mathbf{N}$ there exists a $c_{k}>0$ such that $|u(x)| \leq c_{k}(1+|x|)^{-k}$ for all $x \in \mathbf{R}^{N}$. Here

$$
\begin{aligned}
& C_{\mathrm{b}}^{\beta}\left(\mathbf{R}^{N}\right):=\left\{u \in C\left(\mathbf{R}^{N}\right) \cap L^{\infty}\left(\mathbf{R}^{N}\right) \mid[u]_{C^{\beta}}:=\sup _{x, y \in \mathbf{R}^{N}, x \neq y} \frac{|u(x)-u(y)|}{|x-y|^{\beta}}<\infty\right\} \quad \text { if } \beta<1, \\
& C_{\mathrm{b}}^{1}\left(\mathbf{R}^{N}\right):=\left\{u \in C^{1}\left(\mathbf{R}^{N}\right) \mid u, \nabla u \in L^{\infty}\left(\mathbf{R}^{N}\right)\right\}, \\
& C_{\mathrm{b}}^{\beta}\left(\mathbf{R}^{N}\right):=\left\{u \in C_{\mathrm{b}}^{1}\left(\mathbf{R}^{N}\right) \mid \nabla u \in C_{\mathrm{b}}^{\beta-1}\left(\mathbf{R}^{N}\right)\right\} \quad \text { if } 1<\beta<2 .
\end{aligned}
$$

(ii) In addition to (f1)-(f4), assume that $f(s)$ is locally Lipschitz continuous and $0<\alpha \leq 1 / 2$. Then $u \in$ $C_{\mathrm{b}}^{1+\beta}\left(\mathbf{R}^{N}\right)$ for all $\beta \in(0,2 \alpha)$.

(iii) Assume that $u \in H_{\mathrm{r}}^{\alpha}\left(\mathbf{R}^{N}\right) \cap C_{\mathrm{b}}^{1}\left(\mathbf{R}^{N}\right)$ is a weak solution of (4). Then $P(u)=0$.

A proof of (iii) below is essentially due to [23]. See also [34]. 
Proof. (i) Let $u \in H^{\alpha}\left(\mathbf{R}^{N}\right)$ be a weak solution of (4) and set $a(x):=f(u(x)) / u(x)$. From (f2) and (f3), it follows that

$$
|a(x)| \leq C\left(1+|u(x)|^{2_{\alpha}^{*}-2}\right) \quad \text { for a.e. } x \in \mathbf{R}^{N} .
$$

Noting that $u$ is a weak solution of $(1-\Delta)^{\alpha} u-a(x) u=0$ in $\mathbf{R}^{N}$, Proposition 3.5 yields $u \in L^{p}\left(\mathbf{R}^{N}\right)$ for all $p \in[2, \infty)$. Using (f2) and (f3) again, we observe that $f(u(x)) \in L^{p}\left(\mathbf{R}^{N}\right)$ for any $p \in[2, \infty)$. Thus, recalling the argument in Lemma 2.1, we have

$$
u=G_{2 \alpha} * f(u), \quad u \in \mathscr{L}_{2 \alpha}^{p}:=\left\{G_{2 \alpha} * g \mid g \in L^{p}\left(\mathbf{R}^{N}\right)\right\} \quad \text { for all } p \in[2, \infty) .
$$

Since $\mathscr{L}_{2 \alpha}^{p} \subset W^{2 \alpha, p}\left(\mathbf{R}^{N}\right)$ (see [35]), Sobolev's inequality yields $u \in C_{\mathrm{b}}^{\beta}\left(\mathbf{R}^{N}\right)$ for each $\beta \in(0,2 \alpha)$. Hence, the first assertion in (i) holds.

For the decay estimate, let $\delta_{0}>0$ be a constant appearing in (10) and $v \in H^{\alpha}\left(\mathbf{R}^{N}\right)$ a unique weak solution of

$$
(1-\Delta)^{\alpha} v-\left(1-\delta_{0}\right) v=\left(f(u)-\left(1-\delta_{0}\right) u\right)_{+}=: g(x) \text { in } \mathbf{R}^{N} .
$$

Since $u \in C_{\mathrm{b}}^{\beta}\left(\mathbf{R}^{N}\right)$, we have $u(x) \rightarrow 0$ as $|x| \rightarrow \infty$ and $g(x) \in L^{\infty}\left(\mathbf{R}^{N}\right)$ has compact support. Hence, by Proposition A.1 (ii), for any $k \in \mathbf{N}$, $v$ satisfies $v(x) \leq c_{k}(1+|x|)^{-k}$ for $x \in \mathbf{R}^{N}$. Moreover, rewriting (4) as

$$
(1-\Delta)^{\alpha} u-\left(1-\delta_{0}\right) u=f(u)-\left(1-\delta_{0}\right) u \text { in } \mathbf{R}^{N},
$$

Proposition A.1 (iii) asserts $u(x) \leq v(x) \leq c_{k}(1+|x|)^{-k}$ for all $x \in \mathbf{R}^{N}$.

On the other hand, let $w$ be a solution of

$$
(1-\Delta)^{\alpha} w-\left(1-\delta_{0}\right) w=\left(f(u)-\left(1-\delta_{0}\right) u\right)_{-} \quad \text { in } \mathbf{R}^{N} .
$$

Since

$$
(1-\Delta)^{\alpha}(-u)-\left(1-\delta_{0}\right)(-u)=-\left(f(u)-\left(1-\delta_{0}\right) u\right) \leq(1-\Delta)^{\alpha} w-\left(1-\delta_{0}\right) w \quad \text { in } \mathbf{R}^{N},
$$

using Proposition A.1 again, we get $-u(x) \leq w(x) \leq c_{k}(1+|x|)^{-k}$ for $x \in \mathbf{R}^{N}$. Thus (i) holds.

(ii) Let $f(s)$ be locally Lipschitz and $0<\alpha \leq 1 / 2$. Since $u \in C_{\mathrm{b}}^{\beta}\left(\mathbf{R}^{N}\right)$ for $0<\beta<2 \alpha$ by (i), we have

$$
\left|f\left(u\left(x_{1}\right)\right)-f\left(u\left(x_{2}\right)\right)\right| \leq L\left|u\left(x_{1}\right)-u\left(x_{2}\right)\right| \leq L[u]_{C^{\beta}}\left|x_{1}-x_{2}\right|^{\beta} .
$$

Thus $f(u(x)) \in C_{\mathrm{b}}^{\beta}\left(\mathbf{R}^{N}\right)$. Since $u=G_{2 \alpha} * f(u)$ and $C_{\mathrm{b}}^{\beta+2 \alpha}\left(\mathbf{R}^{N}\right)=G_{2 \alpha} * C_{\mathrm{b}}^{\beta}\left(\mathbf{R}^{N}\right)$ holds by [35, Theorem 4 in $\S 5$ of Chapter V], applying the bootstrap argument, we can check that $u \in C_{\mathrm{b}}^{\beta}\left(\mathbf{R}^{N}\right)$ for all $\beta<1+2 \alpha$.

(iii) We follow the argument in $[23,34]$. Let $u \in H^{\alpha}\left(\mathbf{R}^{N}\right) \cap C_{\mathrm{b}}^{1}\left(\mathbf{R}^{N}\right)$ be a weak solution of (4). For a mollifier $\left(\rho_{\varepsilon}\right)$, set $u_{\varepsilon}(x):=u * \rho_{\varepsilon}$. Thanks to the decay estimate of $u$, we observe that $u_{\varepsilon} \in \mathscr{S}\left(\mathbf{R}^{N}, \mathbf{R}\right)$. Thus,

$$
\left\langle u, x \cdot \nabla u_{\varepsilon}\right\rangle_{\alpha}=\int_{\mathbf{R}^{N}} f(u) x \cdot \nabla u_{\varepsilon} \mathrm{d} x .
$$

Noting

$$
\begin{aligned}
& \left\langle u, x \cdot \nabla u_{\varepsilon}\right\rangle_{\alpha}=\int_{\mathbf{R}^{N}} \widehat{u}(\xi) \overline{\left(1+4 \pi^{2}|\xi|^{2}\right)^{\alpha} \widehat{x \cdot \nabla u_{\varepsilon}}} \mathrm{d} \xi=\int_{\mathbf{R}^{N}} u(x)(1-\Delta)^{\alpha}\left(x \cdot \nabla u_{\varepsilon}\right) \mathrm{d} x, \\
& (1-\Delta)^{\alpha} u_{\varepsilon}=(1-\Delta)^{\alpha}\left(\rho_{\varepsilon} * u\right)=\rho_{\varepsilon} *(1-\Delta)^{\alpha} u=\rho_{\varepsilon} * f(u),
\end{aligned}
$$

and using ( [23, Proposition 5.1])

$$
(1-\Delta)^{\alpha}\left(x \cdot \nabla u_{\varepsilon}\right)=x \cdot \nabla\left[(1-\Delta)^{\alpha} u_{\varepsilon}\right]+2 \alpha(1-\Delta)^{\alpha} u_{\varepsilon}-2 \alpha(1-\Delta)^{\alpha-1} u_{\varepsilon},
$$

we obtain

$$
\begin{aligned}
\left\langle u, x \cdot \nabla u_{\varepsilon}\right\rangle_{\alpha} & =\int_{\mathbf{R}^{N}} u(x)(1-\Delta)^{\alpha}\left(x \cdot \nabla u_{\varepsilon}\right) \\
& =\int_{\mathbf{R}^{N}} u(x)\left[x \cdot \nabla\left(\rho_{\varepsilon} * f(u)\right)+2 \alpha \rho_{\varepsilon} * f(u)-2 \alpha(1-\Delta)^{\alpha-1} u_{\varepsilon}\right] \mathrm{d} x .
\end{aligned}
$$

By $u_{\varepsilon} \rightarrow u$ strongly in $L^{2}\left(\mathbf{R}^{N}\right)$ and $0<\alpha<1$, it is easily seen that

$$
\begin{aligned}
& \lim _{\varepsilon \rightarrow 0} \int_{\mathbf{R}^{N}} u \rho_{\varepsilon} * f(u) \mathrm{d} x=\lim _{\varepsilon \rightarrow 0} \int_{\mathbf{R}^{N}}\left(u * \rho_{\varepsilon}\right) f(u) \mathrm{d} x=\int_{\mathbf{R}^{N}} u f(u) \mathrm{d} x=\|u\|_{\alpha}^{2}, \\
& \lim _{\varepsilon \rightarrow 0} \int_{\mathbf{R}^{N}}(1-\Delta)^{\alpha-1} u_{\varepsilon} u \mathrm{~d} x=\lim _{\varepsilon \rightarrow 0} \int_{\mathbf{R}^{N}}\left(1+4 \pi^{2}|\xi|^{2}\right)^{\alpha-1} \widehat{u_{\varepsilon}} \overline{\widehat{u}} \mathrm{~d} \xi=\int_{\mathbf{R}^{N}}\left(1+4 \pi^{2}|\xi|^{2}\right)^{\alpha-1}|\widehat{u}|^{2} \mathrm{~d} \xi .
\end{aligned}
$$


On the other hand, recalling $u \in C_{\mathrm{b}}^{1}$, we have

$$
\begin{aligned}
\int_{\mathbf{R}^{N}} u x \cdot \nabla\left(\rho_{\varepsilon} * f(u)\right) \mathrm{d} x & =\sum_{i=1}^{N} \int_{\mathbf{R}^{N}} u x_{i} \partial_{x_{i}}\left(\rho_{\varepsilon} * f(u)\right) \mathrm{d} x=-\sum_{i=1}^{N} \int_{\mathbf{R}^{N}} \partial_{x_{i}}\left(u x_{i}\right) \rho_{\varepsilon} * f(u) \mathrm{d} x \\
& =-N \int_{\mathbf{R}^{N}} u \rho_{\varepsilon} * f(u) \mathrm{d} x-\sum_{i=1}^{N} \int_{\mathbf{R}^{N}} x_{i}\left(\partial_{x_{i}} u\right) \rho_{\varepsilon} * f(u) \mathrm{d} x .
\end{aligned}
$$

From the decay estimate of $u$, the same estimate holds for $\rho_{\varepsilon} * f(u)$ uniformly with respect to $\varepsilon$. Thus, letting $\varepsilon \rightarrow 0$ in the above equality, the dominated convergence theorem and (4) give us

$$
\begin{aligned}
\lim _{\varepsilon \rightarrow 0} \int_{\mathbf{R}^{N}} u x \cdot \nabla\left(\rho_{\varepsilon} * f(u)\right) \mathrm{d} x & =-N \int_{\mathbf{R}^{N}} u f(u) \mathrm{d} x-\sum_{i=1}^{N} \int_{\mathbf{R}^{N}} x_{i}\left(\partial_{x_{i}} u\right) f(u) \mathrm{d} x \\
& =-N\|u\|_{\alpha}^{2}-\sum_{i=1}^{N} \int_{\mathbf{R}^{N}} x_{i} \partial_{x_{i}} F(u) \mathrm{d} x \\
& =-N\|u\|_{\alpha}^{2}+N \int_{\mathbf{R}^{N}} F(u) \mathrm{d} x .
\end{aligned}
$$

Finally, since $\nabla u_{\varepsilon}(x) \rightarrow \nabla u(x)$ in $L_{\text {loc }}^{\infty}\left(\mathbf{R}^{N}\right)$ and $\left(\nabla u_{\varepsilon}\right)$ is bounded in $L^{\infty}\left(\mathbf{R}^{N}\right)$ due to $u \in C_{\mathrm{b}}^{1}\left(\mathbf{R}^{N}\right)$, one sees

$$
\lim _{\varepsilon \rightarrow 0} \int_{\mathbf{R}^{N}} f(u) x \cdot \nabla u_{\varepsilon} \mathrm{d} x=\int_{\mathbf{R}^{N}} f(u) x \cdot \nabla u \mathrm{~d} x=\int_{\mathbf{R}^{N}} x \cdot \nabla F(u) \mathrm{d} x=-N \int_{\mathbf{R}^{N}} F(u) \mathrm{d} x .
$$

Therefore, collecting (37)-(41), we obtain

$$
0=(N-2 \alpha)\|u\|_{\alpha}^{2}-2 N \int_{\mathbf{R}^{N}} F(u) \mathrm{d} x+2 \alpha \int_{\mathbf{R}^{N}}\left(1+4 \pi^{2}|\xi|^{2}\right)^{\alpha-1}|\widehat{u}|^{2} \mathrm{~d} \xi=2 P(u) .
$$

Thus we complete the proof.

Proof of Theorem 1.1. From Propositions 3.2, 3.3, 3.6 and Lemmas 2.5 and 3.4, the only task is to show that there is a positive solution $u_{1}$ of (4) which corresponds to the value $c_{1}$.

First, select $\left(\gamma_{n}\right) \subset \Gamma_{\mathrm{r}}$ so that $\max _{0 \leq t \leq 1} I\left(\gamma_{n}(t)\right) \rightarrow c_{\mathrm{MP}, \mathrm{r}}$. As in the proof of Lemma 3.4, setting $\eta_{n}(t)(x):=$ $\left|\gamma_{n}(t)\right|(x)$, by Lemma A.3, we observe that $I\left(\eta_{n}(t)\right) \leq I\left(\gamma_{n}(t)\right)$ for every $t \in[0,1]$ and $\eta_{n} \in \Gamma_{\mathrm{r}}$. Hence, it follows from Lemma 3.4 that

$$
\max _{0 \leq t \leq 1} \tilde{I}\left(0, \eta_{n}(t)\right) \rightarrow \tilde{c}_{\mathrm{MP}, \mathrm{r}}=c_{1} .
$$

Thus as in the proof of Proposition 3.2, applying the Eklenad's variational principle to $\tilde{I}$ and $\left(\eta_{n}\right)$, and noting $\eta_{n}(t)(x) \geq 0$, we may find $\left(\theta_{n}\right)$ and $\left(u_{n}\right) \subset H_{\mathrm{r}}^{\alpha}\left(\mathbf{R}^{N}\right)$ so that

$$
I\left(u_{n}\right) \rightarrow c_{1}, \quad D_{(\theta, u)} \tilde{I}\left(\theta_{n}, u_{n}\right) \rightarrow 0, \quad \theta_{n} \rightarrow 0, \quad\left(u_{n}\right)_{-}(x):=\max \{0,-u(x)\} \rightarrow 0 \quad \text { in } H^{\alpha}\left(\mathbf{R}^{N}\right) .
$$

Repeating the argument of Proposition 3.2, $u_{n} \rightarrow u_{0}$ strongly in $H^{\alpha}\left(\mathbf{R}^{N}\right), I\left(u_{0}\right)=c_{1}, u_{0} \geq 0, \not \equiv 0$. Using Propositions 3.5 and 3.6, we have $u \in C_{\mathrm{b}}^{\beta}\left(\mathbf{R}^{N}\right)$ for any $\beta \in(0,2 \alpha)$. Finally, a weak Harnack inequality ( [21, Proposition 2]) gives $u_{1,0}>0$ in $\mathbf{R}^{N}$ and we complete the proof.

\section{Proof of Theorem 1.3}

In this section, we shall prove Theorem 1.3 using Theorem 1.1. Until the proof of Theorem 1.3, we consider the more general setting. Indeed, we first assume that $f(x, s)$ in $(1)$ satisfies the following:

(G1) $f \in C\left(\mathbf{R}^{N} \times \mathbf{R}, \mathbf{R}\right)$ and $f(x,-s)=-f(x, s)$ for each $x \in \mathbf{R}^{N}$ and $s \in \mathbf{R}$.

$$
-\infty<\liminf _{s \rightarrow 0} \inf _{x \in \mathbf{R}^{N}} \frac{f(x, s)}{s} \leq \limsup _{s \rightarrow 0} \sup _{x \in \mathbf{R}^{N}} \frac{f(x, s)}{s}<1 .
$$

$$
\lim _{|s| \rightarrow \infty} \sup _{x \in \mathbf{R}^{N}} \frac{|f(x, s)|}{|s|_{\alpha}^{2 *-1}}=0 .
$$

(G4) There exists an $s_{0}>0$ such that

$$
\inf _{x \in \mathbf{R}^{N}}\left(F\left(x, s_{0}\right)-\frac{1}{2} s_{0}^{2}\right)>0 .
$$

(G5) There exists a $f_{\infty}(s) \in C(\mathbf{R}, \mathbf{R})$ such that $f(x, s) \rightarrow f_{\infty}(s)$ in $L_{\mathrm{loc}}^{\infty}(\mathbf{R})$ as $|x| \rightarrow \infty$. 
Note that (G1)-(G3) and (G5) are weaker than (F1)-(F5). Moreover, we remark that (F5) implies (G4). Indeed, by the inequalities in (F5), we have

$$
G(x, s) \geq\left(\frac{s}{s_{1}}\right)^{\mu} G\left(x, s_{1}\right) \geq c_{1} s^{\mu} \quad \text { for all }(x, s) \in \mathbf{R}^{N} \times\left[s_{1}, \infty\right)
$$

for some $c_{1}>0$. Since $\mu>2$ and $V \in L^{\infty}\left(\mathbf{R}^{N}\right)$, we can easily find an $s_{0}>0$ such that

$$
F\left(x, s_{0}\right)-\frac{1}{2} s_{0}^{2}=G\left(x, s_{0}\right)-\frac{V(x)+1}{2} s_{0}^{2} \geq c_{1} s_{0}^{\mu}-\frac{V(x)+1}{2} s_{0}^{2}>0 .
$$

Thus (G4) is derived from (F5).

Under (G1)-(G5), we define the functional $J$ corresponding to (1):

$$
J(u):=\frac{1}{2}\|u\|_{\alpha}^{2}-\int_{\mathbf{R}^{N}} F(x, u(x)) \mathrm{d} x \in C^{1}\left(H^{\alpha}\left(\mathbf{R}^{N}\right), \mathbf{R}\right) .
$$

Remark that a critical point of $J$ is equivalent to a solution of (1). We begin with showing that $J$ has the mountain pass geometry:

Proposition 4.1. Under (G1)-(G5), there exist $\rho_{0}>0$ and $u_{1} \in H^{\alpha}\left(\mathbf{R}^{N}\right)$ such that

$$
\inf _{\|u\|_{\alpha}=\rho_{0}} J(u)>0, \quad J(u) \geq 0 \quad \text { if }\|u\|_{\alpha} \leq \rho_{0}, \quad J\left(u_{1}\right)<0 .
$$

Proof. By (G2) and (G3), we find $\delta_{0}>0$ and $s_{1}>0$ such that

$$
\sup _{x \in \mathbf{R}^{N}} \frac{f(x, s)}{s} \leq 1-2 \delta_{0} \quad \text { for all }|s| \leq s_{1}
$$

Hence,

$$
\sup _{x \in \mathbf{R}^{N}} F(x, s) \leq \frac{1-\delta_{0}}{2} s^{2} \quad \text { for every }|s| \leq s_{1} .
$$

Combining this with (G3), we have

$$
\left(F(x, s)-\frac{1-\delta_{0}}{2} s^{2}\right)_{+} \leq C|s|^{2_{\alpha}^{*}} \quad \text { for each } x \in \mathbf{R}^{N}, s \in \mathbf{R} .
$$

Hence, Sobolev's inequality yields

$$
\begin{aligned}
J(u) & =\frac{1}{2}\|u\|_{\alpha}^{2}-\frac{1-\delta_{0}}{2}\|u\|_{L^{2}}^{2}-\int_{\mathbf{R}^{N}}\left(F(x, s)-\frac{1-\delta_{0}}{2} u^{2}\right) \mathrm{d} x \\
& \geq \frac{\delta_{0}}{2}\|u\|_{\alpha}^{2}-\int_{\mathbf{R}^{N}}\left(F(x, s)-\frac{1-\delta_{0}}{2} u^{2}\right)_{+} \mathrm{d} x \geq \frac{\delta_{0}}{2}\|u\|_{\alpha}^{2}-C\|u\|_{\alpha^{*}}^{2^{\alpha}}
\end{aligned}
$$

Choosing $\rho_{0}>0$ sufficiently small, we have

$$
\inf _{\|u\|_{\alpha}=\rho_{0}} J(u)>0, \quad J(u) \geq 0 \quad \text { if }\|u\|_{\alpha} \leq \rho_{0} .
$$

For the existence of $u_{1}$, let us consider a function defined by

$$
u_{1}(x):= \begin{cases}s_{0} & \text { if }|x| \leq R \\ -s_{0}(|x|-R)+s_{0} & \text { if } R<|x| \leq R+1, \\ 0 & \text { if }|x|>R+1\end{cases}
$$

where $s_{0}>0$ appears in (G4). Notice that $u_{1} \in H^{1}\left(\mathbf{R}^{N}\right)$. By $\|u\|_{\alpha} \leq\|u\|_{H^{1}}$ for all $u \in H^{1}\left(\mathbf{R}^{N}\right)$, we obtain

$$
J\left(u_{1}\right) \leq \frac{1}{2}\left\|u_{1}\right\|_{H^{1}}^{2}-\int_{\mathbf{R}^{N}} F\left(x, u_{1}\right) \mathrm{d} x=\frac{1}{2}\left\|\nabla u_{1}\right\|_{L^{2}}^{2}-\int_{\mathbf{R}^{N}} F\left(x, u_{1}\right)-\frac{1}{2} u_{1}^{2} \mathrm{~d} x .
$$

Since it is easy to check $\left\|\nabla u_{1}\right\|_{L^{2}}^{2}=O\left(R^{N-2}\right)$ and $\int_{\mathbf{R}^{N}} F\left(x, u_{1}\right)-\frac{1}{2} u_{1}^{2} \mathrm{~d} x \geq c R^{N}+O\left(R^{N-1}\right)$ as $R \rightarrow \infty$ for some $c>0$, for sufficiently large $R>0$, one finds that

$$
J\left(u_{1}\right) \leq \frac{1}{2}\left\|\nabla u_{1}\right\|_{L^{2}}^{2}-\int_{\mathbf{R}^{N}} F\left(x, u_{1}\right)-\frac{1}{2} u_{1}^{2} \mathrm{~d} x<0 .
$$

Thus we complete the proof. 
By Proposition 4.1, we define the mountain pass value of $J$ :

$$
d_{\mathrm{MP}}:=\inf _{\gamma \in \Gamma_{J}} \max _{0 \leq t \leq 1} J(\gamma(t))>0, \quad \Gamma_{J}:=\left\{\gamma \in C\left([0,1], H^{\alpha}\left(\mathbf{R}^{N}\right)\right) \mid \gamma(0)=0, J(\gamma(1))<0\right\} .
$$

As in the proof of Theorem 1.1, applying Ekeland's variational principle to $J$ and a sequence of paths $\left(\gamma_{n}(t)\right) \subset \Gamma_{J}$ where $\gamma_{n}(t)(x) \geq 0$ and $\max _{0 \leq t \leq 1} J\left(\gamma_{n}(t)\right) \rightarrow d_{\mathrm{MP}}$, we may find a Palais-Smale sequence $\left(v_{n}\right)$ of $J$ at level $d_{\mathrm{MP}}$ :

$$
J\left(v_{n}\right) \rightarrow d_{\mathrm{MP}}, \quad J\left(v_{n}\right) \rightarrow 0 \quad \text { strongly in }\left(H^{\alpha}\left(\mathbf{R}^{N}\right)\right)^{*}, \quad\left(v_{n}\right)_{-} \rightarrow 0 \quad \text { strongly in } H^{\alpha}\left(\mathbf{R}^{N}\right) .
$$

We first observe the behaviors of bounded Palais-Smale sequneces of $J$ under (G1)-(G5). For this purpose, consider

$$
(1-\Delta)^{\alpha} \omega=f_{\infty}(\omega) \quad \text { in } \mathbf{R}^{N}, \quad \omega \in H^{\alpha}\left(\mathbf{R}^{N}\right)
$$

and define the functional $J_{\infty}$ corresponding to (43) by

$$
J_{\infty}(\omega):=\frac{1}{2}\|\omega\|_{\alpha}^{2}-\int_{\mathbf{R}^{N}} F_{\infty}(\omega) \mathrm{d} x
$$

where $F_{\infty}(s):=\int_{0}^{s} f_{\infty}(t) \mathrm{d} t$. Notice that $f_{\infty}$ satisfies (f1)-(f4) thanks to (G1)-(G5). Hence, $J_{\infty} \in C^{1}\left(H^{\alpha}\left(\mathbf{R}^{N}\right), \mathbf{R}\right)$ and critical points of $J$ are solutions of (43).

Proposition 4.2. Suppose that (G1)-(G5) hold and that every weak solution of (43) satisfies the Pohozaev identity $P_{\infty}(u)=0$ where

$$
P_{\infty}(u):=\frac{N-2 \alpha}{2}\|u\|_{\alpha}^{2}-N \int_{\mathbf{R}^{N}} F_{\infty}(u) \mathrm{d} x+\alpha \int_{\mathbf{R}^{N}}\left(1+4 \pi^{2}|\xi|^{2}\right)^{\alpha-1}|\widehat{u}|^{2} \mathrm{~d} \xi
$$

Let $\left(u_{n}\right)$ be a bounded Palais-Smale sequence of $J$, that is, $\left(u_{n}\right) \subset H^{\alpha}\left(\mathbf{R}^{N}\right)$ is bounded and satisfies

$$
J\left(u_{n}\right) \rightarrow c \in \mathbf{R}, \quad J^{\prime}\left(u_{n}\right) \rightarrow 0 \quad \text { strongly in }\left(H^{\alpha}\left(\mathbf{R}^{N}\right)\right)^{*} .
$$

Then there exist $\ell \in \mathbf{N}, u_{0} \in H^{\alpha}\left(\mathbf{R}^{N}\right), \omega_{i} \in H^{\alpha}\left(\mathbf{R}^{N}\right)$ and $\left(y_{n, i}\right)_{n=1}^{\infty}$ for $i=1, \ldots, \ell$ provided $\ell \geq 1$ such that

(i) $\left|y_{n, i}\right| \rightarrow \infty$ for $1 \leq i \leq \ell$ and $\left|y_{n, i}-y_{n, j}\right| \rightarrow \infty$ for $i \neq j$ as $n \rightarrow \infty$.

(ii) $J^{\prime}\left(u_{0}\right)=0, \omega_{i} \not \equiv 0$ and $J_{\infty}^{\prime}\left(\omega_{i}\right)=0$ for $1 \leq i \leq \ell$.

(iii) When $\ell \geq 1$,

$$
\left\|u_{n}-u_{0}-\sum_{i=1}^{\ell} \omega_{i}\left(\cdot-y_{n, i}\right)\right\|_{\alpha} \rightarrow 0, \quad c=\lim _{n \rightarrow \infty} J\left(u_{n}\right)=I\left(u_{0}\right)+\sum_{i=1}^{\ell} J_{\infty}\left(\omega_{i}\right)
$$

When $\ell=0,\left\|u_{n}-u_{0}\right\|_{\alpha} \rightarrow 0$ and $c=J\left(u_{0}\right)$.

We postpone a proof of Proposition 4.2 and prove it after the proof of Theorem 1.3.

Next, since $f_{\infty}$ satisfies (f1)-(f4) under (G1)-(G5), we may define the mountain pass value of $J_{\infty}$ :

$$
d_{\infty}:=\inf _{\gamma \in \Gamma_{J_{\infty}}} \max _{0 \leq t \leq 1} J_{\infty}(\gamma(t))>0, \quad \Gamma_{J_{\infty}}:=\left\{\gamma \in C\left([0,1], H^{\alpha}\left(\mathbf{R}^{N}\right)\right) \mid \gamma(0)=0, J_{\infty}(\gamma(1))<0\right\} .
$$

Proposition 4.3. Let (G1)-(G5) hold. Furthermore, suppose that

(I) $F_{\infty}(s) \leq F(x, s)$ for all $(x, s) \in \mathbf{R}^{N} \times \mathbf{R}$.

(II) There exists a bounded Palais-Smale sequence $\left(u_{n}\right)$ of $J$ at level $d_{\mathrm{MP}}$ with $\left(u_{n}\right)_{-} \rightarrow 0$ strongly in $H^{\alpha}\left(\mathbf{R}^{N}\right)$.

(III) Every weak solution of (43) satisfies the Pohozaev identity $P_{\infty}(u)=0$.

Then, (1) admits a positive solution.

Proof. By (I), we observe that $J(u) \leq J_{\infty}(u)$ for all $u \in H^{\alpha}\left(\mathbf{R}^{N}\right)$. Hence, $\Gamma_{J_{\infty}} \subset \Gamma_{J}$ and $0<d_{\mathrm{MP}} \leq d_{\infty}$ hold. We divide our case into two cases:

Case 1: $d_{\mathrm{MP}}<d_{\infty}$ holds.

In this case, we apply Proposition 4.2 for $\left(u_{n}\right)$ to obtain $u_{0}, \omega_{1}, \ldots, \omega_{\ell} \in H^{\alpha}\left(\mathbf{R}^{N}\right)$ and $\left(y_{n, i}\right)_{n=1}^{\infty}(i=1, \ldots, \ell)$ satisfying (i)-(iii) in Proposition 4.2. Remark that $J^{\prime}\left(u_{0}\right)=0$ and $u_{0}$ is a weak limit of $\left(u_{n}\right)$ (see the proof of Proposition 4.2 below). Arguing as in the proof of Proposition 3.6, we observe that $u_{0} \in C_{\mathrm{b}}^{\beta}\left(\mathbf{R}^{N}\right)$ and $u_{0}$ is nonnegative. Therefore, if $u_{0} \not \equiv 0$, then a weak Harnack inequality ( $\left[21\right.$, Proposition 2]) implies that $u_{0}$ is the desired solution of (1). Thus, it suffices to show that the case $u_{0} \equiv 0$ does not happen. 
To this end, let $u_{0} \equiv 0$ and we may assume $\ell \geq 1$ thanks to $d_{\mathrm{MP}}>0$. Since $\omega_{i} \not \equiv 0$ and $J_{\infty}^{\prime}\left(\omega_{i}\right)=0$, Theorem 1.1 and the assumption (III) assert that $J_{\infty}\left(\omega_{i}\right) \geq d_{\infty}>0$ for all $1 \leq i \leq \ell$. Thus Proposition 4.2 (iii) and $u_{0} \equiv 0$ yield

$$
d_{\infty}>d_{\mathrm{MP}}=\sum_{i=1}^{\ell} J_{\infty}\left(\omega_{i}\right) \geq \ell d_{\infty}
$$

Since $d_{\infty}>0$, this is a contradiction. Hence, $u_{0} \equiv 0$ does not occur in this case.

Case 2: $d_{\mathrm{MP}}=d_{\infty}$ holds.

In this case, by Theorem 1.1, we find a positive solution $\omega \in H^{\alpha}\left(\mathbf{R}^{N}\right)$ of (43) and a path $\gamma_{\omega} \in \Gamma_{J_{\infty}}$ so that

$$
\begin{aligned}
& \omega(0)=\|\omega\|_{L^{\infty}}, \quad \omega \in \gamma_{\omega}([0,1]), \quad\|\omega\|_{L^{\infty}}=\left\|\gamma_{\omega}(t)\right\|_{L^{\infty}} \text { for } t \in(0,1], \\
& J_{\infty}(\omega)=\max _{0 \leq t \leq 1} J_{\infty}\left(\gamma_{\omega}(t)\right)=d_{\infty}, \quad J_{\infty}\left(\gamma_{\omega}(t)\right)<J_{\infty}(\omega) \quad \text { if } \gamma_{\omega}(t) \neq \omega .
\end{aligned}
$$

Noting $\gamma_{\omega}(t)(\cdot-z) \in \Gamma_{J_{\infty}} \subset \Gamma_{J}$ for any $z \in \mathbf{R}^{N}$, let $t_{z} \in(0,1)$ satisfy $\max _{0 \leq t \leq 1} J\left(\gamma_{\omega}(t)(\cdot-z)\right)=J\left(\gamma_{\omega}\left(t_{z}\right)(\cdot-z)\right)$. Then we get

$$
d_{\infty}=d_{\mathrm{MP}} \leq J\left(\gamma_{\omega}\left(t_{z}\right)(\cdot-z)\right) \leq J_{\infty}\left(\gamma_{\omega}\left(t_{z}\right)(\cdot-z)\right) \leq J_{\infty}(\omega)=d_{\infty} .
$$

From (44) and (45), we deduce that $\gamma_{\omega}\left(t_{z}\right)=\omega$ and

$$
\int_{\mathbf{R}^{N}} F(x, \omega(x-z)) \mathrm{d} x=\int_{\mathbf{R}^{N}} F_{\infty}(\omega(x-z)) \mathrm{d} x \quad \text { for any } z \in \mathbf{R}^{N} .
$$

Recalling $F_{\infty}(s) \leq F(x, s)$ for any $(x, s) \in \mathbf{R}^{N} \times \mathbf{R}$, we observe that

$$
F(x, s)=F_{\infty}(s) \text { for all }(x, s) \in \mathbf{R}^{N} \times\left[0,\|\omega\|_{L^{\infty}}\right] .
$$

This implies $f(x, s)=f_{\infty}(s)$ for all $x \in \mathbf{R}^{N} \times\left[0,\|\omega\|_{L^{\infty}}\right]$. From this fact, we see that $\omega$ is also a positive solution of (1). Thus we complete the proof.

Remark 4.4. (i) From the above proof and the existence of optimal path in Theorem 1.1, we have $d_{\mathrm{MP}}<d_{\infty}$ when $F(x, s) \leq F_{\infty}(s)$ and $F(x, s) \not \equiv F_{\infty}(s)$ for each $s \in \mathbf{R}$.

(ii) In the case $\alpha=1$, the Pohozaev identity is useful to obtain a bounded Palais-Smale sequence. For instance, we refer to $[5,28]$. When $0<\alpha<1$, in addition to $(\mathrm{G} 1)-(\mathrm{G} 4)$, assume that $f(x, s)$ is differentiable in $x, \nabla_{x} f(x, s) \in C\left(\mathbf{R}^{N} \times \mathbf{R}, \mathbf{R}^{N}\right)$ and for each $M>0$, there exists a $C_{M}>0$ such that $\left|\nabla_{x} f(x, s)\right| \leq C_{M}$ for all $(x, s) \in \mathbf{R}^{N} \times[-M, M]$. Under these conditions, if $u \in H^{\alpha}\left(\mathbf{R}^{N}\right) \cap C_{\mathrm{b}}^{1}\left(\mathbf{R}^{N}\right)$ is a weak solution of (1), then $u$ satisfies the following Pohozaev identity:

$$
0=\frac{N-2 \alpha}{2}\|u\|_{\alpha}^{2}-N \int_{\mathbf{R}^{N}} F(x, u) \mathrm{d} x-\int_{\mathbf{R}^{N}}\left(x \cdot \nabla_{x} F\right)(x, u) \mathrm{d} x+\alpha \int_{\mathbf{R}^{N}}\left(1+4 \pi^{2}|\xi|^{2}\right)^{\alpha-1}|\widehat{u}|^{2} \mathrm{~d} \xi .
$$

In fact, (46) can be proved by following the argument of Proposition 3.6 (iii) and noting

$$
\sum_{i=1}^{N} x_{i} \partial_{x_{i}} u f(x, u)=\sum_{i=1}^{N} x_{i}\left\{\partial_{x_{i}}(F(x, u))-\left(\partial_{x_{i}} F(x, u)\right)\right\}=\left(x \cdot \nabla_{x}\right) F(x, u)-\left(x \cdot \nabla_{x} F\right)(x, u) .
$$

As in the case $\alpha=1$, the Pohozaev identity (46) may be useful to get a bounded Palais-Smale sequence.

Now we prove Theorem 1.3.

Proof of Theorem 1.3. Let us assume (F1)-(F5). As we have already seen, (G1)-(G5) also holds. Moreover, conditions (I) and (III) in Proposition 4.3 follow from (F4) and Theorem 1.1. Thus we only need to check that (II) holds. For this purpose, we use (F5) to show that $\left(v_{n}\right)$ in (42) is bounded in $H^{\alpha}\left(\mathbf{R}^{N}\right)$ and the argument is standard (for instance, see [33]). From (F5), we have

$$
\begin{aligned}
\mu d_{\mathrm{MP}}+o(1)+o(1)\left\|v_{n}\right\|_{\alpha} & \geq \mu J\left(v_{n}\right)-J^{\prime}\left(v_{n}\right) v_{n} \\
& =\frac{\mu-2}{2}\left(\left\|v_{n}\right\|_{\alpha}^{2}+\int_{\mathbf{R}^{N}} V(x) v_{n}^{2} \mathrm{~d} x\right)-\int_{\mathbf{R}^{N}} \mu G\left(x, v_{n}\right)-g\left(x, v_{n}\right) v_{n} \mathrm{~d} x \\
& \geq \frac{\mu-2}{2}\left(\left\|v_{n}\right\|_{\alpha}^{2}+\int_{\mathbf{R}^{N}} V(x) v_{n}^{2} \mathrm{~d} x\right) .
\end{aligned}
$$

Since $\inf _{\mathbf{R}^{N}} V>-1$ due to (F2), a quantity defined by

$$
\|u\|^{2}:=\|u\|_{\alpha}^{2}+\int_{\mathbf{R}^{N}} V(x) u^{2} \mathrm{~d} x
$$


is an equivalent norm to $\|\cdot\|_{\alpha}$. Therefore, from (47), we infer that $\left(v_{n}\right)$ is bounded in $H^{\alpha}\left(\mathbf{R}^{N}\right)$ and Proposition 4.3 implies Theorem 1.3.

Now we turn to prove Proposition 4.2. We first recall the following lemma due to [22, Lemma 2.1] (cf. [18, Lemma 2.18], [31, Lemma I.1] and [38, Lemma 3.1]):

Lemma 4.5. Let $\left(u_{n}\right) \subset H^{\alpha}\left(\mathbf{R}^{N}\right)$ be a bounded and satisfy

$$
\sup _{y \in \mathbf{Z}^{N}} \int_{y+Q}\left|u_{n}\right|^{p} \mathrm{~d} x \rightarrow 0 \quad \text { for some } p \in\left[2,2_{\alpha}^{*}\right), \quad Q:=[0,1]^{N} .
$$

Then $u_{n} \rightarrow 0$ strongly in $L^{q}\left(\mathbf{R}^{N}\right)$ for all $q \in\left(2,2_{\alpha}^{*}\right)$.

This lemma is proved in [22, Lemma 2.1], however, for the sake of readers, we show it here.

Proof. First we note that by the boundedness of $\left(u_{n}\right)$ and the interpolation inequality, we may assume $2<p$ without loss of generality. Next, we shall prove the existence of $C_{0}>0$ satisfying

$$
\|u\|_{L^{p}(z+Q)} \leq C_{0}\|u\|_{W^{2, \alpha}(z+Q)} \quad \text { for all } z \in \mathbf{Z}^{N}, u \in W^{2, \alpha}(z+Q)
$$

where

$$
W^{2, \alpha}(\Omega):=\left\{u \in L^{2}(\Omega) \mid[u]_{W^{2, \alpha}(\Omega)}<\infty\right\}, \quad[u]_{W^{2, \alpha}(\Omega)}^{2}:=\int_{\Omega \times \Omega} \frac{|u(x)-u(y)|^{2}}{|x-y|^{N+2 \alpha}} \mathrm{d} x \mathrm{~d} y
$$

We first consider it on $Q$. For $u \in W^{2, \alpha}(Q)$, let

$$
\tilde{u}\left(x^{\prime}, x_{N}\right):= \begin{cases}u\left(x^{\prime}, x_{N}\right) & \text { if } x_{N} \geq 0 \\ u\left(x^{\prime},-x_{N}\right) & \text { if } x_{N}<0 .\end{cases}
$$

Set $Q_{1}:=Q \cup\left(Q-\mathbf{e}_{N}\right)$ and $R x:=\left(x^{\prime},-x_{N}\right)$ where $\mathbf{e}_{N}:=(0, \ldots, 0,1)$. Since $|x-y|=|R x-R y|$ and $|x-y| \leq|R x-y|$ for all $x, y \in Q$, and $\tilde{u}(R x)=u(x)$ for $x \in Q$, it is easy to see that

$$
\begin{aligned}
\|\tilde{u}\|_{L^{2}\left(Q_{1}\right)}^{2} & =2\|u\|_{L^{2}(Q)}^{2}, \\
{[\tilde{u}]_{W^{2, \alpha}\left(Q_{1}\right)}^{2} } & =\int_{Q_{1} \times Q_{1}} \frac{|\tilde{u}(x)-\tilde{u}(y)|^{2}}{|x-y|^{N+2 \alpha}} \mathrm{d} x \mathrm{~d} y \\
& =\left(\int_{Q \times Q}+\int_{Q \times\left(Q-\mathbf{e}_{N}\right)}+\int_{\left(Q-\mathbf{e}_{N}\right) \times Q}+\int_{\left(Q-\mathbf{e}_{N}\right) \times\left(Q-\mathbf{e}_{N}\right)}\right) \frac{|\tilde{u}(x)-\tilde{u}(y)|^{2}}{|x-y|^{N+2 \alpha}} \mathrm{d} x \mathrm{~d} y \\
& \leq 4 \int_{Q \times Q} \frac{|u(x)-u(y)|^{2}}{|x-y|^{N+2 \alpha}}=4[u]_{W^{2, \alpha}(Q)}^{2} .
\end{aligned}
$$

Thus $\tilde{u} \in W^{2, \alpha}\left(Q_{1}\right)$ and $\|\tilde{u}\|_{W^{2, \alpha}\left(Q_{1}\right)} \leq 2\|u\|_{W^{2, \alpha}(Q)}$.

Repeating the above argument $2 N-1$ times for edges of $Q_{1}$ except for $x_{N}=-1$, there exists an Eu $\in$ $W^{2, \alpha}\left(Q_{2 N}\right)$ such that

$$
E u=u \quad \text { on } Q, \quad\|E u\|_{W^{2, \alpha}\left(Q_{2 N}\right)} \leq 2^{2 N}\|u\|_{W^{2, \alpha}(Q)}
$$

where $Q_{2 N}$ is a cube satisfying $[-1,2]^{N} \subset Q_{2 N}$. Choosing a smooth domain $\Omega \subset \mathbf{R}^{N}$ such that $Q \subset \Omega \subset[-1,2]^{N}$, it follows from Sobolev's embedding ( [20, Theorems 5.6 and 6.7]) that

$$
\|u\|_{L^{p}(Q)} \leq\|E u\|_{L^{p}(\Omega)} \leq C_{\Omega}\|E u\|_{W^{2, \alpha}(\Omega)} \leq C_{\Omega}\|E u\|_{W^{2, \alpha}\left(Q_{2 N}\right)} \leq 2^{2 N} C_{\Omega}\|u\|_{W^{2, \alpha}(Q)}
$$

for all $u \in W^{2, \alpha}(Q)$. For (48), it is enough to translate $Q, Q_{2 N}$ and $\Omega$. Thus (48) holds.

Now we complete the proof. We first notice from [20, Proposition 3.4] that

$$
[u]_{W^{2, \alpha}\left(\mathbf{R}^{N}\right)}^{2}=C(N, \alpha) \int_{\mathbf{R}^{N}}|\xi|^{2 \alpha}|\hat{u}(\xi)|^{2} \mathrm{~d} \xi .
$$

Thus, it is easily seen that

$$
\begin{aligned}
\sum_{z \in \mathbf{Z}^{N}}[u]_{W^{2, \alpha}(z+Q)}^{2} & =\sum_{z \in \mathbf{Z}^{N}} \int_{(z+Q) \times(z+Q)} \frac{|u(x)-u(y)|^{2}}{|x-y|^{N+2 \alpha}} \mathrm{d} x \mathrm{~d} y \\
& \leq \sum_{z \in \mathbf{Z}^{N}} \int_{\mathbf{R}^{N} \times(z+Q)} \frac{|u(x)-u(y)|^{2}}{|x-y|^{N+2 \alpha}} \mathrm{d} x \mathrm{~d} y=[u]_{W^{2, \alpha}\left(\mathbf{R}^{N}\right)}^{2} \leq C\|u\|_{\alpha}^{2} .
\end{aligned}
$$


Therefore, by (48) and $p>2$, we obtain

$$
\begin{aligned}
\left\|u_{n}\right\|_{L^{p}\left(\mathbf{R}^{N}\right)}^{p} & =\sum_{z \in \mathbf{Z}^{N}}\left\|u_{n}\right\|_{L^{p}(z+Q)}^{p} \leq\left(\sup _{z \in \mathbf{Z}^{N}}\left\|u_{n}\right\|_{L^{p}(z+Q)}^{p-2}\right) \sum_{z \in \mathbf{Z}^{N}}\left\|u_{n}\right\|_{L^{p}(z+Q)}^{2} \\
& \leq\left(\sup _{z \in \mathbf{Z}^{N}}\left\|u_{n}\right\|_{L^{p}(z+Q)}^{p-2}\right) \sum_{z \in \mathbf{Z}^{N}} C_{0}\|u\|_{W^{2, \alpha}(z+Q)}^{2} \\
& \leq C_{0}\left(\sup _{z \in \mathbf{Z}^{N}}\left\|u_{n}\right\|_{L^{p}(z+Q)}^{p-2}\right)\left\|u_{n}\right\|_{\alpha}^{2} \rightarrow 0 .
\end{aligned}
$$

Now we prove Proposition 4.2.

Proof of Proposition 4.2. We argue as in [27, Proof of Proposition 4.2]. Since $\left(u_{n}\right)$ is bounded in $H^{\alpha}\left(\mathbf{R}^{N}\right)$, choosing a subsequence if necessary (still denoted by $\left(u_{n}\right)$ ), we may assume $u_{n} \rightarrow u_{0}$ weakly in $H^{\alpha}\left(\mathbf{R}^{N}\right)$. From $J^{\prime}\left(u_{n}\right) \rightarrow 0$ strongly in $\left(H^{\alpha}\left(\mathbf{R}^{N}\right)\right)^{*}$, it is easy to check $J^{\prime}\left(u_{0}\right)=0$.

Next, we claim that there exist $\ell \geq 0, \omega_{i} \not \equiv 0$ and $\left(y_{n, i}\right)_{n=1}^{\infty}$ for $i=1, \ldots, \ell$ if $\ell \neq 0$ such that properties (i) and (ii) in Proposition 4.2 hold and

$$
\left\|u_{n}-u_{0}-\sum_{i=1}^{\ell} \omega_{i}\left(\cdot-y_{n, i}\right)\right\|_{L^{p}} \rightarrow 0 \quad \text { for every } p \in\left(2,2_{\alpha}^{*}\right) .
$$

For this purpose, we consider

$$
\limsup _{n \rightarrow \infty} \sup _{z \in \mathbf{Z}^{N}} \int_{z+Q}\left|u_{n}-u_{0}\right|^{2} \mathrm{~d} x=: c_{1}
$$

If $c_{1}=0$, then Lemma 4.5 yields (49) with $\ell=0$.

Next, consider the case $c_{1}>0$. Then we choose $\left(y_{n, 1}\right) \subset \mathbf{R}^{N}$ such that

$$
\lim _{n \rightarrow \infty} \int_{y_{n, 1}+Q}\left|u_{n}-u_{0}\right|^{2} \mathrm{~d} x \rightarrow c_{1}>0 .
$$

Let $u_{n}\left(\cdot+y_{n, 1}\right) \rightarrow \omega_{1}$ weakly in $H^{\alpha}\left(\mathbf{R}^{N}\right)$. Since $u_{n} \rightarrow u_{0}$ strongly in $L_{\text {loc }}^{2}\left(\mathbf{R}^{N}\right)$ and $c_{1}>0$, we have $\left|y_{n, 1}\right| \rightarrow \infty$ and $\omega_{1} \not \equiv 0$. Moreover, from $\left|y_{n, 1}\right| \rightarrow \infty$ and $J^{\prime}\left(u_{n}\right)\left[\varphi\left(\cdot-y_{n, 1}\right)\right] \rightarrow 0$ for each $\varphi \in C_{0}^{\infty}\left(\mathbf{R}^{N}\right)$, we also see that $J_{\infty}^{\prime}\left(\omega_{1}\right)=0$ by (G5). Since every weak solution of (43) satisfies the Pohozaev identity $P_{\infty}(u)=0$ and $f_{\infty}$ satisfies (f1)-(f4), by Theorem 1.1, we have $J_{\infty}\left(\omega_{1}\right) \geq d_{\infty}>0$. Choosing a $\zeta_{0}>0$ so that $J_{\infty}(u)<d_{\infty}$ for all $\|u\|_{\alpha}<\zeta_{0}$, we obtain $\left\|\omega_{1}\right\|_{\alpha} \geq \zeta_{0}$.

Next, consider

$$
\limsup _{n \rightarrow \infty} \sup _{z \in \mathbf{Z}^{N}} \int_{z+Q}\left|u_{n}-u_{0}-\omega_{1}\left(x-y_{n, 1}\right)\right|^{2} \mathrm{~d} x=: c_{2}
$$

When $c_{2}=0$, then Lemma 4.5 yields (49). On the other hand, when $c_{2}>0$, we select a $\left(y_{n, 2}\right) \subset \mathbf{R}^{N}$ so that

$$
\lim _{n \rightarrow \infty} \int_{y_{n, 2}+Q}\left|u_{n}-u_{0}-\omega_{1}\left(x-y_{n, 1}\right)\right|^{2} \mathrm{~d} x=c_{2} .
$$

Let $u_{n, 2}\left(x+y_{n, 2}\right)-u_{0}\left(x+y_{n, 2}\right)-\omega_{1}\left(x-y_{n, 1}+y_{n, 2}\right) \rightarrow \omega_{2}$ weakly in $H^{\alpha}\left(\mathbf{R}^{N}\right)$. Then as in the above, it is immediate to see that

$$
\left|y_{n, 2}\right| \rightarrow \infty,\left|y_{n, 1}-y_{n, 2}\right| \rightarrow \infty, u_{n}\left(x+y_{n, 2}\right) \rightarrow \omega_{2} \not \equiv 0, J_{\infty}^{\prime}\left(\omega_{2}\right)=0, J_{\infty}\left(\omega_{2}\right) \geq d_{\infty},\left\|\omega_{2}\right\|_{\alpha} \geq \zeta_{0} .
$$

Now we repeat the same procedure. Namely, consider

$$
\limsup _{n \rightarrow \infty} \sup _{z \in \mathbf{Z}^{N}} \int_{z+Q}\left|u_{n}-u_{0}-\omega_{1}\left(x-y_{n, 1}\right)-\omega_{2}\left(x-y_{n, 2}\right)\right|^{2} \mathrm{~d} x=: c_{3}
$$

and find $\omega_{3} \not \equiv 0$ and $\left(y_{n, 3}\right)$ if $c_{3}>0$. Therefore, we obtain $\ell \in \mathbf{N}, \omega_{i} \not \equiv 0(1 \leq i \leq \ell)$ and $\left(y_{n, i}\right)_{n=1}^{\infty}(1 \leq i \leq \ell)$ so that

$$
\begin{aligned}
& \left|y_{n, i}\right| \rightarrow \infty,\left|y_{n, i}-y_{n, j}\right| \rightarrow \infty \text { if } i \neq j, u_{n}\left(x+y_{n, i}\right) \rightarrow \omega_{i} \not \equiv 0, \\
& J_{\infty}^{\prime}\left(\omega_{i}\right)=0, \quad J_{\infty}\left(\omega_{i}\right) \geq d_{\infty},\left\|\omega_{i}\right\|_{\alpha} \geq \zeta_{0} .
\end{aligned}
$$

To prove (49), it suffices to prove that this procedure cannot be iterated infinitely many times and

$$
\limsup _{n \rightarrow \infty} \sup _{z \in \mathbf{Z}^{N}} \int_{z+Q}\left|u_{n}-u_{0}-\sum_{i=1}^{\ell} \omega_{i}\left(x-y_{n, i}\right)\right|^{2} \mathrm{~d} x=0 \quad \text { for some } \ell \in \mathbf{N} \text {. }
$$


To see this, from (50) it follows that

$$
\begin{aligned}
0 \leq & \left\|u_{n}-u_{0}-\sum_{i=1}^{\ell} \omega_{i}\left(\cdot-y_{n, i}\right)\right\|_{\alpha}^{2}=\left\|u_{n}\right\|_{\alpha}^{2}+\left\|u_{0}\right\|_{\alpha}^{2}+\sum_{i=1}^{\ell}\left\|\omega_{i}\right\|_{\alpha}^{2} \\
& -2\left\langle u_{n}, u_{0}\right\rangle_{\alpha}-2 \sum_{i=1}^{\ell}\left\langle u_{n}, \omega_{i}\left(\cdot-y_{n, i}\right)\right\rangle_{\alpha} \\
& +2 \sum_{i=1}^{\ell}\left\langle u_{0}, \omega_{i}\left(\cdot-y_{n, i}\right)\right\rangle_{\alpha}+2 \sum_{1 \leq i<j \leq \ell}\left\langle\omega_{i}\left(\cdot-y_{n, i}\right), \omega_{j}\left(\cdot-y_{n, j}\right)\right\rangle_{\alpha} \\
= & \left\|u_{n}\right\|_{\alpha}^{2}-\left\|u_{0}\right\|_{\alpha}^{2}-\sum_{i=1}^{\ell}\left\|\omega_{i}\right\|_{\alpha}^{2}+o(1) \\
\leq & \left\|u_{n}\right\|_{\alpha}^{2}-\left\|u_{0}\right\|_{\alpha}^{2}-\ell \zeta_{0}+o(1) .
\end{aligned}
$$

Since $\left(u_{n}\right)$ is bounded, we observe that the above procedure cannot be iterated infinitely many times. Therefore, (51) holds, which implies (49).

Finally, we shall prove that

$$
\left\|u_{n}-u_{0}-\sum_{i=1}^{\ell} \omega_{i}\left(\cdot-y_{n, i}\right)\right\|_{\alpha} \rightarrow 0 .
$$

To do this, set $U_{n}(x):=u_{n}(x)-u_{0}(x)-\sum_{i=1}^{\ell} \omega_{i}\left(x-y_{n, i}\right)$. By (G2), select $\delta_{0}>0$ and $s_{1}>0$ so that

$$
f(x, s) s \leq\left(1-\delta_{0}\right) s^{2} \text { for all }(x, s) \in \mathbf{R}^{N} \times\left[-s_{1}, s_{1}\right] .
$$

It is clear that a norm

$$
\|u\|^{2}:=\|u\|_{\alpha}^{2}-\left(1-\delta_{0}\right)\|u\|_{L^{2}}^{2}
$$

is equivalent to $\|\cdot\|_{\alpha}$. Therefore, instead of (52), we shall show $\left\|U_{n}\right\| \rightarrow 0$.

To this end, putting $f_{\delta_{0}}(x, s):=f(x, s)-\left(1-\delta_{0}\right) s$ and $f_{\infty, \delta_{0}}(s):=f_{\infty}(s)-\left(1-\delta_{0}\right) s$, we first notice from $J^{\prime}\left(u_{n}\right) \rightarrow 0, J^{\prime}\left(u_{0}\right)=0$ and $J_{\infty}^{\prime}\left(\omega_{i}\right)=0$ that

$$
\begin{aligned}
\left\|U_{n}\right\|^{2}= & \left\|U_{n}\right\|_{\alpha}^{2}-\left(1-\delta_{0}\right)\left\|U_{n}\right\|_{L^{2}}^{2} \\
= & \left\langle u_{n}-u_{0}-\sum_{i=1}^{\ell} \omega_{i}\left(\cdot-y_{n, i}\right), U_{n}\right\rangle_{\alpha}-\left(1-\delta_{0}\right)\left\langle u_{n}-u_{0}-\sum_{i=1}^{\ell} \omega_{i}\left(\cdot-y_{n, i}\right), U_{n}\right\rangle_{L^{2}} \\
= & \int_{\mathbf{R}^{N}} f_{\delta_{0}}\left(x, u_{n}\right) U_{n} \mathrm{~d} x-\int_{\mathbf{R}^{N}} f_{\delta_{0}}\left(x, u_{0}\right) U_{n} \mathrm{~d} x-\sum_{i=1}^{\ell} \int_{\mathbf{R}^{N}} f_{\infty, \delta_{0}}\left(\omega_{i}\left(x-y_{n, i}\right)\right) U_{n} \mathrm{~d} x+o(1) \\
= & \int_{\mathbf{R}^{N}}\left\{f_{\delta_{0}}\left(x, u_{n}\right)-f_{\delta_{0}}\left(x, u_{0}\right)-\sum_{i=1}^{\ell} f_{\delta_{0}}\left(x, \omega_{i}\left(x-y_{n, i}\right)\right)\right\} U_{n} \mathrm{~d} x \\
& \quad+\sum_{i=1}^{\ell} \int_{\mathbf{R}^{N}}\left\{f\left(x, \omega_{i}\left(x-y_{n, i}\right)\right)-f_{\infty}\left(\omega_{i}\left(x-y_{n, i}\right)\right)\right\} U_{n} \mathrm{~d} x+o(1) \\
= & : I_{n}+I I_{n}+o(1) .
\end{aligned}
$$

We shall show $I_{n}=o(1)=I I_{n}$. We first consider $I_{n}$. For any $M>0$, by Hölder's inequality, we have

$$
\begin{aligned}
& \int_{\left[\left|U_{n}\right| \geq M\right]} \mid f_{\delta_{0}}\left(x, u_{n}\right)-f_{\delta_{0}}\left(x, u_{0}\right)-\sum_{i=1}^{\ell} f_{\delta_{0}}\left(x, \omega_{i}\left(x-y_{i, n}\right)\right)|| U_{n} \mid \mathrm{d} x \\
& \leq\left\|U_{n}\right\|_{L^{2 *}\left(\left[\left|U_{n}\right| \geq M\right]\right)}\left(\left\|f_{\delta_{0}}\left(x, u_{n}\right)\right\|_{L^{p^{*}}\left(\left[\left|U_{n}\right| \geq M\right]\right)}+\left\|f_{\delta_{0}}\left(x, u_{0}\right)\right\|_{L^{p^{*}}\left(\left[\left|U_{n}\right| \geq M\right]\right)}\right. \\
&\left.+\sum_{i=1}^{\ell}\left\|f_{\delta_{0}}\left(x, \omega_{i}\left(x-y_{n, i}\right)\right)\right\|_{L^{p^{*}}\left(\left[\left|U_{n}\right| \geq M\right]\right)}\right)
\end{aligned}
$$

where $p^{*}:=2_{\alpha}^{*} /\left(2_{\alpha}^{*}-1\right)$. Since $\left(U_{n}\right)$ is bounded in $L^{2_{\alpha}^{*}}\left(\mathbf{R}^{N}\right)$ and $p^{*}<2$, we have

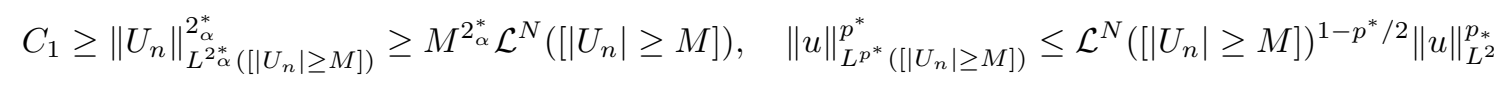


where $C_{1}>0$ is independent of $n$. In particular, $\sup _{n \geq 1} \mathcal{L}^{N}\left(\left[\left|U_{n}\right| \geq M\right]\right) \rightarrow 0$ as $M \rightarrow \infty$. Recalling $\left|f_{\delta_{0}}(x, s)\right| \leq$ $c_{\varepsilon}|s|+\varepsilon|s|^{2_{\alpha}^{*}}$ for all $(x, s) \in \mathbf{R}^{N} \times \mathbf{R}$, it follows from Hölder's inequality and the boundedness of $\left(u_{n}\right)$ that

$$
\begin{aligned}
& \quad \sup _{n \geq 1}\left\{\left\|f_{\delta_{0}}\left(x, u_{n}\right)\right\|_{L^{p^{*}}\left(\left[\left|U_{n}\right| \geq M\right]\right)}^{p^{*}}+\left\|f_{\delta_{0}}\left(x, u_{0}\right)\right\|_{L^{p^{*}}\left(\left[\left|U_{n}\right| \geq M\right]\right)}^{p^{*}}+\left\|f_{\delta_{0}}\left(x, \omega_{i}\left(x-y_{i, n}\right)\right)\right\|_{L^{p^{*}}\left(\left[\left|U_{n}\right| \geq M\right]\right)}^{p^{*}}\right\} \\
& \leq \sup _{n \geq 1} \int_{\left[\left|U_{n}\right| \geq M\right]}\left\{c_{\varepsilon}\left|u_{n}\right|^{p^{*}}+\varepsilon\left|u_{n}\right|^{2_{\alpha}^{*}}+c_{\varepsilon}\left|u_{0}\right|^{p^{*}}+\varepsilon\left|u_{0}\right|^{2_{\alpha}^{*}}\right. \\
& \left.\quad+\sum_{i=1}^{\ell}\left(c_{\varepsilon}\left|\omega_{i}\left(x-y_{n, i}\right)\right|^{p^{*}}+\varepsilon\left|\omega_{i}\left(x-y_{n, i}\right)\right|^{2_{\alpha}^{*}}\right)\right\} \mathrm{d} x \\
& \leq c_{\varepsilon} \mathcal{L}^{N}\left(\left[\left|U_{n}\right| \geq M\right]\right)^{1-p^{*} / 2}+C_{2} \varepsilon
\end{aligned}
$$

for some $C_{2}>0$. Therefore, by (55) and $\sup _{n \geq 1} \mathcal{L}^{N}\left(\left[\left|U_{n}\right| \geq M\right]\right) \rightarrow 0$ as $M \rightarrow \infty$, we get

$$
\limsup _{M \rightarrow \infty} \sup _{n \geq 1} \int_{\left[\left|U_{n}\right| \geq M\right]}\left|f_{\delta_{0}}\left(x, u_{n}\right)-f_{\delta_{0}}\left(x, u_{0}\right)-\sum_{i=1}^{\ell} f_{\delta_{0}}\left(x, \omega_{i}\left(x-y_{i, n}\right)\right)\right|\left|U_{n}\right| \mathrm{d} x \leq C_{2} \varepsilon .
$$

Since $\varepsilon>0$ is arbitrary, we deduce that

$$
\limsup _{M \rightarrow \infty} \sup _{n \geq 1} \int_{\left[\left|U_{n}\right| \geq M\right]}\left|f_{\delta_{0}}\left(x, u_{n}\right)-f_{\delta_{0}}\left(x, u_{0}\right)-\sum_{i=1}^{\ell} f_{\delta_{0}}\left(x, \omega_{i}\left(x-y_{i, n}\right)\right)\right|\left|U_{n}\right| \mathrm{d} x=0 .
$$

On the other hand, denote by $\chi_{n}^{M}(x):=\chi_{\left[\left|U_{n}\right| \leq M\right]}(x)$ the characteristic function of $\left[\left|U_{n}\right| \leq M\right]$. Since $u_{n} \rightarrow u_{0}$ and $u_{n}\left(x+y_{n, i}\right) \rightarrow \omega_{i}$ strongly in $L_{\text {loc }}^{p}\left(\mathbf{R}^{N}\right)$ for every $p<2_{\alpha}^{*}$, for all $R>0$, Strauss' lemma, (G2), (G3), (G5) and the facts $\left|y_{n, i}\right| \rightarrow \infty$ and $\left|y_{n, i}-y_{n, j}\right| \rightarrow \infty$ for $i \neq j$ yield

$$
\begin{aligned}
& \int_{B_{R}(0)} \chi_{n}^{M}(x)\left\{\left|f_{\delta_{0}}\left(x, u_{n}\right)-f_{\delta_{0}}\left(x, u_{0}\right)\right|+\sum_{i=1}^{\ell}\left|f_{\delta_{0}}\left(x, \omega_{i}\left(x-y_{n, i}\right)\right)\right|\right\}\left|U_{n}\right| \mathrm{d} x \\
& \leq M \int_{B_{R}(0)}\left|f_{\delta_{0}}\left(x, u_{n}\right)-f_{\delta_{0}}\left(x, u_{0}\right)\right|+\sum_{i=1}^{\ell}\left|f_{\delta_{0}}\left(x, \omega_{i}\left(x-y_{n, i}\right)\right)\right| \mathrm{d} x \rightarrow 0 \text { as } n \rightarrow \infty, \\
& \int_{B_{R}\left(y_{n, i}\right)} \chi_{n}^{M}(x)\left\{\left|f_{\delta_{0}}\left(x, u_{0}\right)\right|+\sum_{j \neq i}\left|f_{\delta_{0}}\left(x, \omega_{j}\left(x-y_{n, j}\right)\right)\right|\right\}\left|U_{n}\right| \mathrm{d} x \\
& \quad+\int_{B_{R}\left(y_{n, i}\right)} \chi_{n}^{M}(x)\left|f_{\delta_{0}}\left(x, u_{n}\right)-f_{\delta_{0}}\left(x, \omega_{i}\left(x-y_{n, i}\right)\right)\right|\left|U_{n}\right| \mathrm{d} x \\
& \quad \int_{B_{R}(0)}\left\{\mid f_{\delta_{0}}\left(x+y_{n, i}, u_{0}\left(x+y_{n, i}\right)\left|+\sum_{j \neq i}\right| f_{\delta_{0}}\left(x+y_{n, i}, \omega_{j}\left(x+y_{n, i}-y_{n, j}\right)\right) \mid\right.\right. \\
& \left.+\left|f_{\delta_{0}}\left(x+y_{n, i}, u_{n}\left(x+y_{n, i}\right)\right)-f_{\delta_{0}}\left(x+y_{n, i}, \omega_{i}(x)\right)\right|\right\} \mathrm{d} x \rightarrow 0 \quad \text { as } n \rightarrow \infty .
\end{aligned}
$$

Writing $V_{R}:=\mathbf{R}^{N} \backslash\left(B_{R}(0) \cup \bigcup_{i=1}^{\ell} B_{R}\left(y_{n, i}\right)\right)$ and recalling $\left|f_{\delta_{0}}(x, s)\right| \leq c_{1}\left(|s|+|s|^{2_{\alpha}^{*}}\right)$, we have

$$
\begin{aligned}
\int_{V_{R}} \chi_{n}^{M}\left|f_{\delta_{0}}\left(x, u_{0}\right) U_{n}\right| \mathrm{d} x & \leq c_{1} \int_{V_{R}}\left(\left|u_{0}\right|+\left|u_{0}\right|^{2_{\alpha}^{*}-1}\right)\left|U_{n}\right| \mathrm{d} x \\
& \leq c_{1}\left(\left\|u_{0}\right\|_{L^{2}\left(V_{R}\right)}\left\|U_{n}\right\|_{L^{2}\left(V_{R}\right)}+\left\|u_{0}\right\|_{\left.L_{\alpha}^{2 *} V_{R}^{*}\right)}^{2^{*}-1}\left\|U_{n}\right\|_{L^{2 *}}\right)=o_{R}(1)
\end{aligned}
$$

where $o_{R}(1) \rightarrow 0$ as $R \rightarrow 0$ uniformly in $n$ and $M \geq 1$. Similarly, we also obtain

$$
\int_{V_{R}} \chi_{n}^{M}\left|f_{\delta_{0}}\left(x, \omega_{i}\left(x-y_{n, i}\right)\right)\right|\left|U_{n}\right| \mathrm{d} x+\int_{V_{R}} \chi_{n}^{M}\left|f_{\delta_{0}}\left(x, u_{n}\right)\right|\left(\left|u_{0}\right|+\sum_{i=1}^{\ell}\left|\omega_{i}\left(x-y_{n, i}\right)\right|\right) \mathrm{d} x=o_{R}(1) .
$$

Finally, noting that $f_{\delta_{0}}(x, s) s \leq 0$ for all $|s| \leq s_{1}$ and that $\left|f_{\delta_{0}}(x, s) s\right| \leq \varepsilon|s|^{2_{\alpha}^{*}}+c_{\varepsilon}|s|^{p_{0}}$ for all $x \in \mathbf{R}^{N}$ and $|s| \geq s_{1}$ where $p_{0} \in\left(2,2_{\alpha}^{*}\right)$, by $(53)$, we have

$$
\begin{aligned}
\int_{V_{R}} f_{\delta_{0}}\left(x, u_{n}\right) \chi_{n}^{M} u_{n} \mathrm{~d} x & =\int_{V_{R}} f_{\delta_{0}}\left(x, \chi_{n}^{M} u_{n}\right) \chi_{n}^{M} u_{n} \mathrm{~d} x \leq \int_{V_{R} \cap\left[\left|\chi_{n}^{M} u_{n}\right| \geq s_{1}\right]} f_{\delta_{0}}\left(x, \chi_{n}^{M} u_{n}\right) \chi_{n}^{M} u_{n} \mathrm{~d} x \\
& \leq \varepsilon\left\|\chi_{n}^{M} u_{n}\right\|_{L^{2 *}\left(V_{R}\right)}^{2_{\alpha}^{*}}+c_{\varepsilon}\left\|\chi_{n}^{M} u_{n}\right\|_{L^{p_{0}}\left(V_{R}\right)}^{p_{0}} .
\end{aligned}
$$


Recalling (49), $2<p_{0}<2_{\alpha}^{*}$ and the definition of $V_{R}$, we obtain

$$
\begin{aligned}
& \limsup _{n \rightarrow \infty}\left\|\chi_{n}^{M} u_{n}\right\|_{L^{p_{0}}\left(V_{R}\right)} \\
\leq & \limsup _{n \rightarrow \infty}\left(\left\|u_{n}-u_{0}-\sum_{i=1}^{\ell} \omega_{i}\left(\cdot-y_{n, i}\right)\right\|_{L^{p_{0}\left(V_{R}\right)}}+\left\|u_{0}\right\|_{L^{p_{0}\left(V_{R}\right)}}+\sum_{i=1}^{\ell}\left\|\omega_{i}\left(\cdot-y_{n, i}\right)\right\|_{L^{p_{0}\left(V_{R}\right)}}\right) \\
= & o_{R}(1),
\end{aligned}
$$

which implies $\lim \sup _{n \rightarrow \infty}\left|\int_{V_{R}} f_{\delta_{0}}\left(x, u_{n}\right) \chi_{n}^{M} u_{n} \mathrm{~d} x\right| \leq c \varepsilon+c_{\varepsilon} o_{R}(1)$ for some $c>0$. Thus,

$$
\limsup _{R \rightarrow \infty} \limsup _{n \rightarrow \infty}\left|\int_{V_{R}} f_{\delta_{0}}\left(x, u_{n}\right) \chi_{n}^{M} u_{n} \mathrm{~d} x\right| \leq c \varepsilon .
$$

Collecting (58), (59) and (60) with $U_{n}=u_{n}-u_{0}-\sum_{i=1}^{\ell} \omega_{i}\left(x-y_{n, i}\right)$, we observe that

$$
\limsup _{R \rightarrow \infty} \limsup _{n \rightarrow \infty}\left|\int_{V_{R}}\left\{f_{\delta_{0}}\left(x, u_{n}\right)-f_{\delta_{0}}\left(x, u_{0}\right)-\sum_{i=1}^{\ell} f_{\delta_{0}}\left(x, \omega_{i}\left(x-y_{n, i}\right)\right)\right\} \chi_{n}^{M} U_{n} \mathrm{~d} x\right| \leq c \varepsilon .
$$

Since $\varepsilon>0$ is arbitrary, by (57) and (61), we obtain

$$
\limsup _{n \rightarrow \infty}\left|\int_{\mathbf{R}^{N}}\left\{f_{\delta_{0}}\left(x, u_{n}\right)-f_{\delta_{0}}\left(x, u_{0}\right)-\sum_{i=1}^{\ell} f_{\delta_{0}}\left(x, \omega_{i}\left(x-y_{n, i}\right)\right)\right\} \chi_{n}^{M} U_{n} \mathrm{~d} x\right|=0 .
$$

Combining this with (56), we observe that

$$
\limsup _{n \rightarrow \infty}\left|I_{n}\right|=0
$$

In a similar way, we can also prove that $\lim _{\sup _{n \rightarrow \infty}}\left|I I_{n}\right|=0$. Hence, by (54), we get $\left\|U_{n}\right\| \rightarrow 0$ as $n \rightarrow \infty$ and this completes the proof.

\section{Appendix A. Some technical Lemmas}

Here we prove some technical results. First, we show the following:

Proposition A.1. (i) Let $0<\delta_{0}<1$ and define $m(\xi)$ by

$$
m(\xi):=\frac{1}{\left(1+4 \pi^{2}|\xi|^{2}\right)^{\alpha}-\left(1-\delta_{0}\right)} .
$$

Set $K(x):=\mathscr{F}^{-1} m$. Then $K(x) \in C^{\infty}\left(\mathbf{R}^{N} \backslash\{0\}\right)$ and for any $k>0$ there exists a $c_{k}>0$ such that $|K(x)| \leq$ $c_{k}\left(\chi_{B_{1}(0)}(x)|x|^{-N+2 \alpha}+\chi_{B_{1}(0)^{c}}(x)|x|^{-k}\right)$ for all $x \in \mathbf{R}^{N}$.

(ii) For any $g \in L^{2}\left(\mathbf{R}^{N}\right)$, the equation

$$
(1-\Delta)^{\alpha} v-\left(1-\delta_{0}\right) v=g(x) \text { in } \mathbf{R}^{N}
$$

has a unique solution $v \in H^{\alpha}\left(\mathbf{R}^{N}\right)$. Moreover, when $g \in L^{2}\left(\mathbf{R}^{N}\right) \cap L^{\infty}\left(\mathbf{R}^{N}\right)$ and $g(x) \geq 0, \not \equiv 0$, then $v \in C_{\mathrm{b}}^{\beta}\left(\mathbf{R}^{N}\right)$ for any $0<\beta<2 \alpha$ and $v>0$ in $\mathbf{R}^{N}$. In addition, if $\operatorname{supp} g$ is compact and $g \in L^{\infty}\left(\mathbf{R}^{N}\right)$ with $g(x) \geq 0$, then for any $k \in \mathbf{R}^{N}$ one finds a $c_{k}>0$ such that $v(x) \leq c_{k}(1+|x|)^{-k}$ for all $x \in \mathbf{R}^{N}$.

(iii) Suppose that $g_{i} \in L^{2}\left(\mathbf{R}^{N}\right) \cap L^{\infty}\left(\mathbf{R}^{N}\right)(i=1,2)$ satisfy $g_{1} \leq g_{2}$. Let $v_{i} \in H^{\alpha}\left(\mathbf{R}^{N}\right)$ be a unique solution of (62) with $g(x)=g_{i}(x)$. Then $v_{1}(x) \leq v_{2}(x)$ for $x \in \mathbf{R}^{N}$.

Proof. (i) The smoothness of $K$ and the inequality $K(x) \leq C_{0}|x|^{-N+2 \alpha}$ for $|x| \leq 1$ follow from the arguments in $\left[36, \S 4.4\right.$ of Chapter VI]. For the decay estimate at infinity, since $\left(\Delta_{\xi}\right)^{k} m(\xi) \in L^{1}\left(\mathbf{R}^{N}\right)$ provided $k>N / 2$, we have $\left(4 \pi^{2}|x|^{2}\right)^{k} K(x)=\mathscr{F}^{-1}\left(\Delta^{k} m\right) \in L^{\infty}\left(\mathbf{R}^{N}\right)$. From this, the desired estimate follows.

(ii) First, notice that a norm defined by

$$
\|u\|^{2}:=(u, u), \quad(u, v):=\langle u, v\rangle_{\alpha}-\left(1-\delta_{0}\right)\langle u, v\rangle_{L^{2}} \quad \text { for } u, v \in H^{\alpha}\left(\mathbf{R}^{N}\right)
$$

is equivalent to $\|\cdot\|_{\alpha}$ since $0<\delta_{0}<1$. Hence, (62) has a unique solution $v \in H^{\alpha}\left(\mathbf{R}^{N}\right)$ for any $g \in L^{2}\left(\mathbf{R}^{N}\right)$ due to the Lax-Milgram Theorem and it is expressed as $v=K * g$. Since (62) is rewritten as $(1-\Delta)^{\alpha} v=\left(1-\delta_{0}\right) v+g$, we obtain

$$
v=G_{2 \alpha} *\left(\left(1-\delta_{0}\right) v+g\right) .
$$

Thus if $g \in L^{\infty}\left(\mathbf{R}^{N}\right) \cap L^{2}\left(\mathbf{R}^{N}\right)$, using the bootstrap argument and $\mathscr{L}_{2 \alpha}^{p} \subset W^{2 \alpha, p}\left(\mathbf{R}^{N}\right)$, one can check $v \in$ $C_{\mathrm{b}}^{2 \beta}\left(\mathbf{R}^{N}\right)$ for all $\beta \in(0,2 \alpha)$. 
Let us assume $g(x) \geq 0, \not \equiv 0$ and $g \in L^{\infty}\left(\mathbf{R}^{N}\right)$. Since $v=G_{2 \alpha} *\left(\left(1-\delta_{0}\right) v+g\right), G_{2 \alpha}>0$ and $\left(G_{2 \alpha} * g\right)>0$, we observe that

$$
v(x)=\int_{\mathbf{R}^{N}} G_{2 \alpha}(y)\left\{\left(1-\delta_{0}\right) v(x-y)+g(x-y)\right\} \mathrm{d} y>\left(1-\delta_{0}\right) \int_{\mathbf{R}^{N}} G_{2 \alpha}(y) v(x-y) \mathrm{d} y .
$$

Noting $v \in C_{\mathrm{b}}^{\beta}\left(\mathbf{R}^{N}\right)$ and $v(x) \rightarrow 0$ as $|x| \rightarrow \infty$, if $v\left(x_{0}\right)=\min _{\mathbf{R}^{N}} v$ holds for some $x_{0} \in \mathbf{R}^{N}$, then it follows from $\left\|G_{2 \alpha}\right\|_{L^{1}}=1$ that

$$
v\left(x_{0}\right)>\left(1-\delta_{0}\right) \int_{\mathbf{R}^{N}} G_{2 \alpha}(y) v\left(x_{0}-y\right) \mathrm{d} y \geq\left(1-\delta_{0}\right) \int_{\mathbf{R}^{N}} G_{2 \alpha}(y) v\left(x_{0}\right) \mathrm{d} y=\left(1-\delta_{0}\right) v\left(x_{0}\right) .
$$

By $0<\delta_{0}<1$, we get $\min _{\mathbf{R}^{N}} v=v\left(x_{0}\right)>0$, however, this contradicts $v(x) \rightarrow 0$ as $|x| \rightarrow \infty$. Hence, $v$ does not have any global minimum on $\mathbf{R}^{N}$ and this asserts $v(x)>0$ for each $x \in \mathbf{R}^{N}$.

Finally, when $g(x)$ has the compact support, since $v=K * g$ and $K$ decays faster than any polynomial thanks to (i), it is easily seen that $v(x)$ also decays faster than any polynomial.

(iii) Set $w:=v_{2}-v_{1}$. We observe that $w$ satisfies

$$
(1-\Delta)^{\alpha} w-\left(1-\delta_{0}\right) w=g_{2}-g_{1} \geq 0 \quad \text { in } \mathbf{R}^{N} .
$$

Using (ii), one has $w \geq 0$ in $\mathbf{R}^{N}$ and $v_{2}(x) \geq v_{1}(x)$ in $\mathbf{R}^{N}$.

Next, in order to prove Proposition 3.5 and (34), we consider the extension problem observed in [21] (cf. [10]). For $X=(x, t) \in \mathbf{R}_{+}^{N+1}:=\mathbf{R}^{N} \times(0, \infty)$ and $u \in H^{\alpha}\left(\mathbf{R}^{N}\right)$, consider

$$
\left\{\begin{aligned}
t^{1-2 \alpha}\left(-\Delta_{x}+1\right) w-\left(t^{1-2 \alpha} w_{t}\right)_{t}=0 & \text { in } \mathbf{R}_{+}^{N+1} \\
w=u & \text { on } \mathbf{R}^{N}
\end{aligned}\right.
$$

where $\Delta_{x}=\sum_{i=1}^{N} \partial_{x_{i}}^{2}$. We set

$$
X^{\alpha}:=\left\{w(x, t): \mathbf{R}_{+}^{N+1} \rightarrow \mathbf{R} \mid\|w\|_{X^{\alpha}}<\infty\right\}, \quad\|w\|_{X^{\alpha}}^{2}:=\int_{\mathbf{R}_{+}^{N+1}} t^{1-2 \alpha}\left(|\nabla w|^{2}+w^{2}\right) \mathrm{d} X
$$

where $\nabla=\left(\nabla_{x}, \partial_{t}\right)$. First we collect some facts. See, for instance, [19,21].

Proposition A.2. (i) There exists the trace operator $\operatorname{Tr}: X^{\alpha} \rightarrow H^{\alpha}\left(\mathbf{R}^{N}\right)$.

(ii) For any $u \in H^{\alpha}\left(\mathbf{R}^{N}\right)$, (63) has a unique solution $w=E u \in X^{\alpha}$. Furthermore, there exists a $\kappa_{\alpha}>0$ such that Eu satisfies

$$
\int_{\mathbf{R}_{+}^{N+1}} t^{1-2 \alpha}(\nabla E u \cdot \nabla \varphi+E u \varphi) \mathrm{d} X=\kappa_{\alpha}\langle u, \operatorname{Tr} \varphi\rangle_{\alpha}
$$

for all $u \in H^{\alpha}\left(\mathbf{R}^{N}\right)$ and $\varphi \in X^{\alpha}$.

(iii) For every $u \in H^{\alpha}\left(\mathbf{R}^{N}\right)$ and $w \in X^{\alpha}$ with $\operatorname{Tr} w=u$, one has

$$
\kappa_{\alpha}\|u\|_{\alpha}^{2}=\|E u\|_{X^{\alpha}}^{2} \leq\|w\|_{X^{\alpha}}^{2} .
$$

(iv) If $u \in H^{\alpha}\left(\mathbf{R}^{N}\right)$ with $u \geq 0$, then $E u \geq 0$ in $\mathbf{R}_{+}^{N+1}$.

Using these properties, we first show (34), namely,

Lemma A.3. For any $u \in H^{\alpha}\left(\mathbf{R}^{N}\right)$, $\|\mid u\|_{\alpha} \leq\|u\|_{\alpha}$. Moreover, the map $u \mapsto|u|: H^{\alpha}\left(\mathbf{R}^{N}\right) \rightarrow H^{\alpha}\left(\mathbf{R}^{N}\right)$ is continuous.

Proof. Let $u \in H^{\alpha}\left(\mathbf{R}^{N}\right)$. Then it is easily seen that $\|\mid E u\|_{\alpha}=\|E u\|_{\alpha}<\infty$, hence, $|E u| \in X^{\alpha}$. We can also check that $\operatorname{Tr}|E u|=|u|$. Thus, by Proposition A.2 (iii), we have $\kappa_{\alpha}\||u|\|_{\alpha}^{2} \leq\||E u|\|_{X^{\alpha}}^{2}=\|E u\|_{X^{\alpha}}^{2}=\kappa_{\alpha}\|u\|_{\alpha}^{2}$. For the continuity of the map $u \mapsto|u|$, let $u_{n} \rightarrow u_{0}$ in $H^{\alpha}\left(\mathbf{R}^{N}\right)$. From $E u_{n} \rightarrow E u_{0}$ in $X^{\alpha}$ due to Proposition A.2 (iii), we have $\left|E u_{n}\right| \rightarrow\left|E u_{0}\right|$ in $X^{\alpha}$. By $\operatorname{Tr}\left|E u_{n}\right|=\left|u_{n}\right|$ and the boundedness of $\operatorname{Tr}$, we have $\left|u_{n}\right|=\operatorname{Tr}\left|E u_{n}\right| \rightarrow$ $\operatorname{Tr}\left|E u_{0}\right|=\left|u_{0}\right|$ in $H^{\alpha}\left(\mathbf{R}^{N}\right)$.

Now we prove Proposition 3.5.

Proof of Proposition 3.5. The argument is similar to the case $\alpha=1$ (see [9]). For $k \in \mathbf{N}$, set

$$
a_{k}(x):=k \quad \text { if } a(x) \geq k, \quad:=a(x) \quad \text { if }|a(x)|<k, \quad:=-k \quad \text { if } a(x) \leq-k .
$$

Then thanks to (36) and $A \in L^{N /(2 \alpha)}\left(\mathbf{R}^{N}\right)$, we have

$$
\left|a(x)-a_{k}(x)\right| \leq C_{0} A(x) \quad \text { for each }(x, k) \in \mathbf{R}^{N} \times\left[C_{0}, \infty\right), \quad\left\|a-a_{k}\right\|_{L^{N /(2 \alpha)}\left(\mathbf{R}^{N}\right)} \rightarrow 0 .
$$


The first step is to show:

Step 1: For any $\varepsilon>0$ there exists a $\lambda_{\varepsilon}>0$ such that

$$
\int_{\mathbf{R}^{N}}|a| v^{2} \mathrm{~d} x+\int_{\mathbf{R}^{N}}\left|a_{k}\right| v^{2} \mathrm{~d} x \leq \varepsilon[v]_{H^{\alpha}}^{2}+\lambda_{\varepsilon}\|v\|_{L^{2}}^{2} \quad \text { for all } v \in H^{\alpha}\left(\mathbf{R}^{N}\right), k \geq 1
$$

where $[u]_{H^{\alpha}}^{2}:=\int_{\mathbf{R}^{N} \times \mathbf{R}^{N}}|u(x)-u(y)|^{2} /|x-y|^{N+2 \alpha} \mathrm{d} x \mathrm{~d} y$.

From (36) and the definition of $a_{k}$, it follows that $|a(x)|+\left|a_{k}(x)\right| \leq C_{0}(1+A(x))$. Therefore, it suffices to prove

$$
\int_{\mathbf{R}^{N}} A v^{2} \mathrm{~d} x \leq \varepsilon[v]_{H^{\alpha}}^{2}+\lambda_{\varepsilon}\|v\|_{L^{2}}^{2} .
$$

We first notice that for $n \geq 1$,

$$
\int_{\mathbf{R}^{N}} A(x) v^{2} \mathrm{~d} x=\int_{[A<n]} A(x) v^{2} \mathrm{~d} x+\int_{[A \geq n]} A(x) v^{2} \mathrm{~d} x \leq n\|v\|_{L^{2}}^{2}+\int_{[A \geq n]} A(x) v^{2} \mathrm{~d} x .
$$

Using Hölder's inequality and Sobolev's inequality for the second term, we obtain

$$
\int_{[A \geq n]} A(x) v^{2} \mathrm{~d} x \leq\|A\|_{L^{N /(2 \alpha)}([A \geq n])}\|v\|_{L^{2_{\alpha}^{*}}}^{2} \leq C_{S}\|A\|_{L^{N /(2 \alpha)}([A \geq n])}[v]_{H^{\alpha}}^{2} .
$$

Thus

$$
\int_{\mathbf{R}^{N}} A(x) v^{2} \mathrm{~d} x \leq n\|v\|_{L^{2}}^{2}+C_{S}\|A\|_{L^{N /(2 \alpha)}([A \geq n])}[v]_{H^{\alpha}}^{2} .
$$

Since $A \in L^{N /(2 \alpha)}\left(\mathbf{R}^{N}\right)$, choosing $n$ large enough, we get (65) and Step 1 holds.

Step 2: The operators $(1-\Delta)^{\alpha}-a(x)+\lambda_{\varepsilon}$ and $(1-\Delta)^{\alpha}-a_{k}(x)+\lambda_{\varepsilon}$ are coercive on $H^{\alpha}\left(\mathbf{R}^{N}\right)$ for all sufficiently small $\varepsilon>0$.

By Step 1, for sufficiently small $\varepsilon>0$, one sees that

$$
\begin{aligned}
& \int_{\mathbf{R}^{N}}\left(1+4 \pi^{2}|\xi|^{2}\right)^{\alpha}|\widehat{v}(\xi)|^{2} \mathrm{~d} \xi-\int_{\mathbf{R}^{N}} a(x) v^{2} \mathrm{~d} x+\lambda_{\varepsilon}\|v\|_{L^{2}}^{2} \\
\geq & \int_{\mathbf{R}^{N}}\left(1+4 \pi^{2}|\xi|^{2}\right)^{\alpha}|\hat{v}(\xi)|^{2} \mathrm{~d} \xi-\varepsilon[v]_{H^{\alpha}}^{2} \geq \frac{1}{2} \int_{\mathbf{R}^{N}}\left(1+4 \pi^{2}|\xi|^{2}\right)^{\alpha}|\widehat{v}(\xi)|^{2} \mathrm{~d} \xi
\end{aligned}
$$

and

$$
\int_{\mathbf{R}^{N}}\left(1+4 \pi^{2}|\xi|^{2}\right)^{\alpha}|\widehat{v}(\xi)|^{2} \mathrm{~d} \xi-\int_{\mathbf{R}^{N}} a_{k}(x) v^{2} \mathrm{~d} x+\lambda_{\varepsilon}\|v\|_{L^{2}}^{2} \geq \frac{1}{2} \int_{\mathbf{R}^{N}}\left(4 \pi^{2}|\xi|^{2}+m^{2}\right)^{\alpha}|\widehat{v}(\xi)|^{2} \mathrm{~d} \xi .
$$

Hence, Step 2 holds.

Rewrite (35) as follows:

$$
(1-\Delta)^{\alpha} u-a(x) u+\lambda_{\varepsilon} u=\lambda_{\varepsilon} u \quad \text { in } \mathbf{R}^{N} .
$$

Noting Step 2, we may find a unique solution $\psi_{k} \in H^{\alpha}\left(\mathbf{R}^{N}\right)$ of

$$
(1-\Delta)^{\alpha} \psi_{k}-a_{k}(x) \psi_{k}+\lambda_{\varepsilon} \psi_{k}=\lambda_{\varepsilon} u \quad \text { in } \mathbf{R}^{N}
$$

for sufficiently small $\varepsilon>0$. From (67), one observes that $\left(\psi_{k}\right)$ is bounded in $H^{\alpha}\left(\mathbf{R}^{N}\right)$. Furthermore, since $u$ is a unique solution of (68) thanks to (66), we also see from (64) that

$$
\psi_{k} \rightarrow u \quad \text { strongly in } H^{\alpha}\left(\mathbf{R}^{N}\right) .
$$

Next, let $w_{k} \in X^{\alpha}$ be a unique solution of (63) with $u=\psi_{k}$. For $n \in \mathbf{N}$, set

$$
\psi_{k, n}(x):=\left\{\begin{array}{ll}
n & \text { if } \psi_{k}(x) \geq n, \\
\psi_{k}(x) & \text { if }\left|\psi_{k}(x)\right|<n, \\
-n & \text { if } \psi_{k}(x) \leq-n,
\end{array} \quad w_{k, n}(X):= \begin{cases}n & \text { if } w_{k}(X) \geq n, \\
w_{k}(X) & \text { if }\left|w_{k}(X)\right|<n, \\
-n & \text { if } w_{k}(X) \leq-n,\end{cases}\right.
$$

Remark that for every $p \geq 2$,

$$
\begin{aligned}
& \left|w_{k, n}\right|^{p-2} w_{k, n} \in X^{\alpha} \cap L^{\infty}\left(\mathbf{R}_{+}^{N+1}\right), \quad\left|\psi_{k, n}\right|^{p-2} \psi_{k, n} \in H^{\alpha}\left(\mathbf{R}^{N}\right) \cap L^{\infty}\left(\mathbf{R}^{N}\right), \\
& \operatorname{Tr}\left(\left|w_{k, n}\right|^{p-2} w_{k, n}\right)=\left|\psi_{k, n}\right|^{p-2} \psi_{k, n}, \quad\left\|w_{k, n}-w_{k}\right\|_{X^{\alpha}} \rightarrow 0, \quad\left\|\psi_{k, n}-\psi_{k}\right\|_{\alpha} \rightarrow 0 .
\end{aligned}
$$


Step 3: Assume that $u, \psi_{k} \in L^{p}\left(\mathbf{R}^{N}\right)$ and $\psi_{k} \rightarrow u$ strongly in $L^{p}\left(\mathbf{R}^{N}\right)$ for some $p>2$. Then

$$
|u|^{p / 2} \in H^{\alpha}\left(\mathbf{R}^{N}\right), \quad \kappa_{\alpha}\left\||u|^{p / 2}\right\|_{H^{\alpha}}^{2} \leq\left\||w|^{p / 2}\right\|_{X^{\alpha}}^{2} \leq C_{1}\|u\|_{L^{p}}^{p} \quad \text { where } w=E u \in X^{\alpha} .
$$

We use $\left|w_{k, n}\right|^{p-2} w_{k, n}$ as a test function to (63) with $w=w_{k}$ to get

$$
\begin{aligned}
& \int_{\mathbf{R}_{+}^{N+1}} t^{1-2 \alpha}\left\{\nabla w_{k} \cdot \nabla\left(\left|w_{k, n}\right|^{p-2} w_{k, n}\right)+w_{k}\left|w_{k, n}\right|^{p-2} w_{k, n}\right\} \mathrm{d} X \\
= & \kappa_{\alpha} \int_{\mathbf{R}^{N}}\left(a_{k} \psi_{k}-\lambda_{\varepsilon} \psi_{k}+\lambda_{\varepsilon} u\right)\left|\psi_{k, n}\right|^{p-2} \psi_{k, n} \mathrm{~d} x .
\end{aligned}
$$

Notice that

$$
\nabla w_{k} \cdot \nabla\left(\left|w_{k, n}\right|^{p-2} w_{k, n}\right)=(p-1)\left|w_{k, n}\right|^{p-2}\left|\nabla w_{k, n}\right|^{2}=\frac{4}{p^{2}}(p-1)\left|\nabla\left(\left|w_{k, n}\right|^{p / 2}\right)\right|^{2} .
$$

Furthermore, by $\left|w_{k, n}\right| \leq\left|w_{k}\right|,\left|\psi_{k, n}\right| \leq\left|\psi_{k}\right|, w_{k} w_{k, n}=\left|w_{k}\right|\left|w_{k, n}\right|, \psi_{k, n} \psi_{k}=\left|\psi_{k, n}\right|\left|\psi_{k}\right|,\left|a_{k}(x)\right| \leq k$ and $\left|\psi_{k, n}\right|^{p / 2} \in H^{\alpha}\left(\mathbf{R}^{N}\right)$, it follows from Step 1 that

$$
\begin{aligned}
& \int_{\mathbf{R}_{+}^{N+1}} t^{1-2 \alpha} w_{k}\left|w_{k, n}\right|^{p-2} w_{k, n} \mathrm{~d} X \geq \int_{\mathbf{R}_{+}^{N+1}} t^{1-2 \alpha}\left(\left|w_{k, n}\right|^{p / 2}\right)^{2} \mathrm{~d} X \\
& \int_{\mathbf{R}^{N}} \lambda_{\varepsilon} \psi_{k}\left|\psi_{k, n}\right|^{p-2} \psi_{k, n} \mathrm{~d} x \geq \int_{\mathbf{R}^{N}} \lambda_{\varepsilon}\left|\psi_{k, n}\right|^{p} \mathrm{~d} x, \\
& \int_{\mathbf{R}^{N}} a_{k} \psi_{k}\left|\psi_{k, n}\right|^{p-2} \psi_{k, n} \mathrm{~d} x=\left(\int_{\left[\left|\psi_{k}\right|<n\right]}+\int_{\left[\left|\psi_{k}\right| \geq n\right]}\right) a_{k} \psi_{k}\left|\psi_{k, n}\right|^{p-2} \psi_{k, n} \mathrm{~d} x \\
& \leq \int_{\mathbf{R}^{N}}\left|a_{k}\right|\left(\left|\psi_{k, n}\right|^{p / 2}\right)^{2} \mathrm{~d} x+n^{p-1} k \int_{\left[\left|\psi_{k}\right| \geq n\right]}\left|\psi_{k}\right| \mathrm{d} x \\
& \leq \varepsilon\left[\left|\psi_{k, n}\right|^{p / 2}\right]_{H^{\alpha}}^{2}+\lambda_{\varepsilon} \int_{\mathbf{R}^{N}}\left|\psi_{k, n}\right|^{p} \mathrm{~d} x+k n^{p-1} \int_{\left[\left|\psi_{k}\right| \geq n\right]}\left|\psi_{k}\right| \mathrm{d} x .
\end{aligned}
$$

Therefore, we obtain

$$
\begin{aligned}
& \int_{\mathbf{R}_{+}^{N+1}} t^{1-2 \alpha}\left\{\frac{4}{p^{2}}(p-1)\left|\nabla\left(\left|w_{k, n}\right|^{p / 2}\right)\right|^{2}+\left(\left|w_{k, n}\right|^{p / 2}\right)^{2}\right\} \mathrm{d} X \\
\leq & \kappa_{\alpha}\left[\varepsilon\left[\left|\psi_{k, n}\right|^{p / 2}\right]_{H^{\alpha}}^{2}+k n^{p-1} \int_{\left[\left|\psi_{k}\right| \geq n\right]}\left|\psi_{k}\right| \mathrm{d} x+\lambda_{\varepsilon} \int_{\mathbf{R}^{N}}|u|\left|\psi_{k, n}\right|^{p-1} \mathrm{~d} x\right] .
\end{aligned}
$$

Since Proposition A.2 asserts

$$
\kappa_{\alpha}\left\|\left|\psi_{k, n}\right|^{p / 2}\right\|_{H^{\alpha}}^{2} \leq\left\|\left|w_{k, n}\right|^{p / 2}\right\|_{X^{\alpha}}^{2}, \quad n^{p-1} \leq\left|\psi_{k}\right|^{p-1} \quad \text { on }\left[\left|\psi_{k}\right| \geq n\right],
$$

choosing

we finally obtain

$$
\varepsilon=\frac{1}{2} \frac{4(p-1)}{p^{2}}
$$

$$
\left\|\left|w_{k, n}\right|^{p / 2}\right\|_{X^{\alpha}}^{2} \leq C_{p, \alpha}\left[k \int_{\left[\left|\psi_{k}\right| \geq n\right]}\left|\psi_{k}\right|^{p} \mathrm{~d} x+\int_{\mathbf{R}^{N}}\left|u \| \psi_{k, n}\right|^{p-1} \mathrm{~d} x\right] .
$$

Now let us consider the case where $u, \psi_{k} \in L^{p}\left(\mathbf{R}^{N}\right)$ and $\psi_{k} \rightarrow u$ strongly in $L^{p}\left(\mathbf{R}^{N}\right)$. By Hölder's inequality and the definition of $\psi_{k, n}$, we have

$$
\int_{\mathbf{R}^{N}}|u|\left|\psi_{k, n}\right|^{p-1} \mathrm{~d} x \leq\|u\|_{L^{p}}\left\|\psi_{k}\right\|_{L^{p}}^{p-1}
$$

and the right hand side in (70) is bounded as $n \rightarrow \infty$. Since $w_{k, n} \rightarrow w_{k}$ strongly in $X^{\alpha}$, we observe that $\left|w_{k, n}\right|^{p / 2} \rightarrow\left|w_{k}\right|^{p / 2}$ weakly in $X^{\alpha}$. Letting $n \rightarrow \infty$ in (70), one has

$$
\left\|\left|w_{k}\right|^{p / 2}\right\|_{X^{\alpha}}^{2} \leq C_{1}\|u\|_{L^{p}}\left\|\psi_{k}\right\|_{L^{p}}^{p-1} .
$$

Since $\operatorname{Tr}\left(\left|w_{k}\right|^{p / 2}\right)=\left|\psi_{k}\right|^{p / 2}$, Proposition A.2 gives

$$
\kappa_{\alpha}\left\|\left|\psi_{k}\right|^{p / 2}\right\|_{H^{\alpha}}^{2} \leq\left\|\left|w_{k}\right|^{p / 2}\right\|_{X^{\alpha}}^{2} \leq C_{1}\|u\|_{L^{p}}\left\|\psi_{k}\right\|_{L^{p}}^{p-1} .
$$


Thus by Sobolev's inequality, we get

$$
\left\|\left|\psi_{k}\right|^{p / 2}\right\|_{L^{2_{\alpha}^{*}}}^{2} \leq C_{0}\left\|\left|\psi_{k}\right|^{p / 2}\right\|_{H^{\alpha}}^{2} \leq C\|u\|_{L^{p}}\left\|\psi_{k}\right\|_{L^{p}}^{p-1} .
$$

Recalling $\psi_{k} \rightarrow u$ strongly in $L^{p}\left(\mathbf{R}^{N}\right)$ and letting $k \rightarrow \infty$ in (71), we observe that $\left(\left|w_{k}\right|^{p / 2}\right)$ is bounded in $X^{\alpha}$, $\left|w_{k}\right|^{p / 2} \rightarrow|w|^{p / 2}$ weakly in $X^{\alpha}$ and

$$
\left\||w|^{p / 2}\right\|_{X^{\alpha}}^{2} \leq C_{1}\|u\|_{L^{p}}^{p}
$$

where $w=E u \in X^{\alpha}$. Noting $\operatorname{Tr}\left(|w|^{p / 2}\right)=|u|^{p / 2}$, we have

$$
|u|^{p / 2} \in H^{\alpha}\left(\mathbf{R}^{N}\right), \quad \kappa_{\alpha}\left\|\left.u\right|^{p / 2}\right\|_{H^{\alpha}}^{2} \leq\left\||w|^{p / 2}\right\|_{X^{\alpha}}^{2} \leq C_{1}\|u\|_{L^{p}}^{p}
$$

and Step 3 holds.

\section{Step 4: Conclusion}

By Step 3 and (72), if $u, \psi_{k} \in L^{p}\left(\mathbf{R}^{N}\right)$ and $\psi_{k} \rightarrow u$ strongly in $L^{p}\left(\mathbf{R}^{N}\right)$, then

$$
\left\||u|^{p / 2}\right\|_{L^{2_{\alpha}^{*}}}^{2} \leq C\left\||u|^{p / 2}\right\|_{H^{\alpha}}^{2} \leq C \kappa_{\alpha}^{-1}\left\||w|^{p / 2}\right\|_{X^{\alpha}}^{2} \leq C\|u\|_{L^{p}}^{p}, \quad\left\|\left|\psi_{k}\right|^{p / 2}\right\|_{L^{2_{\alpha}^{*}}}^{2} \leq C\|u\|_{L^{p}}\left\|\psi_{k}\right\|_{L^{p}}^{p-1} .
$$

Now we select $p=p_{1}:=2_{\alpha}^{*}>2$. From (69), the assumptions of Step 3 are satisfied and

$$
\left\||u|^{p_{1} / 2}\right\|_{L^{2_{\alpha}^{*}}}^{2} \leq C\left\||u|^{p_{1} / 2}\right\|_{H^{\alpha}}^{2} \leq C \kappa_{\alpha}^{-1}\left\||w|^{p_{1} / 2}\right\|_{X^{\alpha}}^{2} \leq C\|u\|_{L^{p_{1}}}^{p_{1}}, \quad\left\|\left|\psi_{k}\right|^{p_{1} / 2}\right\|_{L^{2_{\alpha}^{*}}}^{2} \leq C\|u\|_{L^{p_{1}}}\left\|\psi_{k}\right\|_{L^{p_{1}}}^{p_{1^{\prime}}-1} .
$$

From this, one observes that the assumptions of Step 3 holds for any $2 \leq p<p_{1} 2_{\alpha}^{*} / 2$. Hence, setting $p_{2}:=p_{1} 2_{\alpha}^{*} / 2$, (73) holds for each $p<p_{2}$. Again, the assumptions of Step 3 hold for each $p<p_{3}:=p_{2} 2_{\alpha}^{*} / 2$. Repeating this argument and noting $2_{\alpha}^{*} / 2>1$, we observe that (73) holds for any $p<\infty$, which implies $u \in L^{p}\left(\mathbf{R}^{N}\right)$ for any $2 \leq p<\infty$. This completes the proof.

Acknowledgement. The author would like to thank Professor Tatsuki Kawakami, Professor Tohru Ozawa and Professor Kazunaga Tanaka for valuable comments and fruitful discussions on the topic of this paper.

\section{REFERENCES}

[1] F.J. Almgren, JR., and E.H. Lieb, Symmetric decreasing rearrangement is sometimes continuous. J. Amer. Math. Soc. 2 (1989), no. $4,683-773.13$

[2] V. Ambrosio, Periodic solutions for the non-local operator $\left(-\Delta+m^{2}\right)^{s}-m^{2 s}$ with $m \geq 0$. arXiv:1510.05808 [math.AP] 1

[3] V. Ambrosio, Periodic solutions for a superlinear fractional problem without the Ambrosetti-Rabinowitz condition. arXiv:1601.06282 [math.AP] 1

[4] V. Ambrosio, Ground states solutions for a non-linear equation involving a pseudo-relativistic Schrödinger operator. arXiv:1601.06827 [math.AP] 1, 3, 4

[5] A. Azzollini and A. Pomponio, On the Schrödinger equation in $\mathbb{R}^{N}$ under the effect of a general nonlinear term. Indiana Univ. Math. J. 58 (2009), no. 3, 1361-1378. 3, 18

[6] H. Berestycki,T. Gallouët, O. Kavian, Équations de champs scalaires euclidiens non linéaires dans le plan. C. R. Acad. Sci. Paris Sér. I Math. 297 (1983), no. 5, 307-310. 2

[7] H. Berestycki and P.-L. Lions, Nonlinear scalar field equations. I. Existence of a ground state. Arch. Rational Mech. Anal. 82 (1983), no. 4, 313-345. 2, 6, 7, 10

[8] H. Berestycki and P.-L. Lions, Nonlinear scalar field equations. II. Existence of infinitely many solutions. Arch. Rational Mech. Anal. 82 (1983), no. 4, 347-375. 2, 6, 7

[9] H. Brézis and T. Kato, Remarks on the Schrödinger operator with singular complex potentials. J. Math. Pures Appl. (9) 58 (1979), no. 2, 137-151. 13, 24

[10] L. Caffarelli and L. Silvestre, An extension problem related to the fractional Laplacian. Comm. Partial Differential Equations 32 (2007), no. 7-9, 1245-1260. 24

[11] X. Chang and Z.-Q. Wang, Ground state of scalar field equations involving a fractional Laplacian with general nonlinearity. Nonlinearity 26 (2013), no. 2, 479-494. 3

[12] W. Choi and J. Seok, Nonrelativistic limit of standing waves for pseudo-relativistic nonlinear Schrödinger equations. J. Math. Phys. 57 (2016), no. 2, 021510, 15 pp. 1, 3

[13] S. Cingolani and S. Secchi, Simone Ground states for the pseudo-relativistic Hartree equation with external potential. Proc. Roy. Soc. Edinburgh Sect. A 145 (2015), no. 1, 73-90. 1

[14] S. Cingolani and S. Secchi, Semiclassical analysis for pseudo-relativistic Hartree equations. J. Differential Equations 258 (2015), no. $12,4156-4179.1$

[15] V. Coti Zelati and M. Nolasco, Existence of ground states for nonlinear, pseudo-relativistic Schrödinger equations. Atti Accad. Naz. Lincei Cl. Sci. Fis. Mat. Natur. Rend. Lincei (9) Mat. Appl. 22 (2011), no. 1, 51-72. 1

[16] V. Coti Zelati and M. Nolasco, Ground states for pseudo-relativistic Hartree equations of critical type. Rev. Mat. Iberoam. 29 (2013), no. 4, 1421-1436. 1

[17] V. Coti Zelati and M. Nolasco, Ground states for pseudo-relativistic equations with combined power and Hartree-type nonlinearities. Recent trends in nonlinear partial differential equations. II. Stationary problems, 151-167, Contemp. Math., 595, Amer. Math. Soc., Providence, RI, 2013. 1 
[18] V. Coti Zelati and P.H. Rabinowitz, Homoclinic type solutions for a semilinear elliptic PDE on $\mathbf{R}^{n}$. Comm. Pure Appl. Math. 45 (1992), no. 10, 1217-1269. 19

[19] F. Demengel and G. Demengel, Functional spaces for the theory of elliptic partial differential equations. Translated from the 2007 French original by Reinie Erné. Universitext. Springer, London; EDP Sciences, Les Ulis, 2012.24

[20] E. Di Nezza, G. Palatucci and E. Valdinoci, Hitchhiker's guide to the fractional Sobolev spaces. Bull. Sci. Math. 136 (2012), no. 5, 521-573. 19

[21] M.M. Fall and V. Felli, Unique continuation properties for relativistic Schrödinger operators with a singular potential. Discrete Contin. Dyn. Syst. 35 (2015), no. 12, 5827-5867. 1, 13, 15, 17, 24

[22] P. Felmer, A. Quaas and J. Tan, Positive solutions of the nonlinear Schrödinger equation with the fractional Laplacian. Proc. Roy. Soc. Edinburgh Sect. A 142 (2012), no. 6, 1237-1262. 19

[23] P. Felmer and I. Vergara, Scalar field equation with non-local diffusion. NoDEA Nonlinear Differential Equations Appl. Online First (2015). 1, 2, 3, 13, 14

[24] J. Hirata, N. Ikoma and K. Tanaka, Nonlinear scalar field equations in $\mathbb{R}^{N}$ : mountain pass and symmetric mountain pass approaches. Topol. Methods Nonlinear Anal. 35 (2010), no. 2, 253-276. 2, 4, 5, 7, 8, 12

[25] L. Jeanjean, Existence of solutions with prescribed norm for semilinear elliptic equations. Nonlinear Anal. 28 (1997), no. 10, 1633-1659. 4, 7

[26] L. Jeanjean and K. Tanaka, A remark on least energy solutions in $\mathbf{R}^{N}$. Proc. Amer. Math. Soc. 131 (2003), no. 8, $2399-2408$. 2

[27] L. Jeanjean and K. Tanaka, Singularly perturbed elliptic problems with superlinear or asymptotically linear nonlinearities. Calc. Var. Partial Differential Equations 21 (2004), no. 3, 287-318. 4, 20

[28] L. Jeanjean and K. Tanaka, A positive solution for a nonlinear Schrödinger equation on $\mathbb{R}^{N}$. Indiana Univ. Math. J. 54 (2005), no. 2, 443-464. 3, 18

[29] P.-L. Lions, Symétrie et compacité dans les espaces de Sobolev. J. Funct. Anal. 49 (1982), no. 3, 315-334. 4, 5

[30] P.-L. Lions, The concentration-compactness principle in the calculus of variations. The locally compact case. I. Ann. Inst. H. Poincaré Anal. Non Linéaire 1 (1984), no. 2, 109-145. 4

[31] P.-L. Lions, The concentration-compactness principle in the calculus of variations. The locally compact case. II. Ann. Inst. H. Poincaré Anal. Non Linéaire 1 (1984), no. 4, 223-283. 4, 19

[32] M. Melgaard and F. Zongo, Multiple solutions of the quasirelativistic Choquard equation. J. Math. Phys. 53 (2012), no. 3, 033709, 12 pp. 1

[33] P.H. Rabinowitz, Minimax methods in critical point theory with applications to differential equations. CBMS Regional Conference Series in Mathematics, 65, the American Mathematical Society, Providence, RI, 1986. 6, 8, 18

[34] S. Secchi, On some nonlinear fractional equations involving the Bessel operator. to appear in J. Dynam. Differential Equations. $1,2,3,4,13,14$

[35] E.M. Stein, Singular integrals and differentiability properties of functions. Princeton Mathematical Series, No. 30 Princeton University Press, Princeton, N.J. 1970. 4, 14

[36] E.M. Stein, Harmonic analysis: real-variable methods, orthogonality, and oscillatory integrals. Princeton Mathematical Series, vol. 43, Princeton University Press, Princeton, NJ, 1993. With the assistance of Timothy S. Murphy; Monographs in Harmonic Analysis, III. 23

[37] W.A. Strauss, Existence of solitary waves in higher dimensions. Comm. Math. Phys. 55 (1977), no. 2, 149-162. 10

[38] J. Tan, Y. Wang and J. Yang, Nonlinear fractional field equations. Nonlinear Anal. 75 (2012), no. 4, 2098-2110. 1, 3, 19

[39] M. Willem, Minimax theorems. Progress in Nonlinear Differential Equations and their Applications, 24. Birkhäuser Boston, Inc., Boston, MA, 1996. 5

Faculty of Mathematics and Physics, Institute of Science and Engineering, Kanazawa University, Kakuma, Kanazawa, ISHIKAWA 9201192, JAPAN

E-mail address: ikoma@se.kanazawa-u.ac.jp 